\title{
Main Problems of the Theoretical Physics and Artifacts of Local Physics
}

\author{
Boris V. Alexeev \\ Russian State Technological University, Moscow, Russia \\ Email: Boris.Vlad.Alexeev@gmail.com
}

How to cite this paper: Alexeev, B.V. (2021) Main Problems of the Theoretical Physics and Artifacts of Local Physics. Journal of Modern Physics, 12, 1048-1108. https://doi.org/10.4236/jmp.2021.127066

Received: April 19, 2021

Accepted: May 28, 2021

Published: May 31, 2021

Copyright $\odot 2021$ by author(s) and Scientific Research Publishing Inc. This work is licensed under the Creative Commons Attribution International License (CC BY 4.0).

http://creativecommons.org/licenses/by/4.0/

\begin{abstract}
Shortcomings of the Boltzmann physical kinetics are considered. Boltzmann equation is only plausible equation. The cosequences originated from this fact are considered in the different fields of theoretical physics from the point of view of nonlocal physics. Namely: main principles of nonlocal physics; generalized hydrodynamic equations; magnetic field evolution in the superconductor of the second type; Hubble expansion; special theory of relativity; the problem of the interaction of matter (M) with physical vacuum (PV) is considered including the $\mathrm{PV}-\mathrm{M}$ energy exchange. Application nonlocal physics to the problem of the dark matter existence-dark matter does not exist, analytical investigation.
\end{abstract}

\section{Keywords}

Nonlocal Physics, Interaction of Matter With Physical Vacuum, Antimatter Evolution After Big Bang, Dark Matter Problem, Transport Processes in Physical Vacuum

\section{Introduction}

By the end of the twentieth century it was found that $96 \%$ of matter and energy in the Universe is of unknown origin. The terminology "dark matter" and "dark" energy was introduced in the scientific language. But even this would not be such a depressing fact, if it were not for the belief that this dark matter and dark energy are not diagnosed, and only the indirect effects on space objects can judge the existence of dark matter and dark energy. We even had to face the opinion of religious-minded people who claimed that four percent is all that the Creator left for the study to man. In practice, science has faced the most serious challenge since the fundamental monograph of I. Newton "Mathematical principles of natural philosophy" was published in 1687. On the Internet you can 
find a reaction to the current situation, bordering on despair. I initially assumed that the nonlocal physics developed by me would lead to very significant changes in the description of natural phenomena. That is to say, $4 \%$ of new information compared to $96 \%$ of the classical theory, but not Vice versa! What effect as a result! It is impossible to believe that more than three hundred years after Newton, world science came to such a disappointing result. Then the question ariseswhere is the error? The answer to this question is given by nonlocal physics. In other words, the origin of all difficulties lies in the total oversimplification, which follows from the fundamental shortcomings of the local statistical theory of transport processes.

In fact, the crisis has evolved over the years. The first heavy blow in this way the unresolved physical problems, was the suicide of the great physicist $\mathrm{L}$. Boltzmann; according to the belief of many (including Acad. M. A. Leontovich) connected with unsolved problems and criticism of Boltzmann kinetic theory, the first of the following list of unsolved problems of fundamental physics (local physical kinetics of dissipative processes):

1) Kinetic theory of entropy and the problem of "primary" fluctuation.

2) A strict theory of turbulence.

3) Quantum non-relativistic and relativistic hydrodynamics, the theory of charge separation in atomic structures and nuclei. High temperature superconductivity.

4) The theory of ball lightning.

5) The theory of dark matter.

6) The theory of dark energy. Hubble expansion of the Universe.

7) The destiny of antimatter after the Big Bang.

8) Unified theory of dissipative structures, from atomic structure to cosmology.

These problems can not be solved within the framework of local physics, but find their natural solution as a special case of solving the problem 8-the creation of a unified theory of dissipative structures-from atomic structures to cosmology, [1]-[8].

I repeat-the origin of difficulties in theoretical physics consists in the total Oversimplification following from the principles of local physics and reflects the general shortcomings of the local kinetic transport theory based on the Boltzmann kinetic theory.

\section{Boltzmann Equation is Only a Plausible Equation}

The Boltzmann equation [1] works from the molecular to cosmological level, but has an amazing origin and equally obvious drawbacks. Regarding the origin, the equation is based on Newtonian mechanics, which contains in the equation the second derivative by time. But the Boltzmann kinetic equation has only the first temporal derivative. This fact leads to the irreversibility of the processes; hence the irreversible nature of the evolution of $H$-functions and the inevitable ques- 
tion: where does the initial fluctuation appear from, if Boltzmann kinetic theory does not contain fluctuations in principle? You do not even need (initially) to write equations. Indeed, Boltzmann physical kinetics is based on a reduced description of dissipative processes and the principle of local thermodynamic equilibrium (LTE).

It is assumed that the distribution function (DF) is not changed within a physically infinitely small volume (say, a $\mathrm{PhSV}_{1}$ ) that contains, however, enough particles for the introduction of macroscopic parameters (such as temperature and concentration), which are constant within PhSV. But PhSV is an open thermodynamic system that responds to the environment only after its interaction with foreign particles, penetrated from a neighboring PhSV; in other words, after a time $\tau$ of order of the average time between collisions. Then in the simplest case of the gas objects nonlocal parameter $\tau$ can be considered as a corresponding relaxation time.

Transport processes in open dissipative systems are considered in physical kinetics. Therefore, the kinetic description is inevitably related to the system diagnostics. Such an element of diagnostics in the case of theoretical description in physical kinetics is the concept of the physically infinitely small volume (PhSV). The correlation between theoretical description and system diagnostics is well-known in physics. Suffice it to recall the part played by test charge in electrostatics or by test circuit in the physics of magnetic phenomena.

The traditional definition of PhSV contains the statement to the effect that the $\mathrm{PhSV}$ contains a sufficient number of particles for introducing a statistical description; however, at the same time, the PhSV is much smaller than the volume $V$ of the physical system under consideration; in a first approximation, this leads to local approach in investigating the transport processes.

It is assumed in classical hydrodynamics that local thermodynamic equilibrium is first established within the PhSV, and only after that the transition occurs to global thermodynamic equilibrium if it is at all possible for the system under study. Let us consider the hydrodynamic description in more detail from this point of view. Assume that we have two neighboring physically infinitely small volumes $\mathrm{PhSV}_{1}$ and $\mathrm{PhSV}_{2}$ in a non-equilibrium system. The one-particle distribution function (DF) $f_{s m, 1}\left(\mathbf{r}_{1}, \mathbf{v}, t\right)$ corresponds to the volume $\mathrm{PhSV}_{1}$, and the function $f_{s m, 2}\left(\mathbf{r}_{2}, \mathbf{v}, t\right)$-to the volume $\mathrm{PhSV}_{2}$. It is assumed in a first approximation that $f_{s m, 1}\left(\mathbf{r}_{1}, \mathbf{v}, t\right)$ does not vary within $\mathrm{PhSV}_{1}$, same as $f_{s m, 2}\left(\mathbf{r}_{2}, \mathbf{v}, t\right)$ does not vary within the neighboring volume $\mathrm{PhSV}_{2}$. It is this assumption of locality that is implicitly contained in the Boltzmann equation (BE) [2]-[8]. However, the assumption is too crude. Indeed, a particle on the boundary between two volumes, which experienced the last collision in $\mathrm{PhSV}_{1}$ and moves toward $\mathrm{PhSV}_{2}$, introduces information about the $f_{s m, 1}\left(\mathbf{r}_{1}, \mathbf{v}, t\right)$ into the neighboring volume $\mathrm{PhSV}_{2}$. Similarly, a particle on the boundary between two volumes, which experienced the last collision in $\mathrm{PhSV}_{2}$ and moves toward $\mathrm{PhSV}_{1}$, introduces information about the DF $f_{s m, 2}\left(\mathbf{r}_{2}, \mathbf{v}, t\right)$ into the neighbor- 
ing volume $\mathrm{PhSV}_{1}$. The relaxation over translational degrees of freedom of particles of like masses occurs during several collisions. As a result, "Knudsen layers" are formed on the boundary between neighboring physically infinitely small volumes, the characteristic dimension of which is of the order of path length. Therefore, a correction must be introduced into the DF in the PhSV, which is proportional to the mean time between collisions and to the substantive derivative of the DF.

Let a particle of finite radius be characterized as before by the position $\mathbf{r}$ at the instant of time $t$ of its center of mass moving at velocity $\mathbf{v}$. Then, the situation is possible where, at some instant of time $t$, the particle is located on the interface between two volumes. In so doing, the lead effect is possible (say, for $\mathrm{PhSV}_{2}$ ), when the center of mass of particle moving to the neighboring volume $\mathrm{PhSV}_{2}$ is still in $\mathrm{PhSV}_{1}$. However, the delay effect takes place as well, when the center of mass of particle moving to the neighboring volume (say, $\mathrm{PhSV}_{2}$ ) is already located in $\mathrm{PhSV}_{2}$ but a part of the particle still belongs to $\mathrm{PhSV}_{1}$.

Moreover, even the point-like particles (starting after the last collision near the boundary between two mentioned volumes) can change the distribution functions in the neighboring volume. The adjusting of the particles dynamic characteristics for translational degrees of freedom takes several collisions. As result, we have in the definite sense "the Knudsen layer" between these volumes. This fact unavoidably leads to fluctuations in mass and hence in other hydrodynamic quantities. Existence of such "Knudsen layers" is not connected with the choice of space nets and fully defined by the reduced description for ensemble of particles of finite diameters in the conceptual frame of open physically small volumes, therefore with the chosen method of measurement.

This entire complex of effects defines non-local effects in space and time. The corresponding situation is typical for the theoretical physics-we could remind about the role of probe charge in electrostatics or probe circuit in the physics of magnetic effects.

The physically infinitely small volume (PhSV) is an open thermodynamic system for any division of macroscopic system by a set of PhSVs.

Let us give some explanations on the qualitative level of investigation. Suppose that the distribution function (DF) $f$ corresponds to $\mathrm{PhSV}_{1}$ and DF $f-\Delta f$ is connected with $\mathrm{PhSV}_{2}$ for Boltzmann particles. In the boundary area in the first approximation, fluctuations will be proportional to the mean free path (or, equivalently, to the mean time between the particle collisions). Then for $\mathrm{PhSV}$ the correction for DF should be introduced as

$$
f^{a}=f-\tau D f / D t
$$

in the left hand side of classical KE describing the translation of DF in phase space. As the result

$$
D f^{a} / D t=J^{B},
$$

where $J^{B}$ is the Boltzmann local collision integral. Important to notice that it 
is only qualitative explanation of the generalized $\mathrm{BE}$ derivation obtained earlier (see for example [2]-[8]) by different strict methods from the BBGKY chain of kinetic equations. The structure of the $\mathrm{KE}_{f}$ is generally as follows

$$
\frac{D f}{D t}=J^{B}+J^{n l},
$$

where $J^{n l}$ is the non-local integral term incorporating in particular the time delay effect. The generalized Boltzmann physical kinetics, in essence, involves a local approximation

$$
J^{n l}=\frac{D}{D t}\left(\tau \frac{D f}{D t}\right)
$$

for the second collision integral, here $\tau$ being proportional to the mean time between the particle collisions. All of the known methods of deriving kinetic equation relative to one-particle DF lead to approximation (2.4), including the method of many scales, the method of correlation functions, and the iteration method. We can draw here an analogy with the Bhatnagar-Gross-Krook (BGK) approximation for $J^{B}$,

$$
J^{B}=\frac{f_{0}-f}{\tau},
$$

which popularity as a means to represent the Boltzmann collision integral is due to the huge simplifications it offers. In other words - the local Boltzmann collision integral admits approximation via the BGK algebraic expression, but more complicated non-local integral can be expressed as differential form (2.4). The ratio of the second to the first term on the right-hand side of Equation (2.3) is given to an order of magnitude as $J^{n l} / J^{B} \approx O\left(\mathrm{Kn}^{2}\right)$ and at large Knudsen numbers (defining as ratio of mean free path of particles to the character hydrodynamic length) these terms become of the same order of magnitude. It would seem that at small Knudsen numbers answering to hydrodynamic description the contribution from the second term on the right-hand side of Equation (2.3) is negligible.

This is not the case, however. When one goes over to the hydrodynamic approximation (by multiplying the kinetic equation by collision invariants and then integrating over velocities), the Boltzmann integral part vanishes, and the second term on the right-hand side of Equation (2.3) gives a single-order contribution in the generalized Navier-Stokes description. Mathematically, we cannot neglect a term with a small parameter in front of the higher derivative. Physically, the appearing additional terms are due to viscosity and they correspond to the small-scale Kolmogorov turbulence [2]-[8].

The integral term $J^{n l}$ turns out to be important both at small and large Knudsen numbers in the theory of transport processes.

Thus, $\tau D f / D t$ is the distribution function fluctuation, and writing Equation (2.2) without taking into account Equation (2.1) makes the BE non-closed. From viewpoint of the fluctuation theory, Boltzmann employed the simplest possible 
closure procedure $f^{a}=f$ in (2.2).

Then, the additional GBE terms (as compared to the BE) are significant for any $\mathrm{Kn}$, and the order of magnitude of the difference between the BE and GBE solutions is impossible to tell beforehand. For GBE the generalized $\mathrm{H}$-theorem is proven [2]-[8].

Boltzmann equation (BE) fully ignores non-local effects and contains only the local collision integral $J^{B}$. The foregoing nonlocal effects are insignificant only in equilibrium systems, where the kinetic approach changes to methods of statistical mechanics.

Results [2]-[8]:

1) Kinetic theory must be non-local in principle.

2) The effect is of the order of the Knudsen number; since nonlocal effects are proportional to the Knudsen number; then we have an opportunity of the description of nonlocal effects in the framework of the two scale approximation.

3) The effect is due to reduced description and not associated with a specific division of a physical system by a net of PhSV.

4) Accurate derivation of the kinetic equation $(\mathrm{KE})$ relative to one-particle $\mathrm{DF}$ leads to corrections of the order of the Knudsen number even before the decoupling of the Bogolyubov hierarchy.

5) This means that in the Boltzmann equation the terms of the order of the Knudsen number are lost; these terms of the order of the Knudsen number, important at large and at small Knudsen numbers.

6) The Boltzmann equation does not belong even to the class of minimal models as being the only plausible equation.

7) The Boltzmann equation in this sense is the wrong equation.

It is clear that this is a revolution in the theory of dissipative processes, in particular in hydrodynamics. In the hydrodynamic Navier-Stokes equation, which is a direct consequence of the Boltzmann equation (BE), the terms of the order proportional to a viscosity are partly lost. It leads to the problem of turbulence and the problems of existence and uniqueness of solutions of the Navier-Stokes equations.

The rigorous approach to derivation of kinetic equation relative to one-particle DF $f\left(K E_{f_{1}}\right)$ is based on employing the hierarchy of Bogolyubov equations [2]-[8]. Generalized Boltzmann physical kinetics brings the strict approximation of non-local effects in space and time and after transfer to the local approximation leads to parameter $\tau$. The appearance of the nonlocal $\tau$ parameter is consistent with the Heisenberg uncertainty relation. In the general case, the parameter $\tau$ is the non-locality parameter; in quantum hydrodynamics, its magnitude is correlated with the "time-energy" uncertainty relation [4]. But in principle generalized kinetic nonlocal equation (and therefore generalized hydrodynamic equations (GHE)) needn't in using of the "time-energy" uncertainty relation for estimation of the value of the non-locality parameter $\tau$. Moreover the "time-energy" uncertainty relation does not produce the exact relations and 
from position of non-local physics is only the simplest estimation of the nonlocal effects.

Really, let us consider two neighboring physically infinitely small volumes $\mathrm{PhSV}_{1}$ and $\mathrm{PhSV}_{2}$ in a non-equilibrium system. Obviously the time $\tau$ should tends to diminish with increasing of the velocities $u$ of particles invading in the nearest neighboring physically infinitely small volume $\left(\mathrm{PhSV}_{1}\right.$ or $\left.\mathrm{PhSV}_{2}\right)$ :

$$
\tau=H_{\tau} / u^{n} .
$$

But the value $\tau$ cannot depend on the velocity direction and naturally to tie $\tau$ with the particle kinetic energy, then

$$
\tau=H_{\tau} / m u^{2},
$$

where $H_{\tau}$ is a coefficient of proportionality, which reflects the state of physical system. In the simplest case $H_{\tau}$ is equal to Plank constant $\hbar$ and relation (2.7) became compatible with the Heisenberg relation. The non-locality parameter $\tau$ plays the same role as the transport coefficients in local hydrodynamics. The different models can be introduced for the $\tau$ definition, but the corresponding results not much different like in local kinetic theory for different models of the particles interaction.

It is known that Ehrenfest adiabatic theorem is one of the most important and widely studied theorems in Schrödinger quantum mechanics. It states that if we have a slowly changing Hamiltonian that depends on time, and the system is prepared in one of the instantaneous eigenstates of the Hamiltonian then the state of the system at any time is given by an the instantaneous eigenfunction of the Hamiltonian up to multiplicative phase factors. The adiabatic theory can be naturally incorporated in generalized quantum hydrodynamics based on local approximations of non-local terms. The adiabatic theorem and consequences of this theory deliver the general quantization conditions for non-local quantum hydrodynamics, [4].

\section{Shortcomings of the Schrödinger Quantum Mechanics}

We now turn to the logic of the development of the non-local theory:

A) In 1926 Madelung published a brilliant article [9] in which he transformed the quantum postulate (Schrödinger equation) in hydrodynamics. In other words, the evolution of a single bound electron was possible to interpret as some effective flow.

B) In 1964 John Stewart Bell [10] found that local statistical theory of dissipative processes is incorrect in principle.

C) In 2007 I found that the Schrödinger equation and hydrodynamic Madelung's form are a deep particular case of nonlocal kinetic equations (see for example [11] [12]) as a result of the transition to the local limit of non-local equations.

This means that generalized physical kinetics (as created earlier by me) has been extended to quantum physics in the form of quantum hydrodynamics. I 
would even say such emotional words-the biggest secret of the Schrödinger equation (SE) is a strange thing-why, in fact, it generally works? Honestly, it starts to work when we go beyond the postulate that it is. Here just note again that:

1) SE is not able to give a self-consistent description of the nucleus - electron shell.

2) SE does not lead to an independent analogue of the hydrodynamic energy equation.

3) SE is not a dissipative equation and therefore cannot be applied to the description of dissipative processes in nanotechnology.

Generalized hydrodynamic equations (GHE) should contain Schrödinger equation (SE) as a deep special case. This affirmation was proved in articles [11] [12]. In other words, we formulated in explicit form all assumptions (all steps) that should be implemented to obtain SE from GHE.

So, we can state that SE is a deep special case of generalized hydrodynamic equations. This means that a new quantum mechanics of dissipative processes has been created.

The Boltzmann equation essentially "does not work" at the distances of the order of the radius of interaction of particles and, therefore, can not be effectively used in the theoretical study of nanotechnology even in the framework of "plausible" models.

It is established that the theory of transport processes (including quantum mechanics) can be presented within the framework of the universal theory (unified theory of dissipative systems) based on nonlocal physical description. It is shown, in particular, that the equations of nonlocal physics lead to the appearance of solitons, which supports the Schrödinger opinion, who interpreted quantum mechanics from the point of view of the existence of waves of matter. The Schrödinger equation is not dissipative. Therefore, the generalized quantum hydrodynamics is a tool for solving problems in the theory of dissipative nano-systems.

\section{Modification of Maxwell Equations}

Notice that the application of the above principles also leads to the modification of the system of Maxwell equations. While the traditional formulation of this system does not involve the continuity equation, its derivation explicitly employs the equation

$$
\frac{\partial \rho^{a}}{\partial t}+\frac{\partial}{\partial \mathbf{r}} \cdot \mathbf{j}^{a}=0
$$

where $\rho^{a}$ is the charge per unit volume, and $\mathbf{j}^{a}$ a the current density, both calculated without accounting for the fluctuations. As a result, the system of Maxwell equations written in the standard notation, namely

$$
\frac{\partial}{\partial \mathbf{r}} \cdot \mathbf{B}=0, \frac{\partial}{\partial \mathbf{r}} \cdot \mathbf{D}=\rho^{a}, \frac{\partial}{\partial \mathbf{r}} \times \mathbf{E}=-\frac{\partial \mathbf{B}}{\partial t}, \frac{\partial}{\partial \mathbf{r}} \times \mathbf{H}=\mathbf{j}^{a}+\frac{\partial \mathbf{D}}{\partial t}
$$

contains 


$$
\rho^{a}=\rho-\rho^{f l}, \quad \mathbf{j}^{a}=\mathbf{j}-\mathbf{j}^{f l} .
$$

The $\rho^{f l}$ and $\mathbf{j}^{f l}$ fluctuations calculated using the GBE are given, for example, in Ref. [7], see also [4]. In rarefied media both effects lead to Johnson's flicker noise observed in 1925 for the first time by J.B. Johnson by the measurement of current fluctuations of thermo-electron emission.

As an example of applying of the generalized Maxwell equations we consider the distribution of the magnetic field in a superconductor Type II from position of nonlocal physics. Usually superconductors are categorized as Type-I or Type-II. Type-I superconductors support only Meissner and normal states, while Type-II superconductors form magnetic vortices in sufficiently strong applied magnetic fields. When the Type I superconductor is placed in the magnetic field, it suddenly or easily looses its superconductivity at critical magnetic field $H_{c}$. After $H_{o}$ the Type I superconductor will become conductor. Example of Type I superconductors: Aluminum $\left(H_{c}=0.0105\right.$ Tesla $)$, Zinc $\left(H_{c}=0.0054\right.$ Tesla).

Type II superconductors are those superconductors which loose their superconductivity gradually but not easily or abruptly when placed in the external magnetic field. Type II superconductors start to loose their superconductivity at lower critical magnetic field $H_{c 1}$ and completely loose their superconductivity at upper critical magnetic field $H_{c 2}$. The state between the lower critical magnetic field $H_{c 1}$ and upper critical magnetic field $H_{c 2}$ is known as vortex state or intermediate state. After magnetic field $H_{c 2}$, the Type II superconductor will become conductor. Except for the elements vanadium, technetium and niobium, the Type II category of superconductors is comprised of metallic compounds and alloys. This new category of superconductors was identified by L.V. Shubnikov [13] at the Kharkov Institute of Science and Technology in the Ukraine in 1936 when he found two distinct critical magnetic fields (known now as $H_{c 1}$ and $H_{c 2}$ ) in $\mathrm{PbTl} 2$. Since a Type II will allow some penetration by an external magnetic field into its surface, this creates some rather novel mesoscopic phenomena like superconducting filaments, stripes or flux-lattice vortices.

The superconducting current flows in a layer with a thickness equal to the penetration depth, which makes it pointless to make large-diameter wires from a superconductor. Actually used for passing through a current-carrying superconducting cable consists of many thin superconducting filaments in a matrix. This design makes it possible to use the superconductor cross-section as efficiently as possible, provides sufficiently high mechanical properties, and provides shunting of superconductor sections in case of loss superconducting properties (for example, when the critical field is accidentally exceeded). The typical superconducting "hair" of such a cable has a diameter of several microns.

The Abrikosov vortex is a vortex of superconducting current (supercurrent) that circulates around a normal (non-superconducting) core (the vortex filament), inducing a magnetic field with a magnetic flux equivalent to the magnetic flux quantum. It was discovered by the physicist A. A. Abrikosov in 1957. In his work "On the magnetic properties of superconductors of the second group" [14], 
it was theoretically shown that the penetration of a magnetic field into a Type II superconductor occurs in the form of quantized vortex filaments. Each such thread (vortex) has a normal (non-superconducting) core with a radius of the order of the superconductor coherence length.

From position of the BCS theory around this normal cylinder, in a region with a radius of the order of the depth of penetration of the magnetic field, a vortical undamped current of Cooper pairs (supercurrent) flows, oriented so that the magnetic field (created by it) is directed along the normal core; that is, coincides with the direction of the external magnetic field. In this case, each vortex carries one quantum of the magnetic flow and

$$
B(r) \sim \sqrt{\frac{\lambda}{r}} \mathrm{e}^{-r / \lambda},
$$

where $\lambda$ is the London depth. But the Abrikosov solution [14] is, strictly speaking, incorrect. In the ideal case, which is considered in his work, there may be a state different from the predicted one. In order to honestly describe the Abrikosov state, we need to take into account fluctuations and consider a superconductor with finite dimensions. This is quite a difficult task which needs applying of the nonlocal physics methods. Abrikosov considered the ideal case and neglected fluctuations. The solution turned out to be correct in the sense that a vortex state is actually observed in real superconductors (see also [15]-[21]).

Let us consider the magnetic field distribution in the "hair' from the position of non-local physics. The filament is considered as the long cylinder which radius is $r_{0}$. Let us use equation (see Equation (10.22) in [22]) written in the form

$$
\left[1+2 \frac{\tau}{\tau_{r}}\right] \frac{\partial^{2}}{\partial t^{2}} \frac{\partial \mathbf{B}}{\partial t}+\frac{1}{\tau_{r}} \frac{\partial}{\partial t} \frac{\partial \mathbf{B}}{\partial t}=\frac{\tau}{\tau_{r}} v_{\phi}^{2} \frac{\partial}{\partial t} \Delta \mathbf{B}-v_{\phi}^{2} \frac{\partial}{\partial t}\left\{\frac{\partial}{\partial \mathbf{r}} \times\left[\frac{\partial}{\partial \mathbf{r}} \times \mathbf{B}\right]\right\},
$$

where $\tau_{r}$ is relaxation time, $v_{\phi}$ is phase velocity. Let us transform (4.5)

$$
\left[1+2 \frac{\tau}{\tau_{r}}\right] \frac{\partial^{2}}{\partial t^{2}} \frac{\partial \mathbf{B}}{\partial t}+\frac{1}{\tau_{r}} \frac{\partial}{\partial t} \frac{\partial \mathbf{B}}{\partial t}=\frac{\tau}{\tau_{r}} v_{\phi}^{2} \frac{\partial}{\partial t} \Delta \mathbf{B}+v_{\phi}^{2} \frac{\partial}{\partial t} \Delta \mathbf{B}
$$

or

$$
\left[1+2 \frac{\tau}{\tau_{r}}\right] \frac{\partial^{2}}{\partial t^{2}} \frac{\partial \mathbf{B}}{\partial t}+\frac{1}{\tau_{r}} \frac{\partial}{\partial t} \frac{\partial \mathbf{B}}{\partial t}=\left[1+\frac{\tau}{\tau_{r}}\right] v_{\phi}^{2} \frac{\partial}{\partial t} \Delta \mathbf{B}
$$

Let us introduce the scales

$$
t \leftrightarrow \tau_{r}, \quad r \leftrightarrow r_{0}=v_{\phi} \tau_{r}, \quad B \leftrightarrow B_{0}
$$

Then

$$
\left[1+2 \frac{\tau}{\tau_{r}}\right] \frac{1}{\tau_{r}^{3}} \frac{\partial^{2}}{\partial \tilde{t}^{2}} \frac{\partial \tilde{\mathbf{B}}}{\partial \tilde{t}}+\frac{1}{\tau_{r}^{3}} \frac{\partial}{\partial \tilde{t}} \frac{\partial \tilde{\mathbf{B}}}{\partial \tilde{t}}=\left[1+\frac{\tau}{\tau_{r}}\right] v_{\phi}^{2} \frac{\partial}{\partial t} \Delta \tilde{\mathbf{B}}
$$

or

$$
\left[1+2 \frac{\tau}{\tau_{r}}\right] \frac{\partial^{2}}{\partial \tilde{t}^{2}} \frac{\partial \tilde{\mathbf{B}}}{\partial \tilde{t}}+\frac{\partial}{\partial \tilde{t}} \frac{\partial \tilde{\mathbf{B}}}{\partial \tilde{t}}=\left[1+\frac{\tau}{\tau_{r}}\right] v_{\phi}^{2} \tau_{r}^{3} \frac{\partial}{\partial t} \Delta \tilde{\mathbf{B}}
$$

or 


$$
\left[1+2 \frac{\tau}{\tau_{r}}\right] \frac{\partial^{2}}{\partial \tilde{t}^{2}} \frac{\partial \tilde{\mathbf{B}}}{\partial \tilde{t}}+\frac{\partial}{\partial \tilde{t}} \frac{\partial \tilde{\mathbf{B}}}{\partial \tilde{t}}=\left[1+\frac{\tau}{\tau_{r}}\right] \tau_{r} \frac{\partial}{\partial t} \tilde{\Delta} \tilde{\mathbf{B}}
$$

or

$$
\left[1+2 \frac{\tau}{\tau_{r}}\right] \frac{\partial^{2}}{\partial \tilde{t}^{2}} \frac{\partial \tilde{\mathbf{B}}}{\partial \tilde{t}}+\frac{\partial}{\partial \tilde{t}} \frac{\partial \tilde{\mathbf{B}}}{\partial \tilde{t}}=\left[1+\frac{\tau}{\tau_{r}}\right] \frac{\partial}{\partial \tilde{t}} \tilde{\Delta} \tilde{\mathbf{B}}
$$

We use spherical coordinate system. It is naturally supposing that in superconducting regime $\mathbf{B}=\mathbf{B}(r)$. Then after integration (4.12) on time we obtain

$$
\left[1+2 \frac{\tau}{\tau_{r}}\right] \frac{\partial^{2} \tilde{B}}{\partial \tilde{t}^{2}}+\frac{\partial \tilde{B}}{\partial \tilde{t}}=\left[1+\frac{\tau}{\tau_{r}}\right] \tilde{\Delta} \tilde{B}+\tilde{f}(\tilde{r}),
$$

where $\tilde{f}(\tilde{r})$ is a function of $\tilde{r}$. The specific type of this function is determined by the task conditions. If $\tau \ll \tau_{r}$ we have

$$
\frac{\partial^{2} \tilde{B}}{\partial \tilde{t}^{2}}+\frac{\partial \tilde{B}}{\partial \tilde{t}}=\tilde{\Delta} \tilde{B}+\tilde{f}(\tilde{r})
$$

For the stationary case

$$
\tilde{\Delta} \tilde{B}+\tilde{f}(\tilde{r})=0
$$

or in the mentioned spherical coordinate system we reach in the case of the radial symmetry

$$
\frac{\partial^{2} \tilde{B}}{\partial \tilde{r}^{2}}+\frac{2}{\tilde{r}} \frac{\partial \tilde{B}}{\partial \tilde{r}}+\tilde{f}(\tilde{r})=0
$$

Let us choose the arbitrary function $\tilde{f}(\tilde{r})$ in the form (4.17). Obviously this form corresponds to the magnetic field distribution around a rectilinear infinite conductor with current $(C=$ const $)$

$$
\tilde{f}(\tilde{r})=C\left[\tilde{B}(\tilde{r})-\frac{1}{\tilde{r}}\right]
$$

we have

$$
\frac{\partial^{2} \tilde{B}}{\partial \tilde{r}^{2}}+\frac{2}{\tilde{r}} \frac{\partial \tilde{B}}{\partial \tilde{r}}+C\left(\tilde{B}-\frac{1}{\tilde{r}}\right)=0
$$

or

$$
\tilde{r} \frac{\partial^{2} \tilde{B}}{\partial \tilde{r}^{2}}+2 \frac{\partial \tilde{B}}{\partial \tilde{r}}+C \tilde{r} \tilde{B}-C=0 .
$$

Equation (4.19) has analytical solution. For example for the case $C=1$ we have equation

$$
\tilde{r} \frac{\partial^{2} \tilde{B}}{\partial \tilde{r}^{2}}+2 \frac{\partial \tilde{B}}{\partial \tilde{r}}+\tilde{r} \tilde{B}-1=0
$$

and its solution in the complex form

$$
\tilde{B}(\tilde{r})=\frac{C_{1} \mathrm{e}^{-i \tilde{r}}}{\tilde{r}}-\frac{i C_{2} \mathrm{e}^{i \tilde{r}}}{2 \tilde{r}}+\frac{1}{\tilde{r}} .
$$

The real part of the solution (4.21) is written as 


$$
\tilde{B}_{\text {real }}(\tilde{r})=\frac{1}{\tilde{r}}\left(C_{1} \cos \tilde{r}+\frac{C_{2}}{2} \sin \tilde{r}+1\right) .
$$

This statement can be verified by directly substituting the solution (4.21) and (4.22) into the equation (4.20). Extremes can be found from

$$
\tilde{B}_{\text {real }}(\tilde{r})=\frac{1}{\tilde{r}}\left(C_{1} \cos \tilde{r}+\frac{C_{2}}{2} \sin \tilde{r}+1\right) .
$$

Really from the equation

$$
\frac{\partial \tilde{B}}{\partial \tilde{r}}=-\frac{1}{\tilde{r}^{2}}\left(C_{1} \cos \tilde{r}+\frac{C_{2}}{2} \sin \tilde{r}+1\right)+\frac{1}{\tilde{r}}\left(-C_{1} \sin \tilde{r}+\frac{C_{2}}{2} \cos \tilde{r}\right)
$$

we find

$$
\frac{1}{\tilde{r}}\left(C_{1} \cos \tilde{r}+\frac{C_{2}}{2} \sin \tilde{r}+1\right)+\left(C_{1} \sin \tilde{r}-\frac{C_{2}}{2} \cos \tilde{r}\right)=0 .
$$

If $\tilde{r} \rightarrow \infty$ we have for the long-range order (for the case $C=1$ )

$$
\tan \tilde{r}=\frac{C_{2}}{2 C_{1}}
$$

or

$$
\tilde{r}=\arctan \left[\frac{C_{2}}{2 C_{1}}\right]+\pi n, n=0,1,2, \cdots
$$

It is known that short-range order is the order in the mutual arrangement of atoms or molecules in a substance, which (in contrast to the long-range order) is repeated only at distances commensurate with the distances between atoms, that is, the short-range order is the presence of a pattern in the arrangement of neighboring atoms or molecules. As you see Figures 1-7 demonstrate the

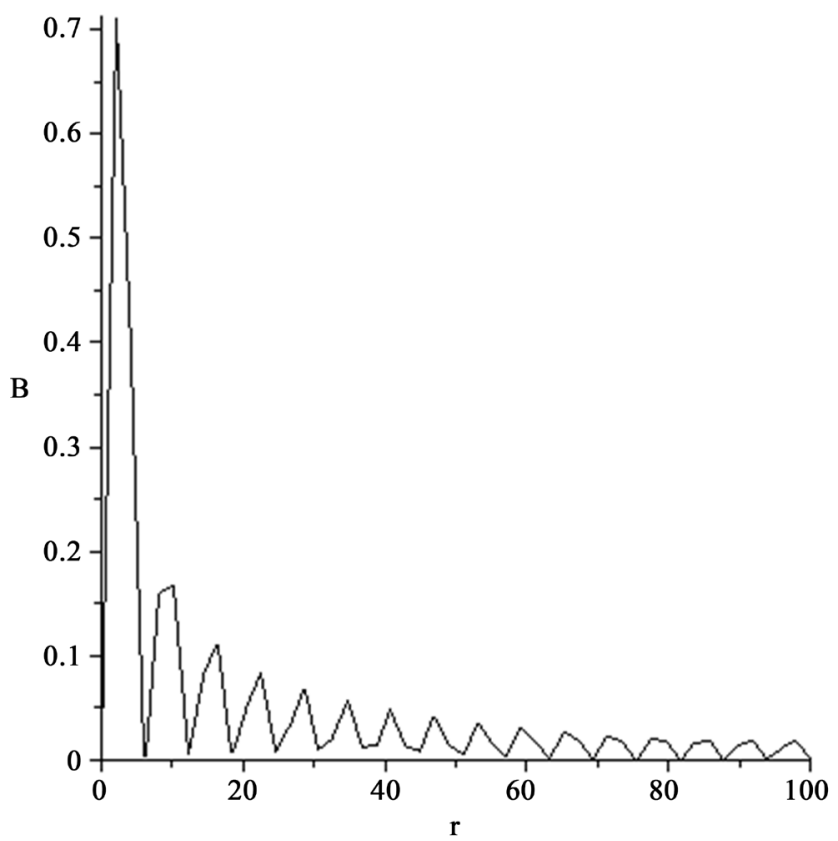

Figure 1. The dependence $\tilde{B}(\tilde{r}), C=1$. 


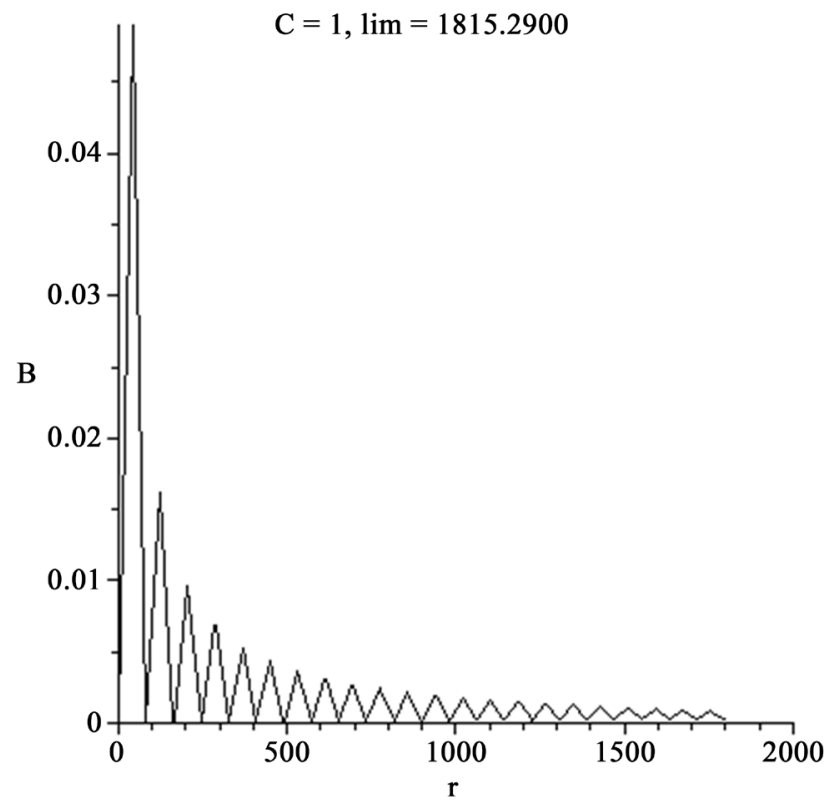

Figure 2. The dependence $\tilde{B}(\tilde{r}), \quad C=1 ; \lim =1815.2900$.

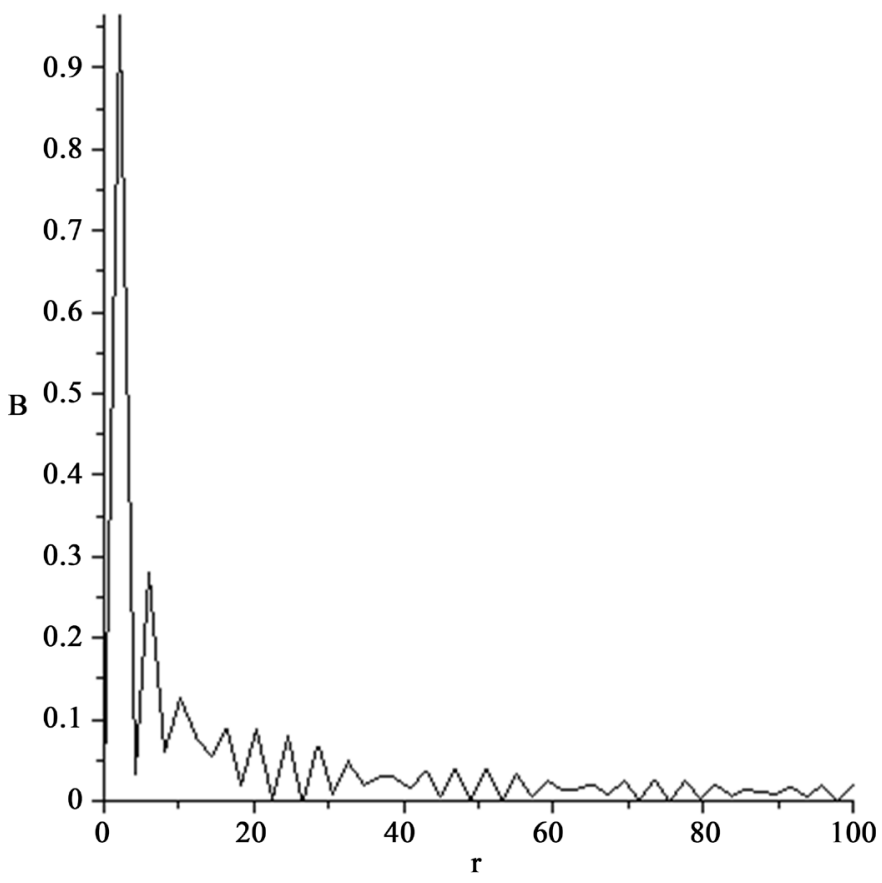

Figure 3. The dependence $\tilde{B}(\tilde{r}), C=2$.

appearance of short- and long-range order.

Obviously we can use the simple Maple program

dsolve[interactive] $\left(\left\{r^{\star} \operatorname{diff}(B(r), r \$ 2)+2^{\star} \operatorname{diff}(B(r), r)+C^{\star} r^{\star} B(r)-C=0, B(0)=0\right.\right.$, $\left.\left.\mathrm{D}(\mathrm{B})(0)=0.5^{\star} \mathrm{C}\right\}\right)$;

We find results reflected on Figure $1(C=1)$ - Figure $7(C=100)$.

Figure 2 reveals the finite domain of the $\tilde{B}(\tilde{r})$ evolution. It should be noticed that the Maple plotter hides the details of the picture at large values $\tilde{r}$. 


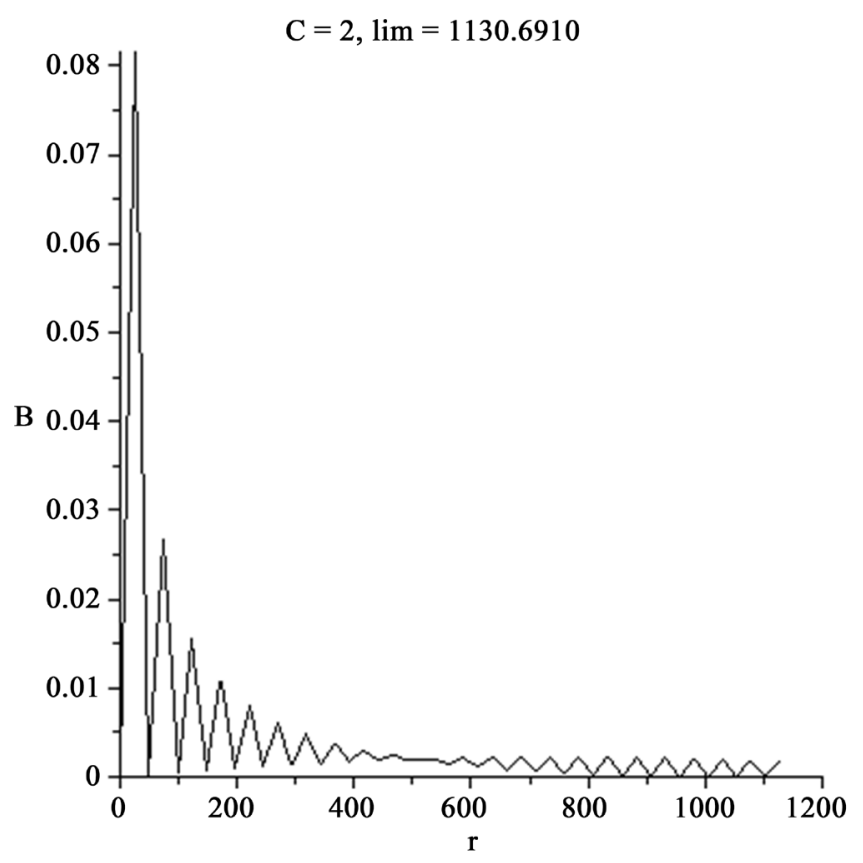

Figure 4. The dependence $\tilde{B}(\tilde{r}), \quad C=2 ; \lim =1130.6910$.

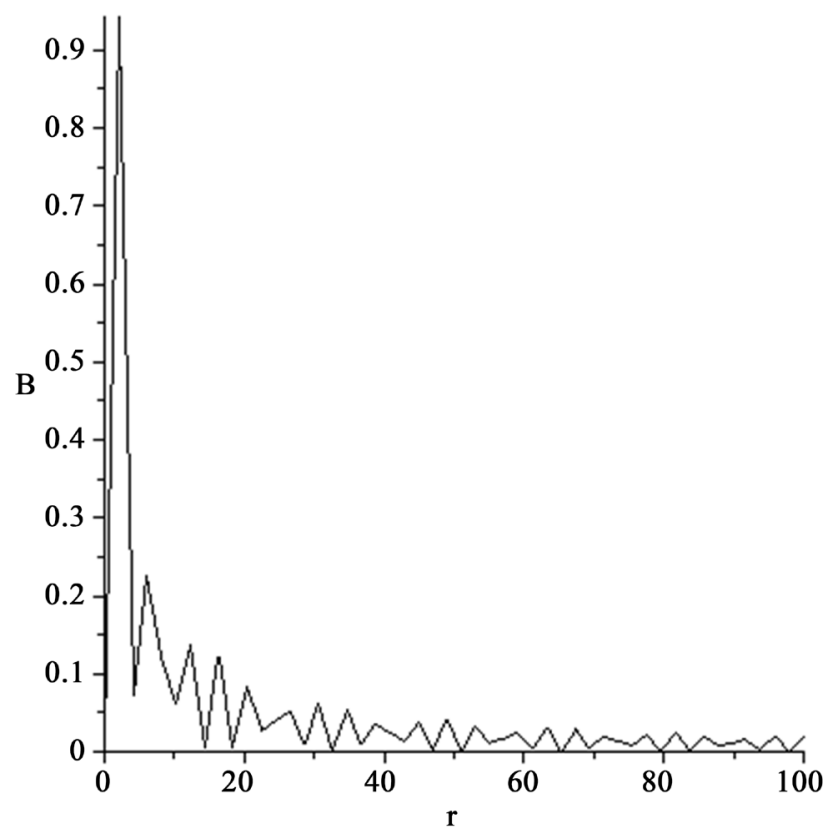

Figure 5. The dependence $\tilde{B}(\tilde{r}), C=3$.

From Figures 1-7 follow:

1) The magnetic field distribution in a superconductor Type II has the character of damping waves.

2) There are modes when vibrational beats appear in a system of damped waves, Figures 3-5.

3) The solutions exist in the limit domain (in contrast to Abrikosov's theory).

4) As we see we reveal the quantization picture of the magnetic field. 


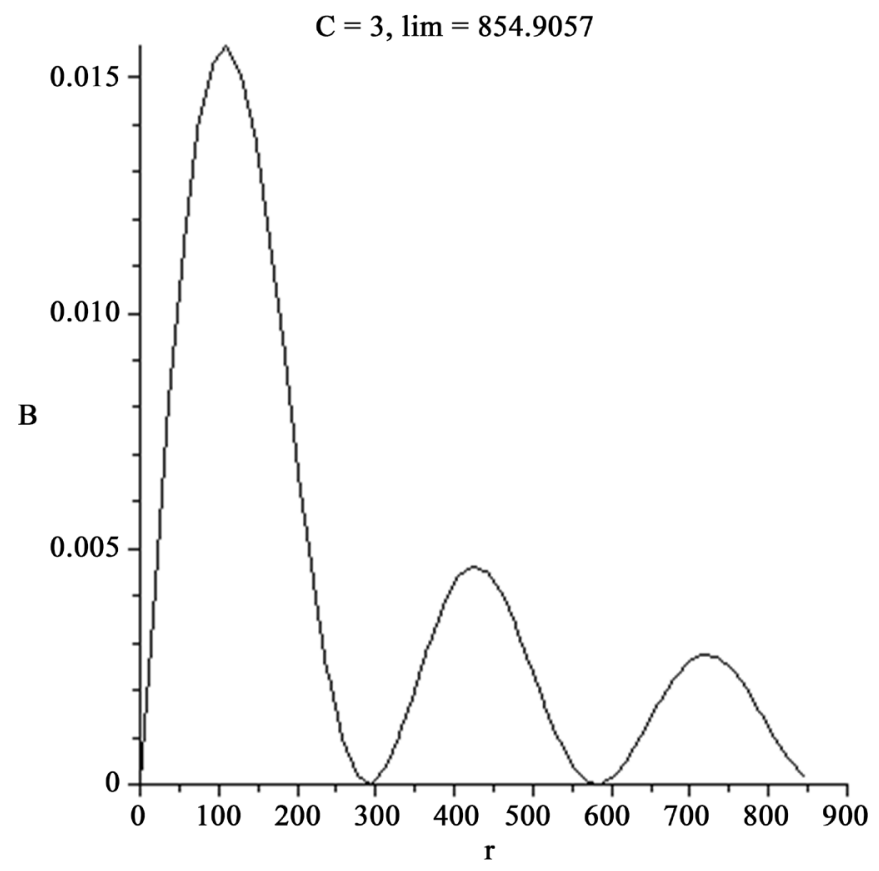

Figure 6. The dependence $\tilde{B}(\tilde{r}), \quad C=3 ; \lim =854.9057$.

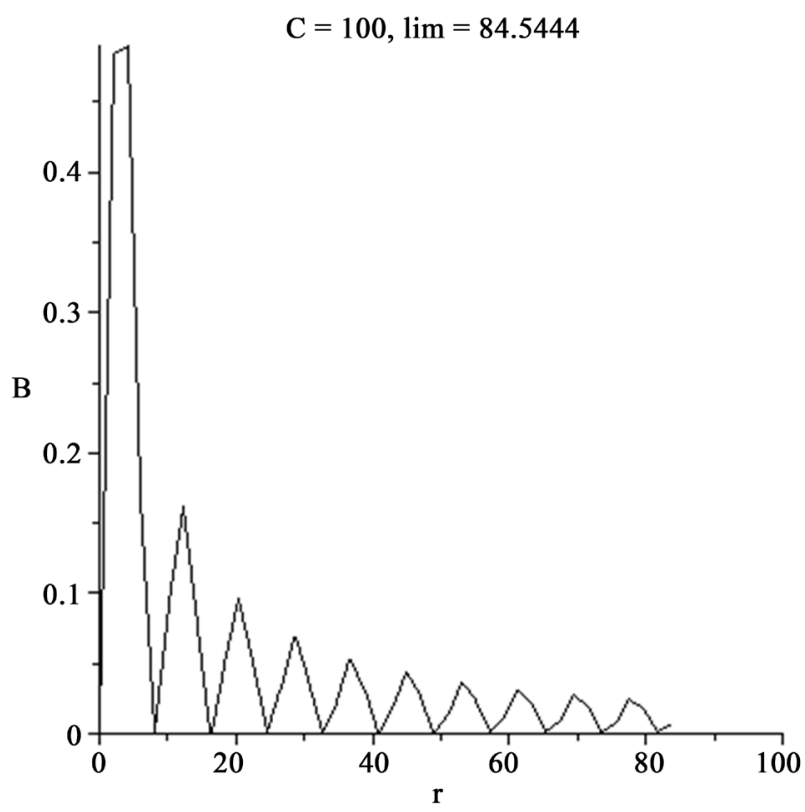

Figure 7. The dependence $\tilde{B}(\tilde{r}), C=100 ; \lim =84.5444$.

\section{Generalized Hydrodynamic Equations}

The generalized hydrodynamic equations (GHE) can be obtained from the nonlocal kinetic equation in the frame of the Enskog procedure, [2] [3] [4]. Generally speaking to GHE should be added the system of generalized Maxwell equations (for example in the form of the generalized Poisson equation for electric potential) and gravitational equations (for example in the form of the generalized Poisson equation for gravitational potential). For example 


$$
\frac{\partial}{\partial \mathbf{r}} \cdot \mathbf{F}^{(1)}=-4 \pi \gamma_{N}\left[\rho-\tau\left(\frac{\partial \rho}{\partial t}+\frac{\partial}{\partial \mathbf{r}} \cdot \rho \mathbf{v}_{0}\right)\right] .
$$

(Continuity equation for species $\alpha$ )

$$
\begin{aligned}
& \frac{\partial}{\partial t}\left\{\rho_{\alpha}-\tau_{\alpha}\left[\frac{\partial \rho_{\alpha}}{\partial t}+\frac{\partial}{\partial \mathbf{r}} \cdot\left(\rho_{\alpha} \mathbf{v}_{0}\right)\right]\right\}+\frac{\partial}{\partial \mathbf{r}} \cdot\left\{\rho_{\alpha} \mathbf{v}_{0}-\tau_{\alpha}\left[\frac{\partial}{\partial t}\left(\rho_{\alpha} \mathbf{v}_{0}\right)\right.\right. \\
& \left.\left.+\frac{\partial}{\partial \mathbf{r}} \cdot\left(\rho_{\alpha} \mathbf{v}_{0} \mathbf{v}_{0}\right)+\overrightarrow{\mathrm{I}} \cdot \frac{\partial p_{\alpha}}{\partial \mathbf{r}}-\rho_{\alpha} \mathbf{F}_{\alpha}^{(1)}-\frac{q_{\alpha}}{m_{\alpha}} \rho_{\alpha} \mathbf{v}_{0} \times \mathbf{B}\right]\right\}=R_{\alpha} .
\end{aligned}
$$

(Continuity equation for mixture)

$$
\begin{aligned}
& \frac{\partial}{\partial t}\left\{\rho-\sum_{\alpha} \tau_{\alpha}\left[\frac{\partial \rho_{\alpha}}{\partial t}+\frac{\partial}{\partial \mathbf{r}} \cdot\left(\rho_{\alpha} \mathbf{v}_{0}\right)\right]\right\}+\frac{\partial}{\partial \mathbf{r}} \cdot\left\{\rho \mathbf{v}_{0}-\sum_{\alpha} \tau_{\alpha}\left[\frac{\partial}{\partial t}\left(\rho_{\alpha} \mathbf{v}_{0}\right)\right.\right. \\
& \left.\left.+\frac{\partial}{\partial \mathbf{r}} \cdot\left(\rho_{\alpha} \mathbf{v}_{0} \mathbf{v}_{0}\right)+\overrightarrow{\mathrm{I}} \cdot \frac{\partial p_{\alpha}}{\partial \mathbf{r}}-\rho_{\alpha} \mathbf{F}_{\alpha}^{(1)}-\frac{q_{\alpha}}{m_{\alpha}} \rho_{\alpha} \mathbf{v}_{0} \times \mathbf{B}\right]\right\}=0 .
\end{aligned}
$$

(Momentum equation for species $\alpha$ )

$$
\begin{aligned}
& \frac{\partial}{\partial t}\left\{\rho_{\alpha} \mathbf{v}_{0}-\tau_{\alpha}\left[\frac{\partial}{\partial t}\left(\rho_{\alpha} \mathbf{v}_{0}\right)+\frac{\partial}{\partial \mathbf{r}} \cdot \rho_{\alpha} \mathbf{v}_{0} \mathbf{v}_{0}+\frac{\partial p_{\alpha}}{\partial \mathbf{r}}-\rho_{\alpha} \mathbf{F}_{\alpha}^{(1)}\right.\right. \\
& \left.\left.-\frac{q_{\alpha}}{m_{\alpha}} \rho_{\alpha} \mathbf{v}_{0} \times \mathbf{B}\right]\right\}-\mathbf{F}_{\alpha}^{(1)}\left[\rho_{\alpha}-\tau_{\alpha}\left(\frac{\partial \rho_{\alpha}}{\partial t}+\frac{\partial}{\partial \mathbf{r}} \cdot\left(\rho_{\alpha} \mathbf{v}_{0}\right)\right]\right] \\
& -\frac{q_{\alpha}}{m_{\alpha}}\left\{\rho_{\alpha} \mathbf{v}_{0}-\tau_{\alpha}\left[\frac{\partial}{\partial t}\left(\rho_{\alpha} \mathbf{v}_{0}\right)+\frac{\partial}{\partial \mathbf{r}} \cdot \rho_{\alpha} \mathbf{v}_{0} \mathbf{v}_{0}+\frac{\partial p_{\alpha}}{\partial \mathbf{r}}-\rho_{\alpha} \mathbf{F}_{\alpha}^{(1)}\right.\right. \\
& \left.\left.-\frac{q_{\alpha}}{m_{\alpha}} \rho_{\alpha} \mathbf{v}_{0} \times \mathbf{B}\right]\right\} \times \mathbf{B}+\frac{\partial}{\partial \mathbf{r}} \cdot\left\{\rho_{\alpha} \mathbf{v}_{0} \mathbf{v}_{0}+p_{\alpha} \overrightarrow{\mathrm{I}}-\tau_{\alpha}\left[\frac { \partial } { \partial t } \left(\rho_{\alpha} \mathbf{v}_{0} \mathbf{v}_{0}\right.\right.\right. \\
& \left.+p_{\alpha} \overrightarrow{\mathrm{I}}\right)+\frac{\partial}{\partial \mathbf{r}} \cdot \rho_{\alpha}\left(\mathbf{v}_{0} \mathbf{v}_{0}\right) \mathbf{v}_{0}+2 \overrightarrow{\mathrm{I}}\left(\frac{\partial}{\partial \mathbf{r}} \cdot\left(p_{\alpha} \mathbf{v}_{0}\right)\right)+\frac{\partial}{\partial \mathbf{r}} \cdot\left(\overleftrightarrow{\mathrm{I}} p_{\alpha} \mathbf{v}_{0}\right) \\
& \left.\left.-\mathbf{F}_{\alpha}^{(1)} \rho_{\alpha} \mathbf{v}_{0}-\rho_{\alpha} \mathbf{v}_{0} \mathbf{F}_{\alpha}^{(1)}-\frac{q_{\alpha}}{m_{\alpha}} \rho_{\alpha}\left[\mathbf{v}_{0} \times \mathbf{B}\right] \mathbf{v}_{0}-\frac{q_{\alpha}}{m_{\alpha}} \rho_{\alpha} \mathbf{v}_{0}\left[\mathbf{v}_{0} \times \mathbf{B}\right]\right]\right\} \\
& =\int m_{\alpha} \mathbf{v}_{\alpha} J_{\alpha}^{s t, e l} \mathrm{~d} \mathbf{v}_{\alpha}+\int m_{\alpha} \mathbf{v}_{\alpha} J_{\alpha}^{\text {st, inel }} \mathrm{d} \mathbf{v}_{\alpha} .
\end{aligned}
$$

(Momentum equation for mixture)

$$
\begin{aligned}
& \frac{\partial}{\partial t}\left\{\rho \mathbf{v}_{0}-\sum_{\alpha} \tau_{\alpha}\left[\frac{\partial}{\partial t}\left(\rho_{\alpha} \mathbf{v}_{0}\right)+\frac{\partial}{\partial \mathbf{r}} \cdot \rho_{\alpha} \mathbf{v}_{0} \mathbf{v}_{0}+\frac{\partial p_{\alpha}}{\partial \mathbf{r}}-\rho_{\alpha} \mathbf{F}_{\alpha}^{(1)}\right.\right. \\
& \left.\left.-\frac{q_{\alpha}}{m_{\alpha}} \rho_{\alpha} \mathbf{v}_{0} \times \mathbf{B}\right]\right\}-\sum_{\alpha} \mathbf{F}_{\alpha}^{(1)}\left[\rho_{\alpha}-\tau_{\alpha}\left(\frac{\partial \rho_{\alpha}}{\partial t}+\frac{\partial}{\partial \mathbf{r}} \cdot\left(\rho_{\alpha} \mathbf{v}_{0}\right)\right)\right] \\
& -\sum_{\alpha} \frac{q_{\alpha}}{m_{\alpha}}\left\{\rho_{\alpha} \mathbf{v}_{0}-\tau_{\alpha}\left[\frac{\partial}{\partial t}\left(\rho_{\alpha} \mathbf{v}_{0}\right)+\frac{\partial}{\partial \mathbf{r}} \cdot \rho_{\alpha} \mathbf{v}_{0} \mathbf{v}_{0}+\frac{\partial p_{\alpha}}{\partial \mathbf{r}}-\rho_{\alpha} \mathbf{F}_{\alpha}^{(1)}\right.\right. \\
& \left.\left.-\frac{q_{\alpha}}{m_{\alpha}} \rho_{\alpha} \mathbf{v}_{0} \times \mathbf{B}\right]\right\} \times \mathbf{B}+\frac{\partial}{\partial \mathbf{r}} \cdot\left\{\rho \mathbf{v}_{0} \mathbf{v}_{0}+p \overrightarrow{\mathrm{I}}-\sum_{\alpha} \tau_{\alpha}\left[\frac { \partial } { \partial t } \left(\rho_{\alpha} \mathbf{v}_{0} \mathbf{v}_{0}\right.\right.\right. \\
& \left.+p_{\alpha} \overrightarrow{\mathrm{I}}\right)+\frac{\partial}{\partial \mathbf{r}} \cdot \rho_{\alpha}\left(\mathbf{v}_{0} \mathbf{v}_{0}\right) \mathbf{v}_{0}+2 \ddot{\mathrm{I}}\left(\frac{\partial}{\partial \mathbf{r}} \cdot\left(p_{\alpha} \mathbf{v}_{0}\right)\right)+\frac{\partial}{\partial \mathbf{r}} \cdot\left(\left(\overrightarrow{\mathrm{I}} p_{\alpha} \mathbf{v}_{0}\right)\right. \\
& \left.\left.-\mathbf{F}_{\alpha}^{(1)} \rho_{\alpha} \mathbf{v}_{0}-\rho_{\alpha} \mathbf{v}_{0} \mathbf{F}_{\alpha}^{(1)}-\frac{q_{\alpha}}{m_{\alpha}} \rho_{\alpha}\left[\mathbf{v}_{0} \times \mathbf{B}\right] \mathbf{v}_{0}-\frac{q_{\alpha}}{m_{\alpha}} \rho_{\alpha} \mathbf{v}_{0}\left[\mathbf{v}_{0} \times \mathbf{B}\right]\right]\right\}=0 .
\end{aligned}
$$


(Energy equation for $\alpha$ species)

$$
\begin{aligned}
& \frac{\partial}{\partial t}\left\{\frac{\rho_{\alpha} v_{0}^{2}}{2}+\frac{3}{2} p_{\alpha}+\varepsilon_{\alpha} n_{\alpha}-\tau_{\alpha}\left[\frac{\partial}{\partial t}\left(\frac{\rho_{\alpha} v_{0}^{2}}{2}+\frac{3}{2} p_{\alpha}+\varepsilon_{\alpha} n_{\alpha}\right)\right.\right. \\
& \left.\left.+\frac{\partial}{\partial \mathbf{r}} \cdot\left(\frac{1}{2} \rho_{\alpha} v_{0}^{2} \mathbf{v}_{0}+\frac{5}{2} p_{\alpha} \mathbf{v}_{0}+\varepsilon_{\alpha} n_{\alpha} \mathbf{v}_{0}\right)-\mathbf{F}_{\alpha}^{(1)} \cdot \rho_{\alpha} \mathbf{v}_{0}\right]\right\} \\
& +\frac{\partial}{\partial \mathbf{r}} \cdot\left\{\frac{1}{2} \rho_{\alpha} v_{0}^{2} \mathbf{v}_{0}+\frac{5}{2} p_{\alpha} \mathbf{v}_{0}+\varepsilon_{\alpha} n_{\alpha} \mathbf{v}_{0}-\tau_{\alpha}\left[\frac { \partial } { \partial t } \left(\frac{1}{2} \rho_{\alpha} v_{0}^{2} \mathbf{v}_{0}\right.\right.\right. \\
& \left.+\frac{5}{2} p_{\alpha} \mathbf{v}_{0}+\varepsilon_{\alpha} n_{\alpha} \mathbf{v}_{0}\right)+\frac{\partial}{\partial \mathbf{r}} \cdot\left(\frac{1}{2} \rho_{\alpha} v_{0}^{2} \mathbf{v}_{0} \mathbf{v}_{0}+\frac{7}{2} p_{\alpha} \mathbf{v}_{0} \mathbf{v}_{0}+\frac{1}{2} p_{\alpha} v_{0}^{2} \overrightarrow{\mathrm{I}}\right. \\
& \left.+\frac{5}{2} \frac{p_{\alpha}^{2}}{\rho_{\alpha}} \overrightarrow{\mathrm{I}}+\varepsilon_{\alpha} n_{\alpha} \mathbf{v}_{0} \mathbf{v}_{0}+\varepsilon_{\alpha} \frac{p_{\alpha}}{m_{\alpha}} \overrightarrow{\mathrm{I}}\right)-\rho_{\alpha} \mathbf{F}_{\alpha}^{(1)} \cdot \mathbf{v}_{0} \mathbf{v}_{0}-p_{\alpha} \mathbf{F}_{\alpha}^{(1)} \cdot \overrightarrow{\mathrm{I}} \\
& -\frac{1}{2} \rho_{\alpha} v_{0}^{2} \mathbf{F}_{\alpha}^{(1)}-\frac{3}{2} \mathbf{F}_{\alpha}^{(1)} p_{\alpha}-\frac{\rho_{\alpha} v_{0}^{2}}{2} \frac{q_{\alpha}}{m_{\alpha}}\left[\mathbf{v}_{0} \times \mathbf{B}\right]-\frac{5}{2} p_{\alpha} \frac{q_{\alpha}}{m_{\alpha}}\left[\mathbf{v}_{0} \times \mathbf{B}\right] \\
& \left.\left.-\varepsilon_{\alpha} n_{\alpha} \frac{q_{\alpha}}{m_{\alpha}}\left[\mathbf{v}_{0} \times \mathbf{B}\right]-\varepsilon_{\alpha} n_{\alpha} \mathbf{F}_{\alpha}^{(1)}\right]\right\}-\left\{\rho_{\alpha} \mathbf{F}_{\alpha}^{(1)} \cdot \mathbf{v}_{0}-\tau_{\alpha}\left[\mathbf { F } _ { \alpha } ^ { ( 1 ) } \cdot \left(\frac{\partial}{\partial t}\left(\rho_{\alpha} \mathbf{v}_{0}\right)\right.\right.\right. \\
& \left.\left.\left.+\frac{\partial}{\partial \mathbf{r}} \cdot \rho_{\alpha} \mathbf{v}_{0} \mathbf{v}_{0}+\frac{\partial}{\partial \mathbf{r}} \cdot p_{\alpha} \overrightarrow{\mathrm{I}}-\rho_{\alpha} \mathbf{F}_{\alpha}^{(1)}-q_{\alpha} n_{\alpha}\left[\mathbf{v}_{0} \times \mathbf{B}\right]\right)\right]\right\} \\
& =\int\left(\frac{m_{\alpha} v_{\alpha}^{2}}{2}+\varepsilon_{\alpha}\right) J_{\alpha}^{s t, e l} \mathrm{~d} \mathbf{v}_{\alpha}+\int\left(\frac{m_{\alpha} v_{\alpha}^{2}}{2}+\varepsilon_{\alpha}\right) J_{\alpha}^{\text {st,inel }} \mathrm{d} \mathbf{v}_{\alpha} .
\end{aligned}
$$

(Energy equation for mixture)

$$
\begin{aligned}
& \frac{\partial}{\partial t}\left\{\frac{\rho v_{0}^{2}}{2}+\frac{3}{2} p+\sum_{\alpha} \varepsilon_{\alpha} n_{\alpha}-\sum_{\alpha} \tau_{\alpha}\left[\frac{\partial}{\partial t}\left(\frac{\rho_{\alpha} v_{0}^{2}}{2}+\frac{3}{2} p_{\alpha}+\varepsilon_{\alpha} n_{\alpha}\right)\right.\right. \\
& \left.\left.+\frac{\partial}{\partial \mathbf{r}} \cdot\left(\frac{1}{2} \rho_{\alpha} v_{0}^{2} \mathbf{v}_{0}+\frac{5}{2} p_{\alpha} \mathbf{v}_{0}+\varepsilon_{\alpha} n_{\alpha} \mathbf{v}_{0}\right)-\mathbf{F}_{\alpha}^{(1)} \cdot \rho_{\alpha} \mathbf{v}_{0}\right]\right\} \\
& +\frac{\partial}{\partial \mathbf{r}} \cdot\left\{\frac{1}{2} \rho v_{0}^{2} \mathbf{v}_{0}+\frac{5}{2} p \mathbf{v}_{0}+\mathbf{v}_{0} \sum \varepsilon_{\alpha} n_{\alpha}-\sum_{\alpha} \tau_{\alpha}\left[\frac { \partial } { \partial t } \left(\frac{1}{2} \rho_{\alpha} v_{0}^{2} \mathbf{v}_{0}\right.\right.\right. \\
& \left.+\frac{5}{2} p_{\alpha} \mathbf{v}_{0}+\varepsilon_{\alpha} n_{\alpha} \mathbf{v}_{0}\right)+\frac{\partial}{\partial \mathbf{r}} \cdot\left(\frac{1}{2} \rho_{\alpha} v_{0}^{2} \mathbf{v}_{0} \mathbf{v}_{0}+\frac{7}{2} p_{\alpha} \mathbf{v}_{0} \mathbf{v}_{0}+\frac{1}{2} p_{\alpha} v_{0}^{2} \overrightarrow{\mathrm{I}}\right. \\
& \left.+\frac{5}{2} \frac{p_{\alpha}^{2}}{\rho_{\alpha}} \overrightarrow{\mathrm{I}}+\varepsilon_{\alpha} n_{\alpha} \mathbf{v}_{0} \mathbf{v}_{0}+\varepsilon_{\alpha} \frac{p_{\alpha}}{m_{\alpha}} \overrightarrow{\mathrm{I}}\right)-\rho_{\alpha} \mathbf{F}_{\alpha}^{(1)} \cdot \mathbf{v}_{0} \mathbf{v}_{0}-p_{\alpha} \mathbf{F}_{\alpha}^{(1)} \cdot \overrightarrow{\mathrm{I}} \\
& -\frac{1}{2} \rho_{\alpha} v_{0}^{2} \mathbf{F}_{\alpha}^{(1)}-\frac{3}{2} \mathbf{F}_{\alpha}^{(1)} p_{\alpha}-\frac{\rho_{\alpha} v_{0}^{2}}{2} \frac{q_{\alpha}}{m_{\alpha}}\left[\mathbf{v}_{0} \times \mathbf{B}\right]-\frac{5}{2} p_{\alpha} \frac{q_{\alpha}}{m_{\alpha}}\left[\mathbf{v}_{0} \times \mathbf{B}\right] \\
& \left.\left.-\varepsilon_{\alpha} n_{\alpha} \frac{q_{\alpha}}{m_{\alpha}}\left[\mathbf{v}_{0} \times \mathbf{B}\right]-\varepsilon_{\alpha} n_{\alpha} \mathbf{F}_{\alpha}^{(1)}\right]\right\}-\left\{\mathbf{v}_{0} \cdot \sum_{\alpha} \rho_{\alpha} \mathbf{F}_{\alpha}^{(1)}-\sum_{\alpha} \tau_{\alpha}\left[\mathbf { F } _ { \alpha } ^ { ( 1 ) } \cdot \left(\frac{\partial}{\partial t}\left(\rho_{\alpha} \mathbf{v}_{0}\right)\right.\right.\right. \\
& \left.\left.\left.+\frac{\partial}{\partial \mathbf{r}} \cdot \rho_{\alpha} \mathbf{v}_{0} \mathbf{v}_{0}+\frac{\partial}{\partial \mathbf{r}} \cdot p_{\alpha} \overrightarrow{\mathrm{I}}-\rho_{\alpha} \mathbf{F}_{\alpha}^{(1)}-q_{\alpha} n_{\alpha}\left[\mathbf{v}_{0} \times \mathbf{B}\right]\right)\right]\right\}=0 . \\
&
\end{aligned}
$$

Here $\mathbf{F}_{\alpha}^{(1)}$ are the forces of the non-magnetic origin, $\mathbf{B}-$ magnetic induction, $\overrightarrow{\mathrm{I}}$-unit tensor, $q_{\alpha}$-charge of the $\alpha$-component particle, $p_{\alpha}$-static pressure for $\alpha$-component, $\varepsilon_{\alpha}$-internal energy for the particles of $\alpha$-com- 
ponent, $\mathbf{v}_{0}$-hydrodynamic velocity for mixture, $\tau_{\alpha}$-non-local parameter.

GHE are extremely important for astrophysics special cases when density $\rho \rightarrow 0$ (the initial stage of evolution of the Universe, the Big Bang; transport processes in physical vacuum) and when density $\rho \rightarrow \infty$ (evolution of the black hole). Both limiting cases have no physical or mathematical meaning in "classical" hydrodynamics. Thus, we have a unified statistical theory of dissipative structures, which has a hydrodynamic shape defined by the genesis of GHE. Then we obliged to deliver come comments concerning application of special (SRT) and general (GRT) relativistic theory in theoretical astrophysics.

\section{Nonlocal Physics and Special and General Theory of Relativity}

Let us investigate the possible connection between nonlocal physics and special theory of relativity (SRT). The underlying SRT experiment belongs to Albert A. Michelson and Edward W. Morley. The Michelson-Morley experiment was performed and published in November, 1887 (which has tremendous variants in the following years) [23]. They compared the speed of light in perpendicular directions, in an attempt to detect the relative motion of matter through some stationary medium called aether.

The result was negative, in that the expected difference between the speed of light in the direction of movement through the presumed aether, and the speed at right angles, was found not to exist. Michelson-Morley type experiments have been repeated many times with steadily increasing sensitivity. The result is considered as the evidence against the aether theory, and initiated a line of research that eventually led to special relativity, which rules out a stationary aether. Figure 8 shows the Michelson, Morley device. Calculate the time for which the light will pass the distance to the mirror in the horizontal direction. In one second light travels $c$ meters, and the aether wind blows it in $v$ meters back. Therefore, the actual speed will be equal $(c-v)$. It means that light will reach the mirror over $t=\frac{L \Rightarrow}{c-v}$.

Obviously the way back will take time $t=\frac{L_{\Rightarrow}}{c+v}$. Total time spent:

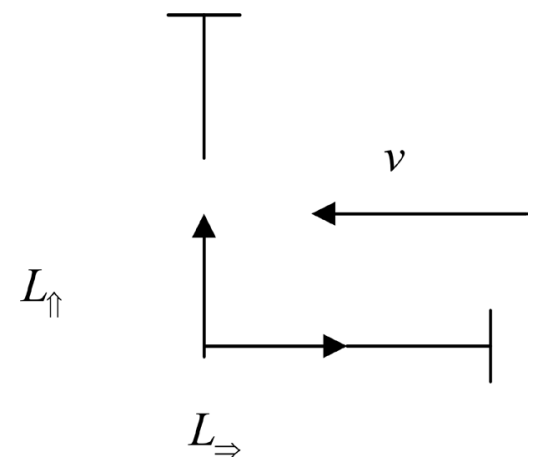

Figure 8. The principal scheme of Michelson - Morley experiment. 


$$
t_{1}=\frac{L_{\Rightarrow}}{c+v}+\frac{L_{\Rightarrow}}{c-v}=\frac{2 L_{\Rightarrow} c}{c^{2}-v^{2}} .
$$

Calculate the elapsed time for moving in the vertical direction. In one second the aether wind will shift the light on the $v$ meters to the left.

In other words the stationary observer should see the real velocity of the vertical movement as $u^{2}+v^{2}=c^{2}$, or $u=c \cos \phi=\sqrt{c^{2}-v^{2}}$. Then the real rate of convergence of the light with mirror (reflected on Figure 8 as the upper mirror) is $u=\sqrt{c^{2}-v^{2}}$. Moving in the opposite direction leads to the symmetrical situation. Thus,

$$
t_{2}=\frac{2 L_{\Uparrow}}{\sqrt{c^{2}-v^{2}}} .
$$

The time difference is

$$
\begin{aligned}
t_{1}-t_{2} & =\frac{2 L_{\Rightarrow} C}{c^{2}-v^{2}}-\frac{2 L_{\Uparrow}}{\sqrt{c^{2}-v^{2}}}=\frac{2 c}{c^{2}-v^{2}}\left[L_{\Rightarrow}-L_{\Uparrow} \frac{\sqrt{c^{2}-v^{2}}}{c}\right] \\
& =\frac{2 c}{c^{2}-v^{2}}\left[L_{\Rightarrow}-L_{\Uparrow} \sqrt{1-\frac{v^{2}}{c^{2}}}\right] .
\end{aligned}
$$

\section{Some conclusions:}

1) The mentioned above configuration known as Michelson-Morley experiment (1887) would have detected the earth's motion through the supposed luminiferous aether that most physicists at the time believed was the medium in which light waves propagated. From the first glance the null result of that experiment leads to the condition (see (6.3))

$$
t_{1}-t_{2}=0 \text {. }
$$

2) But relation (6.4) obtained for the closed thermodynamic system without taking into account the direct influence of physical vacuum PV (or in old terminology) luminiferous aether.

$3)$ In principal we consider the open thermodynamic system interacting with physical vacuum (PV). But the relation (6.1)-(6.3) obtained for classic dynamic system without taking into account the direct influence of PV on the mentioned system.

4) Nevertheless we intend to construct simplified theory excluding the direct $\mathrm{PV}$ influence. This fact leads to kinematic relation

$$
L_{\Rightarrow}=L_{\Uparrow} \sqrt{1-\frac{v^{2}}{c^{2}}} .
$$

Let us consider this situation in detail from the position of the nonlocal physics. The Michelson installation can be considered as a device delivering particles moving with the velocities $v$ in the open probe contour filled by PV. The corresponding nonlocal parameter is (see also (2.7))

$$
\tau_{D}=\frac{H_{D}}{v^{2}} .
$$


For photons we have ( $v_{f}$ is frequency)

$$
\tau_{p h} h v_{f}=h,
$$

or

$$
\tau_{p h}=\frac{1}{v_{f}}
$$

Now we can rewrite the relation (6.3) in the form

$$
t_{1}-t_{2}=\frac{2 c}{c^{2}-v^{2}}\left[L_{\Rightarrow}-L_{\Uparrow} \sqrt{1-\frac{v^{2}}{c^{2}}}\right]=\frac{2 c \tau_{D}}{\tau_{D} c^{2}-H_{D}}\left[L_{\Rightarrow}-L_{\Uparrow} \sqrt{1-\frac{H_{D}}{\tau_{D} c^{2}}}\right] .
$$

Michelson's experiment assumes that the length of the instrument's shoulders does not change when rotated to a right angle.

In this case from the position of nonlocal physics

$$
t_{1}-t_{2}=0
$$

only if

$$
H_{D} \rightarrow 0
$$

or if the PV viscosity tends to zero. As we see this affirmation contradicts the Heisenberg's uncertainty principle.

Moreover, it could be said that the uncertainty principle reflects the existence of Physical Vacuum.

From the physical point of view it means that for the realizations (6.10) we should exclude the influence of PV in the explicit form. Then (if $H_{D} \rightarrow 0$ )

$$
t_{1}-t_{2} \rightarrow \frac{2}{c}\left[L_{\Rightarrow}-L_{\Uparrow}\right]=0 \text {. }
$$

H.A. Lorentz was aware in the aether existence. In his lectures on theoretical physics delivered at the University of Leiden he wrote [24], (Chapter 1, Paragraph 8: "Reduction in the direction of movement") he wrote:

“The negative result of Michelson's experiment can be explained by assuming that the length of the instrument's shoulders changes when rotated to a right angle....This size dependence on the orientation relative to the Earth's motion is not as surprising as it might seem at first. Indeed, the size is determined by molecular forces, and since the latter are transmitted over the aether, on the contrary it would be strange if the state of motion of the latter does not affect the size of bodies. The nature of molecular forces unknown to us; however, if we assume that they are transmitted through the aether in the same way as the electrical forces, then we can build a theory of this reduction, which gives the value of the latter, exactly corresponding to the value necessary to explain the zero effect of the Michelson's experiment. The size of this reduction will be $6.5 \mathrm{~cm}$ for the diameter of the Earth and 1/200 microns for the rod length of $1 \mathrm{~m}$."

No difficulties to reproduce the Lorentz' estimation; for example for the rod reduction $L_{r r}=\sqrt{1-\frac{9 \times 10^{2}}{9 \times 10^{10}}}=L\left(1-\frac{1}{2} \times 10^{-8}\right)$, m we have $\left(v_{\text {earth }}=30 \mathrm{~km} / \mathrm{s}\right.$, 
$c=3 \times 10^{5} \mathrm{~km} / \mathrm{s}$.

The origin of contradiction consists in situation when the open thermodynamic system is considered as a closed one.

In other words the mirrors should not be considered as fixed objects.

Then special theory of relativity is kinematic theory which allows avoiding PV effects in the explicit form from consideration.

This affirmation is valid for the situation when the ratio of the object velocity to the light velocity in an inertial coordinate system is not small.

Criticism of general relativity (GR) is well known topic in theoretical physics (see for example [25]). No reason to discuss here the shortcomings of GRT. Nevertheless we should notice:

1) GRT does not contain the conservation laws, in other words GRT can't serve as a basement for investigation of transport processes in physical systems.

2) GRT is a phenomenological generalization of the Poisson equation with the aim to organize the transfer to the Newtonian gravitation. Not surprisingly, this equation has wave solutions. It was meant to be, not the other way around.

3) Poisson equation has no interpretation even in Madelung-Schrödinger quantum mechanics (QM). Then we have no chance to obtain the unified GRT-QM theory.

4) GRT postulates that the rate of transmission of gravitational interactions is equal to the speed of light. Laplace and Poincaré [26] [27] believed that the rate of transmission of gravitational interactions is several orders of magnitude higher than the speed of light; extremely important problem since the time of Laplace and Poincaré.

5) Formally speaking the Newtonian gravity propagates with the infinite speed. This conclusion is connected only with the description in the frame of local physics. Usual affirmation - general relativity (GR) reduces to Newtonian gravity in the weak-field, low-velocity limit. In literature you can find criticism of this affirmation because the conservation of angular momentum is implicit in the assumptions on which GR rests. Finite propagation speeds and conservation of angular momentum are incompatible in GR. Therefore, phenomenological GR was forced to claim that gravity is not a force that propagates in any classical sense.

6) In many cases, the result of the calculations is simply adjusted to the desired one using a small number of experimental data (including the situation with the $\Lambda$-term and Soldner problem discussed in [6]).

7) GTR has no reasonable asymptotic behavior at a density tending to infinity (a black hole) and at a density tending to zero (a physical vacuum). The appearance of singularity can not be considered as a reasonable solution.

Then black hole formation is not a robust prediction of the general theory of relativity. The idea of a black hole was known for a hundred years before Einstein.

The following effects of the principal significance exist as a direct consequence 
of nonlocal physics (see monographs [6] [28]):

1) The birth of the Universe is convoying of appearance of the repulsion forces. In the existing terminology we discover the "negative pressure" and "dark energy" in all cases. This fundamental result does not depend on the mechanism of external perturbations. In other words, the anti-gravity in the physical vacuum exists, if there is dissipation of energy or in the absence of dissipation at all.

2) Physical Vacuum (PV) is not a speculative object; it is a reality as "matter" and "fields". In other words, the physical vacuum is "the third" physical reality along with matter and fields.

3) The fundamental statistical theory on the dissipative non-equilibrium theory should be reconsidered.

4) The theoretical basement of the advanced technologies of the $21^{\text {st }}$ century is nonlocal physics.

\section{Hubble Effect and Nonlocal Physics}

A special place in astrophysics is the effect of Hubble-expanding groups of galaxies, accompanied by a proportional increase in the rate of expansion groups based on the distance from the main center of gravity. The proportionality factor is the Hubble constant $\left(\mathrm{Hu}\right.$, for Universe $\mathrm{Hu}=2.3 \times 10^{-18} \mathrm{~s}^{-1}$ ), which as it turns out, is not a constant value, $v=\mathrm{Hu} r$. We introduce the Hubble parameter notation using the first two letters of the surname (as in similarity theory) in order to avoid confusion with the Boltzmann $H$-function.

The main origin of the Hubble effect (including the matter expansion with acceleration) is self-catching of expanding matter by the self-consistent gravitational field in conditions of the weak influence of the central massive bodies, [4] [6].

It would seem that we are dealing with the well studied problem in classical gas dynamics of point explosion. Not at all, the classical dynamics of a point explosion has no a Hubble mode.

Let us consider now the results of so called inflation theory and Hubble observations from the position of nonlocal physics. It was shown by B.V. Alexeev that the following inequality takes place (so called the generalized BoltzmannAlexeev theorem) [3] [4] [7] [8]. One obtains

$$
\frac{\mathrm{d}}{\mathrm{d} t}\left(H-\tau \frac{\mathrm{d} H}{\mathrm{~d} t}\right) \leq 0 .
$$

We introduce the $H^{a}$ - function in accordance with the definition

$$
H^{a}=H-\tau \frac{\mathrm{d} H}{\mathrm{~d} t} .
$$

Then the inequality is valid that makes up the conclusion of the generalized H-theorem,

$$
\frac{\mathrm{d} H^{a}}{\mathrm{~d} t} \leq 0 .
$$


If we suppose that $\tau$ is constant not depending on time, inequality (7.3) can be considered as combination of two principles-Boltzmann's principle

$$
\frac{\mathrm{d} H}{\mathrm{~d} t} \leq 0
$$

and Prigogine's principle [3] [4] [29] [30]

$$
\frac{\mathrm{d}^{2} H}{\mathrm{~d} t^{2}} \geq 0 \text {. }
$$

The reference level for the energy function can be chosen arbitrarily, in this case we use

$$
H-\tau \frac{\mathrm{d} H}{\mathrm{~d} t}=0 .
$$

or

$$
\frac{\mathrm{d} \ln H}{\mathrm{~d} t}=\frac{1}{\tau}
$$

or if nonlocal parameter $\tau=$ const we have

$$
H=H_{0} \mathrm{e}^{\frac{t}{\tau}}
$$

But the Boltzmann $H$ - function is proportional to the velocity $v_{0 r}$, then

$$
v_{0 r}^{2}=\hat{H}_{0} \mathrm{e}^{\frac{t}{\tau}}
$$

or

$$
v_{0 r}=\sqrt{\hat{H}_{0}} \mathrm{e}^{\frac{t}{2 \tau}}
$$

and

$$
2 v_{0 r} \frac{\partial v_{0 r}}{\partial t}=\hat{H}_{0} \mathrm{e}^{\frac{t}{\tau}} \frac{1}{\tau},
$$

or

$$
2 v_{0 r} \frac{\partial v_{0 r}}{\partial t}=v_{0 r}^{2} \frac{1}{\tau},
$$

or

$$
\mathrm{d} v_{0 r}=\frac{1}{2 \tau} v_{0 r} \mathrm{~d} t
$$

or

$$
\mathrm{d} v_{0 r}=\frac{1}{2 \tau} \mathrm{d} r
$$

or for the $\tau=$ const

$$
v_{0 r}=\mathrm{Hu} r \text {, }
$$

where the Hubble coefficient $\mathrm{Hu}$ is introduced

$$
\mathrm{Hu}=\frac{1}{2 \tau} .
$$


Let us comment now so called the inflationary theory of the Universe expansion in the early stages of the evolution of the Universe. It is assumed in the mentioned theory that in the period from $10^{-42} \mathrm{sec}$ to $10^{-36} \mathrm{sec}$ the Universe was in the inflationary stage of its development. Its main feature is the maximum strong negative pressure of the substance, leading to an exponential increase in the kinetic energy of the Universe and its volume by many orders of magnitude [31]. The inflationary model involves replacing the power law of expansion $\sim \sqrt{t}$ with an exponential law $\sim \exp (\mathrm{Hut})$ where $\mathrm{Hu}$ as before is the Hubble constant of the inflationary stage, in a general way, depending on time. The value of the Hubble constant at the inflation stage is taken as $10^{-42} \mathrm{sec}^{-1}-10^{-36} \mathrm{sec}^{-1}$, that is, it gigantic exceeds its current value. Such a law of expansion can be provided by the states of physical fields ("inflation field") corresponding to the equation of state, that is, negative pressure; this stage is called inflationary (from lat. inflatio-inflation).

The model of cosmic inflation has opponents, including Roger Penrose [32]. The arguments of the opponents are that the solutions offered by the inflationary model are only "sweeping the litter under the carpet". The Rodger Penrose consideration contains the inflation theory criticism from the position of local physics.

We should remark:

1) The basic relation of the inflation theory concerning the exponential growth of the early Universe and the Hubble relation are the simplest consequence of the nonlocal physics.

2) The inflation theory has no chance to solve so called "initial value problems" concerning the Planck time and the problems of the relic gravitational.

3) The negative pressure does not exist.

4) The general relativistic theory is not applicable to the early Universe when the density $\rho \rightarrow 0$ and Big Bang corresponds to the explosion of the Physical Vacuum (PV).

5) The early evolution of the PV can not be considered in the frame of local physics in principal.

6) The proportionality coefficient in the Hubble dependence is commonly referred to as the Hubble $(\mathrm{Hu})$ constant. The value of the Hubble constant continues to be refined, and now the most accurate value is close to $70 \mathrm{~km} / \mathrm{s}$ per $1 \mathrm{me}-$ gaparsec (1 MPC is about 3 million light years). For example, if a galaxy is located at a distance of 100 MPC from us, we can expect that it is moving away from our Galaxy at a speed of about $70 \times 100=7000 \mathrm{~km} / \mathrm{s}$. The Hu value is determined from observations of galaxies whose distances are measured without red shift (primarily from the brightest stars or Cepheids). The independent estimates of $\mathrm{Hu}$ give this parameter a value of $66-78 \mathrm{~km} / \mathrm{s}$ per mega parsec. This means that galaxies located at a distance of 100 mega parsecs are moving away from us at a speed of $6600-7800 \mathrm{~km} / \mathrm{s}$. At present (2019), the values obtained by calculating the distances to galaxies from the luminosity of the Cepheids ob- 
served in them on the Hubble space telescope give an estimate of $74.03 \pm 1.42$ $(\mathrm{km} / \mathrm{s}) /$ mega parsec, and the values obtained by measuring the parameters of relic radiation at the Planck space Observatory showed a value of $67.4 \pm 0.5$ $(\mathrm{km} / \mathrm{s}) /$ mega parsec as of 2018 .

7) The problem of estimating $\mathrm{Hu}$ is complicated by the fact that, in addition to the cosmological speeds due to the expansion of the Universe, galaxies still have their own (circular) speeds, which can be several hundred $\mathrm{km} / \mathrm{s}$ (for members of massive clusters of galaxies-more than $1000 \mathrm{~km} / \mathrm{s}$ ). This leads to the fact that Hubble's law is poorly fulfilled or not fulfilled at all for objects located at a distance closer than 10 - 15 million LY, that is, just for those galaxies whose distances are most reliably determined without redshift. As we see the cause of the discrepancy is much more complicated, because local physics is not applicable to this situation in principal. For this reason, if galaxies are relatively close to us-say, a few mega parsecs away-the speed of random motion makes the usual Hubble's law inapplicable; such galaxies can either move away or approach us. Hubble's law is fairly accurate only for distant galaxies, and then within certain limits-at very large distances of billions of light years (thousands of mega parsecs), the Hubble constant differs from the one accepted for closer galaxies.

8) However, Hubble's law is currently used as the simplest and most reliable way to determine the distances to galaxies and their clusters. This is the most important parameter describing the current rate of expansion of the Universe.

9) Extremely important to underline that the Hubble problem has two aspects matter evolution in space and the PV evolution in space.

\section{The Problem of Dark Matter: Dark Matter does not Exist. Analytical Investigation}

The problem of dark matter was considered by me before in the frame of the nonlocal description (see for example monographs [4] [6]). But here I intend to show that fundamental results of the observation astronomy can be obtained in the analytical form.

About forty years after Zwicky's initial observations Vera Rubin [33] [34] [35] [36], astronomer at the Department of Terrestrial Magnetism at the Carnegie Institution of Washington presented findings based on a new sensitive spectrograph that could measure the velocity curve of edge-on spiral galaxies to a greater degree of accuracy than had ever before been achieved. Together with Kent Ford, Rubin announced at a 1975 meeting of the American Astronomical Society the astonishing discovery that most stars in spiral galaxies orbit at roughly the same speed reflected schematically on Figure 9.

For example, the rotation curve of the type B corresponds to the galaxy NGC3198. The following extensive radio observations determined the detailed rotation curve of spiral disk galaxies to be flat (as the curve B), much beyond as seen in the optical band. Obviously the trivial balance between the gravitational and centrifugal forces leads to relation between orbital speed $V$ and galactocen- 
tric distance $r$ as $V^{2}=\gamma_{N} M / r$ beyond the physical extent of the galaxy of mass $M$ (the curve A). The obvious contradiction with the velocity curve $\mathrm{B}$ having a 'flat' appearance out to a large radius, was explained by introduction of a new physical essence-dark matter because for spherically symmetric case the hypothetical density distribution $\rho(r) \sim 1 / r^{2}$ leads to $V=$ const. The result of this activity is known undetectable dark matter which does not emit radiation, inferred solely from its gravitational effects. But it means that upwards of 50\% of the mass of galaxies was contained in the dark galactic halo.

Strict consideration leads to the system of the generalized hydrodynamic equations (GHE) (5.1)-(5.7) [2]-[7]. Regime B (Figure 9) cannot be obtained in the frame of local statistical physics in principal and authors of many papers introduce different approximations for additional "dark matter density" (as usual in Poisson equation) trying to find coincidence with data of observations. From the wrong position of local theories Poisson Equation (5.1) contains "dark matter density", continuity Equations (5.2) and (5.3) contain the "flux of dark matter density", motion Equations (5.4) and (5.5) include "dark energy", the energy Equations (5.6) and (5.7) has "the flux of dark energy" and so on to the "senior dark velocity moments". This entire situation is similar to the turbulent theories based on local statistical physics and empirical corrections for velocity moments.

The character features reflected on Figure 9 can be explained in the frame of Newtonian gravitation law and the non-local kinetic description [2]-[7]. Let us discuss mathematical and physical models involved into the mentioned consideration:

1) The typical galaxy contains the tremendous quantity of stars. As a result the hydrodynamic application for the investigation of the universe objects is typical in astrophysics. For example our home galaxy Milky Way contains over 200 billion stars including our Sun. The Milky Way has a diameter of 100,000 light years and belongs to the type of a barred spiral galaxy. The Milky Way has three main parts: a disk, in which the Solar System resides, a bulge at the core, and an all encompassing halo. This situation allows to apply the methods of statistical physics for the system description.

2) Peculiar features of the halo movement can be explained without new concepts like "dark matter". It is shown (see for example [6]) that the transformation

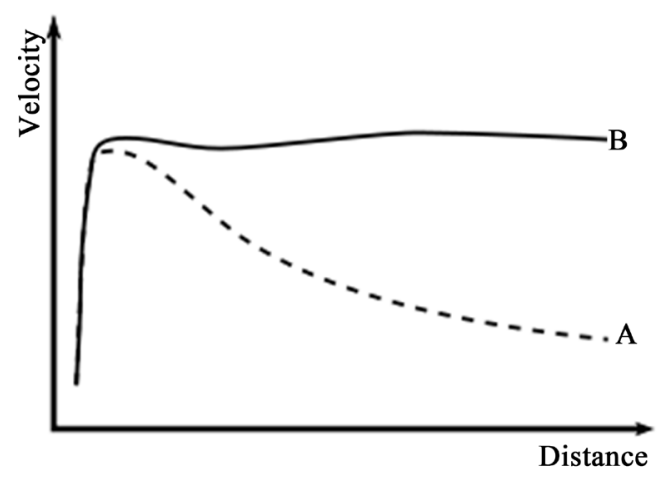

Figure 9. Rotation curve of a typical spiral galaxy: predicted (A) and observed (B). 
of the Kepler's regime into the flat rotation curves depends on the similarity parameter

$$
\tilde{\gamma}_{N}=\gamma_{N} \rho_{0} x_{0}^{2} / u_{0}^{2},
$$

where $\gamma_{N}$ is gravitational constant, $x_{0}$ and $u_{0}$ are the character length and the soliton velocity correspondingly. Parameter $\tilde{G}=\tilde{\gamma}_{N}$ plays the role of similarity criteria in traditional hydrodynamics. Important conclusions:

3) The obtained results demonstrate evolution the rotation curves from the Kepler regime (in the case of small $\tilde{G}$, like curve A on Figure 9) to observed orbital curves with the shelf (large $\tilde{G}$, like curve B on Figure 9) for typical spiral galaxies. Then parameter $\tilde{G}$ defines the transfer from the Kepler's to Rubin scenario.

4) The stars with planets (like Sun) correspond to gravitational soliton with small $\tilde{G}$ and therefore originate the Kepler rotation regime.

5) Important to underline that the shown transformation of the Kepler's regime into the flat rotation curves for different solitons explains the "mysterious" fact of the dark matter absence in the Sun vicinity.

I don't intend to repeat the corresponding calculations (see [6]). I intend to explain the obtained results in the frame of analytical investigation. We use the spherical coordinate system with the independent variables $r, \theta, \varphi$ (radial distance $r$, polar angle $\theta$ and azimuth angle $\varphi$ ):

Let us write down GHE (5.1)-(5.7) in spherical coordinate system for the one species system. We use continuity equation and motion equation written together for the physical system with radial symmetry

$$
\begin{aligned}
& \frac{\partial}{\partial t}\left\{\rho-\tau\left[\frac{\partial \rho}{\partial t}+\frac{1}{r^{2}} \frac{\partial\left(r^{2} \rho v_{0 r}\right)}{\partial r}\right]\right\} \\
& +\frac{1}{r^{2}} \frac{\partial}{\partial r}\left\{r^{2}\left[\rho v_{0 r}-\tau\left(\frac{1}{r^{2}} \frac{\partial\left(r^{2} \rho v_{0 r}^{2}\right)}{\partial r}-\rho g_{r}\right)\right]\right\}-\frac{1}{r^{2}} \frac{\partial}{\partial r}\left(\tau r^{2} \frac{\partial p}{\partial r}\right)=0 \\
& \frac{\partial}{\partial t}\left\{\rho v_{0 \varphi}-\tau\left[\frac{\partial}{\partial t}\left(\rho v_{0 \varphi}\right)+\frac{1}{r^{2}} \frac{\partial\left(r^{2} \rho v_{0 r} v_{0 \varphi}\right)}{\partial r}\right)\right\} \\
& +\frac{1}{r^{2}} \frac{\partial}{\partial r}\left\{r^{2}\left[\rho v_{0 r} v_{0 \varphi}-\tau\left(\frac{1}{r^{2}} \frac{\partial\left(r^{2} \rho v_{0 r}^{2} v_{0 \varphi}\right)}{\partial r}-g_{r} \rho v_{0 \varphi}\right)\right]\right\} \\
& -\frac{1}{r^{2}} \frac{\partial}{\partial r}\left(\tau r^{2} \frac{\partial\left(p v_{0 \varphi}\right)}{\partial r}\right)=0,
\end{aligned}
$$

where $v_{0 \varphi}$ is an orbital velocity. Two differential equations (8.2) and (8.3) are compatible even in the non-stationary case if

$$
v_{0 \varphi}=\text { const }
$$

for the arbitrary $\tau$.

Let us prolong the investigation of stationary case comparing the nonlocal 
continuity Equation (8.2) written in the form after integration

$$
\rho v_{0 r} v_{0 \varphi}-\tau\left(\frac{1}{r^{2}} v_{0 \varphi} \frac{\partial\left(r^{2} \rho v_{0 r}^{2}\right)}{\partial r}-g_{r} \rho v_{0 \varphi}+v_{0 \varphi} \frac{\partial p}{\partial r}\right)=0
$$

and the motion equation onto $\mathbf{e}_{\varphi}$-direction (8.3) we find

$$
\left(\rho v_{0 r}^{2}+p\right) \frac{\partial v_{0 \varphi}}{\partial r}=0
$$

or for satisfying (8.6) we should admit that $\frac{\partial v_{0 \varphi}}{\partial r}=0$ or

$$
v_{0 \varphi}=\text { const } .
$$

for all $r$. Extremely important that the relation (8.7) includes the Newton gravitation regime. Really, from the mathematical point of view the Newton's law has an asymptotic character if $r \rightarrow 0$ or $r \rightarrow \infty$

$$
F \sim \frac{1}{r^{2}}
$$

or

$$
v_{0 \varphi, N} \sim \frac{1}{r^{1 / 2}}
$$

After substitution (8.9) into (8.6) we have

$$
\left(\rho v_{0 r}^{2}+p\right) \frac{1}{r^{3 / 2}} \rightarrow 0
$$

if $r \rightarrow \infty$; total energy also tends to zero if $r \rightarrow \infty,\left(\rho v_{0 r}^{2}+p\right) \rightarrow 0$.

Let us investigate the Poisson Equation (5.1), which can be written in dimensionless form as ( $\tilde{G}$ is the dimensionless Newton gravitational constant $\tilde{\gamma}_{N}$ )

$$
\frac{\partial}{\partial \tilde{\mathbf{r}}} \cdot \tilde{\mathbf{g}}=-4 \pi \tilde{G}\left[\tilde{\rho}-\tilde{\tau}\left(\frac{\partial \tilde{\rho}}{\partial t}+\frac{\partial}{\partial \tilde{\mathbf{r}}} \cdot \tilde{\rho} \tilde{\mathbf{v}}_{0}\right)\right] .
$$

We suppose for the simplicity the stationary case with the radial symmetry. Then

$$
\frac{\partial}{\partial \tilde{r}}\left(\tilde{r}^{2} \tilde{g}_{r}\right)=-\tilde{G}\left[\tilde{\rho} \tilde{r}^{2}-\tilde{\tau} \frac{\partial\left(\tilde{r}^{2} \tilde{\rho} \tilde{v}_{0 r}\right)}{\partial \tilde{r}}\right]
$$

or after integration

$$
\tilde{r}^{2} \tilde{g}_{r}=-\tilde{G} \int_{0}^{\tilde{r}}\left[\tilde{\rho} \tilde{r}^{2}\right] \mathrm{d} \tilde{r}+\tilde{G} \int_{0}^{\tilde{r}}\left[\tilde{\tau} \frac{\partial\left(\tilde{r}^{2} \tilde{\rho} \tilde{v}_{0 r}\right)}{\partial \tilde{r}}\right] \mathrm{d} \tilde{r} .
$$

Let us suppose that nonlocal dimensionless parameter $\tilde{\tau}$ does not depend on $\tilde{r}$. In this case we find

$$
\tilde{r}^{2} \tilde{g}_{r}=-\tilde{G} \int_{0}^{\tilde{r}}\left[\tilde{\rho} \tilde{r}^{2}\right] \mathrm{d} \tilde{r}+\tilde{G} \tilde{\tau} \tilde{r}^{2} \tilde{\rho} \tilde{v}_{0 r}
$$

or 


$$
\tilde{r}^{2} \tilde{g}_{r}=-\tilde{G} \tilde{M}+\tilde{G} \tilde{\tau} \tilde{r}^{2} \tilde{\rho} \tilde{v}_{0 r},
$$

where $\tilde{M}$ is the dimensionless mass of the central body. As a result we obtain non-local gravitation law

$$
\tilde{g}_{r}=-\tilde{G} \frac{\tilde{M}}{\tilde{r}^{2}}+\tilde{\tau} \tilde{G} \tilde{\rho} \tilde{v}_{0 r}
$$

and only in the local case we reach the classical Newton law

$$
\tilde{g}_{r}=-\tilde{G} \frac{\tilde{M}}{\tilde{r}^{2}} .
$$

In the nonlocal case gravitational acceleration

$$
\tilde{g}_{r}=-\tilde{G}\left[\frac{\tilde{M}}{\tilde{r}^{2}}-\tilde{\tau} \tilde{\rho} \tilde{v}_{0 r}\right]
$$

turns into zero in a point $\tilde{r}_{c r}$ if

$$
\frac{\tilde{M}}{\tilde{r}_{c r}^{2}}=\tilde{\tau} \tilde{\rho} \tilde{v}_{0 r},
$$

where

$$
\tilde{r}_{c r}=\sqrt{\frac{\tilde{M}}{\tilde{\tau} \tilde{\rho} \tilde{v}_{0 r}}} .
$$

if $\tilde{v}_{0 r, c r}>0$. Then the well known relation should be modified

$$
m \tilde{g}_{r}=-\tilde{G} m\left[\frac{\tilde{M}}{\tilde{r}^{2}}-\tilde{\tau} \tilde{\rho} \tilde{v}_{0 r}\right]
$$

or the orbital velocity is equal

$$
V_{\text {orb }}=\sqrt{\tilde{G}\left[\frac{\tilde{M}}{\tilde{r}}-\tilde{\tau} \tilde{\rho} \tilde{v}_{0 r} \tilde{r}\right]} .
$$

The energy flux density can be negative $\tilde{\rho} \tilde{v}_{0 r}<0$ that is, can be $V_{\text {orb }}>V_{\text {orb,Newton }}$. This equality may mean the introduction of additional mass which in local physics as an "additional dark matter" is considered.

Then

$$
V_{\text {orb }}=\sqrt{\tilde{G} \frac{\tilde{M}}{\tilde{r}}\left[1-\frac{\tilde{\tau} \tilde{\rho} \tilde{v}_{0 r} \tilde{r}^{2}}{\tilde{M}}\right]} .
$$

For the bounded system $\tilde{\rho} \tilde{v}_{0 r}<0$; we have formally the additional (if you want "dark" mass)

$$
M_{\text {dark }}(\tilde{r})=\tilde{\tau} \tilde{\rho}\left|\tilde{v}_{0 r}\right| \tilde{r}^{2} .
$$

It means also that in the well known Soldner formulae the additional mass $M_{\text {dark }}(\tilde{r})$ should be inserted which leads to increasing effective mass $M$. For example (see [6])

$$
\delta_{N}=\frac{2 \gamma_{N}}{R_{\text {Sun }} c^{2}}\left(M_{\text {Sun }}+M_{\text {dark }}(\tilde{r})\right) .
$$

From the nonlocal Newton-Kepler relation (8.25) follows that anti-lensing ef- 
fect can take place. If the parameter $\tilde{\tau}$ is not dependent on $\tilde{r}$ the effective gravitational acceleration

$$
\tilde{g}_{r}=-\tilde{G}\left[\frac{\tilde{M}}{\tilde{r}^{2}}-\tilde{\tau} \tilde{\rho} \tilde{v}_{0 r}\right]
$$

can be positive, negative, or zero and only in the local case we reach the classical Newton law.

Let us compare (8.26) with the correction (8.27) following from the general relativity (GR)

$$
g_{r}=-G\left(\frac{M}{r^{2}}-\frac{4}{c^{2}} \frac{G M^{2}}{r^{3}}+\ldots\right) .
$$

As we see the corrections (8.26) and (8.27) have the same structure. Comparing formulas (8.26) and (8.27) allow defining the nonlocal parameter from the GR point of view.

Conclusions:

1) The kind of the halo rotation (8.7) corresponds to the Vera Rubin effect. In other words the orbital speed is constant for different radius.

2) This kind of the halo rotation corresponds to the Vera Rubin effect even in the presence of the mass transfer in the $r$ direction.

3) This analytical result does not depend on the $\tau$ choice.

4) The theory can be applied to the investigation of the ring rotation around the cosmic objects like Saturn. For example it means that the rotation inside of each Saturn rings corresponds to the Vera Rubin model. This effect can be verified by the direct observation or with the help of robotic spacecrafts.

5) The Newton law of gravitation for the statistical physical system should be modified.

Dark matter is artifact of local physics. Dark matter does not exist.

\section{Transport Processes in Physical Vacuum. Preliminary Remarks}

GHE have extremely important for astrophysics special cases when density $\rho \rightarrow 0$ (the initial stage of evolution of the Universe, the Big Bang) and when density $\rho \rightarrow \infty$ (evolution of the black hole). Both limiting cases have no physical or mathematical meaning in "classical" hydrodynamics. Thus, we have a unified statistical theory of dissipative structures, which has a hydrodynamic shape. We will, as already mentioned, refer to the corresponding system of equations as the fundamental equations of the unified theory (UT)

If the matter is absent, non-local evolution equations have nevertheless non- trivial solutions corresponding evolution of $\mathrm{PV}$ which description in time and $3 \mathrm{D}$ space on the level of quantum hydrodynamics demands only quantum pressure $p$, the self-consistent force $\mathbf{R}$ (acting on unit of the space volume) and velocity $\mathbf{v}_{0}$. The system of non local equations is written for the case when the usual matter is absent ( $\rho=0$ ); also radiation, gravitation (as well as other mass forces) and electromagnetic fields are absent. No reason to speak about special or general relativity 
in this situation, because these theories don't work in the described conditions.

We intend to consider from the position of the nonlocal physics the interaction of Physical Vacuum with matter.

We intend to consider the problem of the fundamental significance-is it possible to transfer the PV energy to matter? All results are obtained from the first principles of physics.

Another great problem of theoretical physics consists in asymmetry of matter and antimatter in the visible Universe.

What is the root of the problems from the theoretical point of view? The origin of difficulties consists in total Oversimplification following from principles of local physics and reflects the general shortcomings of the local kinetic transport theory based on the Boltzmann kinetic theory. Let us realize this program of investigation.

The process by which the inequality between matter and antimatter particles developed is called barogenesis. In modern physics, antimatter is defined as matter which is composed of the antiparticles of the corresponding particles of "ordinary" matter. Minuscule numbers of antiparticles can be found in natural processes like cosmic ray collisions and some types of radioactive decay. Only a tiny fraction of these have successfully been bound together in experiments to form anti-atoms. We consider this problem from the position of nonlocal physics.

With this aim we write down the GHE system of Equations (5.1)-(5.7) for the two component mixture of charged particles (without taking into account the component's internal energy) in the dimensionless form, where dimensionless symbols are marked by tildes. Summary of wave matter equations with the PV influence (subscripts " $\mathrm{i}$ " and " $\mathrm{e}$ " correspond to the positively and negatively charged components, subscript " $v$ " corresponds to PV).

We use the system of dimensional transport equations defining the interaction Matter-Physical Vacuum:

- Poisson equation

$$
\frac{\partial^{2} \psi}{\partial x^{2}}=-4 \pi e\left\{\left[n_{i}-\tau_{i}\left(\frac{\partial n_{i}}{\partial t}+\frac{\partial}{\partial x}\left(n_{i} u\right)\right)\right]-\left[n_{e}-\tau_{e}\left(\frac{\partial n_{e}}{\partial t}+\frac{\partial}{\partial x}\left(n_{e} u\right)\right)\right]\right\},
$$

- continuity equation for positive ion component

$$
\begin{aligned}
& \frac{\partial}{\partial t}\left\{\rho_{i}-\tau_{i}\left[\frac{\partial \rho_{i}}{\partial t}+\frac{\partial}{\partial x}\left(\rho_{i} u\right)\right]\right\} \\
& +\frac{\partial}{\partial x}\left\{\rho_{i} u-\tau_{i}\left[\frac{\partial}{\partial t}\left(\rho_{i} u\right)+\frac{\partial}{\partial x}\left(\rho_{i} u^{2}\right)+\frac{\partial p_{i}}{\partial x}-\rho_{i} F_{i, \text { eff }}\right]\right\}=0,
\end{aligned}
$$

- continuity equation for electron component

$$
\begin{aligned}
& \frac{\partial}{\partial t}\left\{\rho_{e}-\tau_{e}\left[\frac{\partial \rho_{e}}{\partial t}+\frac{\partial}{\partial x}\left(\rho_{e} u\right)\right]\right\} \\
& +\frac{\partial}{\partial x}\left\{\rho_{e} u-\tau_{e}\left[\frac{\partial}{\partial t}\left(\rho_{e} u\right)+\frac{\partial}{\partial x}\left(\rho_{e} u^{2}\right)+\frac{\partial p_{e}}{\partial x}-\rho_{e} F_{e, e f f}\right]\right\}=0,
\end{aligned}
$$

- momentum equation 


$$
\begin{aligned}
& \frac{\partial}{\partial t}\left\{\rho u-\tau_{i}\left[\frac{\partial}{\partial t}\left(\rho_{i} u\right)+\frac{\partial}{\partial x}\left(p_{i}+\rho_{i} u^{2}\right)-\rho_{i} F_{i, e f f}\right]-\tau_{e}\left[\frac{\partial}{\partial t}\left(\rho_{e} u\right)+\frac{\partial}{\partial x}\left(p_{e}+\rho_{e} u^{2}\right)-\rho_{e} F_{e, e f f}\right]\right\} \\
& -\rho_{i} F_{i, e f f}-\rho_{e} F_{e, e f f}+F_{i, e f f} \tau_{i}\left(\frac{\partial \rho_{i}}{\partial t}+\frac{\partial}{\partial x}\left(\rho_{i} u\right)\right)+F_{e, e f f} \tau_{e}\left(\frac{\partial \rho_{e}}{\partial t}+\frac{\partial}{\partial x}\left(\rho_{e} u\right)\right) \\
& +\frac{\partial}{\partial x}\left\{\rho u^{2}+p-\tau_{i}\left[\frac{\partial}{\partial t}\left(\rho_{i} u^{2}+p_{i}\right)+\frac{\partial}{\partial x}\left(\rho_{i} u^{3}+3 p_{i} u\right)-2 \rho_{i} u F_{i, e f f}\right]\right. \\
& \left.-\tau_{e}\left[\frac{\partial}{\partial t}\left(\rho_{e} u^{2}+p_{e}\right)+\frac{\partial}{\partial x}\left(\rho_{e} u^{3}+3 p_{e} u\right)-2 \rho_{e} u F_{e, e f f}\right]\right\}=0 ;
\end{aligned}
$$

- energy equation for positive ion component

$$
\begin{aligned}
& \frac{\partial}{\partial t}\left\{\rho_{i} u^{2}+3 p_{i}-\tau_{i}\left[\frac{\partial}{\partial t}\left(\rho_{i} u^{2}+3 p_{i}\right)+\frac{\partial}{\partial x}\left(\rho_{i} u^{3}+5 p_{i} u\right)-2 \rho_{i} F_{i, e f f} u\right]\right\} \\
& +\frac{\partial}{\partial x}\left\{\rho_{i} u^{3}+5 p_{i} u-\tau_{i}\left[\frac{\partial}{\partial t}\left(\rho_{i} u^{3}+5 p_{i} u\right)\right.\right. \\
& \left.\left.+\frac{\partial}{\partial x}\left(\rho_{i} u^{4}+8 p_{i} u^{2}+5 \frac{p_{i}^{2}}{\rho_{i}}\right)-F_{i, \text { eff }}\left(3 \rho_{i} u^{2}+5 p_{i}\right)\right]\right\} \\
& -2 u \rho_{i} F_{i, \text { eff }}+2 \tau_{i} F_{i, \text { eff }}\left[\frac{\partial}{\partial t}\left(\rho_{i} u\right)+\frac{\partial}{\partial x}\left(\rho_{i} u^{2}+p_{i}\right)-\rho_{i} F_{i, \text { eff }}\right]=-\frac{p_{i}-p_{e}}{\tau_{e i}},
\end{aligned}
$$

- energy equation for electron component

$$
\begin{aligned}
& \frac{\partial}{\partial t}\left\{\rho_{e} u^{2}+3 p_{e}-\tau_{e}\left[\frac{\partial}{\partial t}\left(\rho_{e} u^{2}+3 p_{e}\right)+\frac{\partial}{\partial x}\left(\rho_{e} u^{3}+5 p_{e} u\right)-2 \rho_{e} F_{e, e f f} u\right]\right\} \\
& +\frac{\partial}{\partial x}\left\{\rho_{e} u^{3}+5 p_{e} u-\tau_{e}\left[\frac{\partial}{\partial t}\left(\rho_{e} u^{3}+5 p_{e} u\right)\right.\right. \\
& \left.\left.+\frac{\partial}{\partial x}\left(\rho_{e} u^{4}+8 p_{e} u^{2}+5 \frac{p_{e}^{2}}{\rho_{e}}\right)-F_{e, e f f}\left(3 \rho_{e} u^{2}+5 p_{e}\right)\right]\right\} \\
& -2 u \rho_{e} F_{e, e f f}+2 \tau_{e} F_{e, e f f}\left[\frac{\partial}{\partial t}\left(\rho_{e} u\right)+\frac{\partial}{\partial x}\left(\rho_{e} u^{2}+p_{e}\right)-\rho_{e} F_{e, e f f}\right]=-\frac{p_{e}-p_{i}}{\tau_{e i}},
\end{aligned}
$$

where $u$ is translational velocity of the quantum object, $\psi$-scalar potential, $n_{i}$ and $n_{e}$ are the number density of the charged species, $F_{i, \text { eff }}$ and $F_{e, \text { eff }}$ are the forces acting on the unit mass of ion and electron with taking into account the PV influence.

For the $\tau_{i}$ and $\tau_{e}$ approximations, the relations are used in the forms

$$
\tau_{i}=\frac{\hbar}{m_{i} u^{2}}, \tau_{e}=\frac{\hbar}{m_{e} u^{2}} .
$$

We begin with introduction the scales for velocity

$$
[u]=u_{0}
$$

and for coordinate $x \frac{\hbar}{m_{e} u_{0}}=x_{0}$, for $\psi \rightarrow \psi_{0}$, for $n \rightarrow n_{0}$.

Generalized Poisson Equation (9.1) now is written as

$$
\frac{\partial^{2} \psi}{\partial x^{2}}=-4 \pi e\left\{\left[n_{i}-\tau_{i}\left(\frac{\partial n_{i}}{\partial t}+\frac{\partial}{\partial x}\left(n_{i} u\right)\right)\right]-\left[n_{e}-\tau_{e}\left(\frac{\partial n_{e}}{\partial t}+\frac{\partial}{\partial x}\left(n_{e} u\right)\right)\right]\right\},
$$


or

$$
\begin{aligned}
\psi_{0} \frac{\partial^{2} \tilde{\psi}}{\partial \tilde{x}^{2}}= & -4 \pi e \rho_{0} x_{0}^{2}\left\{\left[\frac{1}{m_{i}} \tilde{\rho}_{i}-\tau_{i} \frac{1}{m_{i}}\left(\frac{\partial \tilde{\rho}_{i}}{\partial t}+\frac{u_{0}}{x_{0}} \frac{\partial}{\partial \tilde{x}}\left(\tilde{\rho}_{i} \tilde{u}\right)\right)\right]\right. \\
& \left.-\left[\frac{1}{m_{e}} \rho_{e}-\tau_{e} \frac{1}{m_{e}}\left(\frac{\partial \tilde{\rho}_{e}}{\partial t}+\frac{u_{0}}{x_{0}} \frac{\partial}{\partial \tilde{x}}\left(\tilde{\rho}_{e} \tilde{u}\right)\right)\right]\right\} .
\end{aligned}
$$

If the potential scale $\psi_{0}$ and the density scale $\rho_{0}$ are chosen as

$$
\begin{gathered}
\psi_{0}=\frac{m_{e}}{e} u_{0}^{2}, \\
\rho_{0}=\frac{m_{e}^{4}}{4 \pi \hbar^{2} e^{2}} u_{0}^{4} .
\end{gathered}
$$

we find

$$
\begin{aligned}
\frac{\partial^{2} \tilde{\psi}}{\partial \tilde{x}^{2}}= & -4 \pi x_{0}^{2} \frac{m_{e}^{3}}{4 \pi \hbar^{2}} u_{0}^{2}\left\{\left[\frac{1}{m_{i}} \tilde{\rho}_{i}-\tau_{i} \frac{1}{m_{i}}\left(\frac{\partial \tilde{\rho}_{i}}{\partial t}+\frac{u_{0}}{x_{0}} \frac{\partial}{\partial \tilde{x}}\left(\tilde{\rho}_{i} \tilde{u}\right)\right)\right]\right. \\
& \left.-\left[\frac{1}{m_{e}} \tilde{\rho}_{e}-\tau_{e} \frac{1}{m_{e}}\left(\frac{\partial \tilde{\rho}_{e}}{\partial t}+\frac{u_{0}}{x_{0}} \frac{\partial}{\partial \tilde{x}}\left(\tilde{\rho}_{e} \tilde{u}\right)\right)\right]\right\} .
\end{aligned}
$$

Let us introduce the wave variable for the plane flow

$$
\xi=x-C t
$$

using the scales

$$
\begin{gathered}
\frac{\hbar}{m_{e} u_{0}}=x_{0}, \\
t_{0}=\frac{x_{0}}{C}
\end{gathered}
$$

we reach the dimensionless form of the independent variable (9.14)

$$
\tilde{\xi}=\tilde{x}-\tilde{t} .
$$

Using (9.7) and (9.15) one obtains

$$
\begin{aligned}
\frac{\partial^{2} \tilde{\psi}}{\partial \tilde{x}^{2}}= & -m_{e}\left\{\left[\frac{1}{m_{i}} \tilde{\rho}_{i}-\frac{\hbar}{u^{2} m_{i}^{2}}\left(-\frac{C}{x_{0}} \frac{\partial \tilde{\rho}_{i}}{\partial \tilde{\xi}}+\frac{u_{0}}{x_{0}} \frac{\partial}{\partial \tilde{\xi}}\left(\tilde{\rho}_{i} \tilde{u}\right)\right)\right]\right. \\
& {\left.\left[-\frac{1}{m_{e}} \tilde{\rho}_{e}-\frac{\hbar}{u^{2} m_{e}^{2}}\left(-\frac{C}{x_{0}} \frac{\partial \tilde{\rho}_{e}}{\partial \tilde{\xi}}+\frac{u_{0}}{x_{0}} \frac{\partial}{\partial \tilde{\xi}}\left(\tilde{\rho}_{e} \tilde{u}\right)\right)\right]\right\} }
\end{aligned}
$$

Let be

$$
C=u_{0} .
$$

We have finally from (9.15)-(9.18)

$$
\begin{aligned}
\frac{\partial^{2} \tilde{\psi}}{\partial \tilde{\xi}^{2}}= & -\left\{\frac{m_{e}}{m_{i}}\left[\tilde{\rho}_{i}-\frac{1}{\tilde{u}^{2}} \frac{m_{e}}{m_{i}}\left(-\frac{\partial \tilde{\rho}_{i}}{\partial \tilde{\xi}}+\frac{\partial}{\partial \tilde{\xi}}\left(\tilde{\rho}_{i} \tilde{u}\right)\right)\right]\right. \\
& \left.-\left[\tilde{\rho}_{e}-\frac{1}{\tilde{u}^{2}}\left(-\frac{\partial \tilde{\rho}_{e}}{\partial \tilde{\xi}}+\frac{\partial}{\partial \tilde{\xi}}\left(\tilde{\rho}_{e} \tilde{u}\right)\right)\right]\right\} .
\end{aligned}
$$


We transform now the continuity equations for $i$ and $e$ components. One obtains for the positive charged $i$ species

$$
\begin{aligned}
& \frac{\partial}{\partial t}\left\{\rho_{i}-\tau_{i}\left[\frac{\partial \rho_{i}}{\partial t}+\frac{\partial}{\partial x}\left(\rho_{i} u\right)\right]\right\} \\
& +\frac{\partial}{\partial x}\left\{\rho_{i} u-\tau_{i}\left[\frac{\partial}{\partial t}\left(\rho_{i} u\right)+\frac{\partial}{\partial x}\left(\rho_{i} u^{2}\right)+\frac{\partial p_{i}}{\partial x}-\rho_{i} F_{i, \text { eff }}\right]\right\}=0 .
\end{aligned}
$$

In the moving coordinate system all dependent hydrodynamic values are functions of $(\xi, t)$. We investigate the possibility of the quantum object formation of the soliton type. For this solution there is no explicit dependence on time for coordinate system moving with the phase velocity $u_{0}$. Then using the scale relation

$$
\frac{1}{t_{0}}=\frac{u_{0}}{x_{0}}
$$

and (9.7) we have

$$
\begin{aligned}
& -\frac{\partial \tilde{\rho}_{i}}{\partial \tilde{\xi}}+\frac{\partial}{\partial \tilde{\xi}}\left(\tilde{\rho}_{i} \tilde{u}\right)+\frac{u_{0}}{x_{0}} \frac{\partial}{\partial \tilde{\xi}}\left\{\frac{\hbar}{m_{i} u^{2}}\left[-\frac{\partial \tilde{\rho}_{i}}{\partial \tilde{\xi}}+\frac{\partial}{\partial \tilde{\xi}}\left(\tilde{\rho}_{i} \tilde{u}\right)\right]\right\} \\
& -\frac{u_{0}}{x_{0}} \frac{\partial}{\partial \tilde{\xi}}\left\{\frac{\hbar}{m_{i} u^{2}}\left[-\frac{\partial}{\partial \tilde{\xi}}\left(\tilde{\rho}_{i} \tilde{u}\right)+\frac{\partial}{\partial \tilde{\xi}}\left(\tilde{\rho}_{i} \tilde{u}^{2}\right)+\frac{p_{0}}{\rho_{0} u_{0}^{2}} \frac{\partial \tilde{p}_{i}}{\partial \tilde{\xi}}-\tilde{\rho}_{i} F_{i, e f f} \frac{x_{0}}{u_{0}^{2}}\right]\right\}=0
\end{aligned}
$$

Let us introduce the scale

$$
F_{0} \rightarrow \frac{u_{0}^{2}}{x_{0}}
$$

the scale

$$
p \rightarrow p_{0}=\rho_{0} u_{0}^{2} .
$$

and transform (9.23)

$$
\begin{aligned}
& \frac{\partial \tilde{\rho}_{i}}{\partial \tilde{\xi}}-\frac{\partial}{\partial \tilde{\xi}}\left(\tilde{\rho}_{i} \tilde{u}\right)-\frac{m_{e}}{m_{i}} \frac{\partial}{\partial \tilde{\xi}}\left\{\frac{1}{\tilde{u}^{2}}\left[-\frac{\partial \tilde{\rho}_{i}}{\partial \tilde{\xi}}+\frac{\partial}{\partial \tilde{\xi}}\left(\tilde{\rho}_{i} \tilde{u}\right)\right]\right\} \\
& +\frac{m_{e}}{m_{i}} \frac{\partial}{\partial \tilde{\xi}}\left\{\frac{1}{\tilde{u}^{2}}\left[-\frac{\partial}{\partial \tilde{\xi}}\left(\tilde{\rho}_{i} \tilde{u}\right)+\frac{\partial}{\partial \tilde{\xi}}\left(\tilde{\rho}_{i} \tilde{u}^{2}\right)+\frac{\partial \tilde{p}_{i}}{\partial \tilde{\xi}}-\tilde{\rho}_{i} \tilde{F}_{i, e f f}\right]\right\}=0 .
\end{aligned}
$$

The forces of the electric origin have the form

$$
\begin{gathered}
\rho_{i} F_{i}=-\frac{u_{0}^{2}}{x_{0}} \rho_{0} \frac{m_{e}}{m_{i}} \frac{\partial \tilde{\psi}}{\partial \tilde{\xi}} \tilde{\rho}_{i}, \\
\rho_{e} F_{e}=\frac{u_{0}^{2}}{x_{0}} \rho_{0} \frac{\partial \tilde{\psi}}{\partial \tilde{\xi}} \tilde{\rho}_{e},
\end{gathered}
$$

but we should add the volume forces of the PV origin. We find

$$
\begin{aligned}
& \frac{\partial \tilde{\rho}_{i}}{\partial \tilde{\xi}}-\frac{\partial}{\partial \tilde{\xi}}\left(\tilde{\rho}_{i} \tilde{u}\right) \\
& +\frac{m_{e}}{m_{i}} \frac{\partial}{\partial \tilde{\xi}}\left\{\frac{1}{\tilde{u}^{2}}\left[\frac{\partial}{\partial \tilde{\xi}}\left(\tilde{p}_{i}+\tilde{\rho}_{i}+\tilde{\rho}_{i} \tilde{u}^{2}-2 \tilde{\rho}_{i} \tilde{u}\right)-\tilde{\rho}_{i} \tilde{F}_{i}-\frac{1}{1+\frac{\tilde{\rho}_{e}}{\tilde{\rho}_{i}} \frac{m_{i}}{m_{e}}} \frac{\partial \tilde{p}_{v}}{\partial \tilde{\xi}}\right]\right\}=0
\end{aligned}
$$


where

$$
\tilde{F}_{i}=-\frac{m_{e}}{m_{i}} \frac{\partial \tilde{\psi}}{\partial \tilde{\xi}} .
$$

Let us realize transformation of the continuity equation for $e$-species to the dimensionless form

$$
\begin{aligned}
& \frac{\partial \rho_{e}}{\partial t}+\frac{\partial}{\partial x}\left(\rho_{e} u\right)-\frac{\partial}{\partial t}\left\{\tau_{e}\left[\frac{\partial \rho_{e}}{\partial t}+\frac{\partial}{\partial x}\left(\rho_{e} u\right)\right]\right\} \\
& -\frac{\partial}{\partial x}\left\{\tau_{e}\left[\frac{\partial}{\partial t}\left(\rho_{e} u\right)+\frac{\partial}{\partial x}\left(\rho_{e} u^{2}\right)+\frac{\partial p_{e}}{\partial x}-\rho_{e} F_{e, e f f}\right]\right\}=0,
\end{aligned}
$$

Using the relations $\frac{1}{t_{0}}=\frac{u_{0}}{x_{0}}$ and $\tau_{e}=\frac{\hbar}{m_{e} u^{2}}$ one obtains

$$
\begin{aligned}
& -\frac{\partial \tilde{\rho}_{e}}{\partial \tilde{\xi}}+\frac{\partial}{\partial \tilde{\xi}}\left(\tilde{\rho}_{e} \tilde{u}\right)+\frac{u_{0}}{x_{0}} \frac{\partial}{\partial \tilde{\xi}}\left\{\frac{\hbar}{m_{e} u^{2}}\left[-\frac{\partial \tilde{\rho}_{e}}{\partial \tilde{\xi}}+\frac{\partial}{\partial \tilde{\xi}}\left(\tilde{\rho}_{e} \tilde{u}\right)\right]\right\} \\
& -\frac{u_{0}}{x_{0}} \frac{\partial}{\partial \tilde{\xi}}\left\{\frac{\hbar}{m_{e} u^{2}}\left[-\frac{\partial}{\partial \tilde{\xi}}\left(\tilde{\rho}_{e} \tilde{u}\right)+\frac{\partial}{\partial \tilde{\xi}}\left(\tilde{\rho}_{e} \tilde{u}^{2}\right)+\frac{p_{0}}{\rho_{0} u_{0}^{2}} \frac{\partial \tilde{p}_{e}}{\partial \tilde{\xi}}-\tilde{\rho}_{e} F_{e, e f f} \frac{x_{0}}{u_{0}^{2}}\right]\right\}=0
\end{aligned}
$$

Let us introduce the scale $F_{0} \rightarrow \frac{u_{0}^{2}}{x_{0}}$ and the scale $p \rightarrow p_{0}=\rho_{0} u_{0}^{2}$; I remind that $v_{q u}=\frac{h}{m_{e}}$ is introduced by me quantum kinematic viscosity and $x_{0}=\frac{\hbar}{m_{e} u_{0}}=\frac{v_{q u}}{2 \pi u_{0}}$. We have

$$
\begin{aligned}
& \frac{\partial \tilde{\rho}_{e}}{\partial \tilde{\xi}}-\frac{\partial}{\partial \tilde{\xi}}\left(\tilde{\rho}_{e} \tilde{u}\right)-\frac{\partial}{\partial \tilde{\xi}}\left\{\frac{1}{\tilde{u}^{2}}\left[-\frac{\partial \tilde{\rho}_{e}}{\partial \tilde{\xi}}+\frac{\partial}{\partial \tilde{\xi}}\left(\tilde{\rho}_{e} \tilde{u}\right)\right]\right\} \\
& +\frac{\partial}{\partial \tilde{\xi}}\left\{\frac{1}{\tilde{u}^{2}}\left[\frac{\partial}{\partial \tilde{\xi}}\left(\tilde{p}_{e}+\tilde{\rho}_{e}+\tilde{\rho}_{e} \tilde{u}^{2}-2 \tilde{\rho}_{e} \tilde{u}\right)-\tilde{\rho}_{e} \tilde{F}_{e}-\frac{1}{1+\frac{\tilde{\rho}_{i}}{\tilde{\rho}_{e}} \frac{m_{e}}{m_{i}}} \frac{\partial \tilde{p}_{v}}{\partial \tilde{\xi}}\right]\right\}=0,
\end{aligned}
$$

where

$$
\tilde{\rho}_{e} \tilde{F}_{e}=\frac{\partial \tilde{\psi}}{\partial \tilde{\xi}} \tilde{\rho}_{e}
$$

We transform now the motion equation;

$$
\begin{aligned}
& \frac{\partial}{\partial t}\left\{\rho u-\tau_{i}\left[\frac{\partial}{\partial t}\left(\rho_{i} u\right)+\frac{\partial}{\partial x}\left(p_{i}+\rho_{i} u^{2}\right)-\rho_{i} F_{i, e f f}\right]\right. \\
& \left.-\tau_{e}\left[\frac{\partial}{\partial t}\left(\rho_{e} u\right)+\frac{\partial}{\partial x}\left(p_{e}+\rho_{e} u^{2}\right)-\rho_{e} F_{e, e f f}\right]\right\} \\
& -\rho_{i} F_{i, \text { eff }}-\rho_{e} F_{e, \text { eff }}+F_{i, e f f} \tau_{i}\left(\frac{\partial \rho_{i}}{\partial t}+\frac{\partial}{\partial x}\left(\rho_{i} u\right)\right)+F_{e, e f f} \tau_{e}\left(\frac{\partial \rho_{e}}{\partial t}+\frac{\partial}{\partial x}\left(\rho_{e} u\right)\right) \\
& +\frac{\partial}{\partial x}\left\{\rho u^{2}+p-\tau_{i}\left[\frac{\partial}{\partial t}\left(\rho_{i} u^{2}+p_{i}\right)+\frac{\partial}{\partial x}\left(\rho_{i} u^{3}+3 p_{i} u\right)-2 \rho_{i} u F_{i, e f f}\right]\right. \\
& \left.-\tau_{e}\left[\frac{\partial}{\partial t}\left(\rho_{e} u^{2}+p_{e}\right)+\frac{\partial}{\partial x}\left(\rho_{e} u^{3}+3 p_{e} u\right)-2 \rho_{e} u F_{e, e f f}\right]\right\}=0 ;
\end{aligned}
$$




\section{We find}

$-\frac{\partial}{\partial \tilde{\xi}}\left\{\tilde{\rho} \tilde{u}-\tau_{i} \frac{u_{0}}{x_{0}}\left[-\frac{\partial}{\partial \tilde{\xi}}\left(\tilde{\rho}_{i} \tilde{u}\right)+\frac{\partial}{\partial \tilde{\xi}}\left(\tilde{p}_{i}+\tilde{\rho}_{i} \tilde{u}^{2}\right)-\tilde{\rho}_{i} F_{i, e f f} \frac{x_{0}}{u_{0}^{2}}\right]\right.$

$\left.-\tau_{e} \frac{u_{0}}{x_{0}}\left[-\frac{\partial}{\partial \tilde{\xi}}\left(\tilde{\rho}_{e} \tilde{u}\right)+\frac{\partial}{\partial \tilde{\xi}}\left(\tilde{p}_{e}+\tilde{\rho}_{e} \tilde{u}^{2}\right)-\tilde{\rho}_{e} F_{e, e f f} \frac{x_{0}}{u_{0}^{2}}\right]\right\}$

$-\frac{x_{0}}{u_{0}^{2}}\left(\tilde{\rho}_{i} F_{i, e f f}+\tilde{\rho}_{e} F_{e, e f f}\right)+F_{i, e f f} \tau_{i} \frac{1}{u_{0}}\left(-\frac{\partial \tilde{\rho}_{i}}{\partial \tilde{\xi}}+\frac{\partial}{\partial \tilde{\xi}}\left(\tilde{\rho}_{i} \tilde{u}\right)\right)$

$+F_{e, e f f} \tau_{e} \frac{1}{u_{0}}\left(-\frac{\partial \tilde{\rho}_{e}}{\partial \tilde{\xi}}+\frac{\partial}{\partial \tilde{\xi}}\left(\tilde{\rho}_{e} \tilde{u}\right)\right)$

$+\frac{\partial}{\partial \tilde{\xi}}\left\{\tilde{\rho} \tilde{u}^{2}+\tilde{p}-\tau_{i} \frac{u_{0}}{x_{0}}\left[-\frac{\partial}{\partial \tilde{\xi}}\left(\tilde{p}_{i}+\tilde{\rho}_{i} \tilde{u}^{2}\right)+\frac{\partial}{\partial \tilde{\xi}}\left(\tilde{\rho}_{i} \tilde{u}^{3}+3 \tilde{p}_{i} \tilde{u}\right)-2 \tilde{u} \tilde{\rho}_{i} F_{i, e f f} \frac{x_{0}}{u_{0}^{2}}\right]\right.$

$\left.-\tau_{e} \frac{u_{0}}{x_{0}}\left[-\frac{\partial}{\partial \tilde{\xi}}\left(\tilde{p}_{e}+\tilde{\rho}_{e} \tilde{u}^{2}\right)+\frac{\partial}{\partial \tilde{\xi}}\left(\tilde{\rho}_{e} \tilde{u}^{3}+3 \tilde{p}_{e} \tilde{u}\right)-2 \tilde{u} \tilde{\rho}_{e} F_{e, e f f} \frac{x_{0}}{u_{0}^{2}}\right]\right\}=0$

Using the relations $\tau_{i}=\frac{\hbar}{m_{i} u^{2}}, \tau_{i}=\frac{\hbar}{m_{i} u^{2}}, \frac{\hbar}{m_{e} u_{0}}=x_{0}$ and $F_{0}=\frac{u_{0}^{2}}{x_{0}}$ we have

$$
\begin{aligned}
& -\frac{\partial}{\partial \tilde{\xi}}\left\{\tilde{\rho} \tilde{u}-\frac{1}{\tilde{u}^{2}} \frac{m_{e}}{m_{i}}\left[-\frac{\partial}{\partial \tilde{\xi}}\left(\tilde{\rho}_{i} \tilde{u}\right)+\frac{\partial}{\partial \tilde{\xi}}\left(\tilde{p}_{i}+\tilde{\rho}_{i} \tilde{u}^{2}\right)-\tilde{\rho}_{i} \tilde{F}_{i, e f f}\right]\right. \\
& \left.-\frac{1}{\tilde{u}^{2}}\left[-\frac{\partial}{\partial \tilde{\xi}}\left(\tilde{\rho}_{e} \tilde{u}\right)+\frac{\partial}{\partial \tilde{\xi}}\left(\tilde{p}_{e}+\tilde{\rho}_{e} \tilde{u}^{2}\right)-\tilde{\rho}_{e} \tilde{F}_{e, e f f}\right]\right\} \\
& -\left(\tilde{\rho}_{i} \tilde{F}_{i, e f f}+\tilde{\rho}_{e} \tilde{F}_{e, e f f}\right)+\tilde{F}_{i, e f f} \frac{u_{0}^{2}}{x_{0}} \frac{\hbar}{m_{i} u^{2}} \frac{1}{u_{0}}\left(-\frac{\partial \tilde{\rho}_{i}}{\partial \tilde{\xi}}+\frac{\partial}{\partial \tilde{\xi}^{\prime}}\left(\tilde{\rho}_{i} \tilde{u}\right)\right) \\
& +\tilde{F}_{e, e f f} \frac{u_{0}^{2}}{x_{0}} \frac{\hbar}{m_{e} u^{2}} \frac{1}{u_{0}}\left(-\frac{\partial \tilde{\rho}_{e}}{\partial \tilde{\xi}}+\frac{\partial}{\partial \tilde{\xi}}\left(\tilde{\rho}_{e} \tilde{u}\right)\right) \\
& +\frac{\partial}{\partial \tilde{\xi}}\left\{\tilde{\rho} \tilde{u}^{2}+\tilde{p}-\frac{1}{\tilde{u}^{2}} \frac{m_{e}}{m_{i}}\left[-\frac{\partial}{\partial \tilde{\xi}}\left(\tilde{p}_{i}+\tilde{\rho}_{i} \tilde{u}^{2}\right)+\frac{\partial}{\partial \tilde{\xi}}\left(\tilde{\rho}_{i} \tilde{u}^{3}+3 \tilde{p}_{i} \tilde{u}\right)-2 \tilde{u} \tilde{\rho}_{i} \tilde{F}_{i, e f f}\right]\right. \\
& \left.-\frac{1}{\tilde{u}^{2}}\left[-\frac{\partial}{\partial \tilde{\xi}}\left(\tilde{p}_{e}+\tilde{\rho}_{e} \tilde{u}^{2}\right)+\frac{\partial}{\partial \tilde{\xi}}\left(\tilde{\rho}_{e} \tilde{u}^{3}+3 \tilde{p}_{e} \tilde{u}\right)-2 \tilde{u} \tilde{\rho}_{e} \tilde{F}_{e, e f f}\right]\right\}=0 ;
\end{aligned}
$$

or

$$
\begin{aligned}
& \frac{\partial}{\partial \tilde{\xi}}\left\{\left(\tilde{\rho}_{i}+\tilde{\rho}_{e}\right) \tilde{u}^{2}+\left(\tilde{p}_{i}+\tilde{p}_{e}\right)-\left(\tilde{\rho}_{i}+\tilde{\rho}_{e}\right) \tilde{u}\right\} \\
& +\frac{\partial}{\partial \tilde{\xi}}\left\{\frac{1}{\tilde{u}^{2}} \frac{m_{e}}{m_{i}}\left[\frac{\partial}{\partial \tilde{\xi}}\left(2 \tilde{p}_{i}+2 \tilde{\rho}_{i} \tilde{u}^{2}-\tilde{\rho}_{i} \tilde{u}-\tilde{\rho}_{i} \tilde{u}^{3}-3 \tilde{p}_{i} \tilde{u}\right)-\tilde{\rho}_{i} \tilde{F}_{i}-\frac{1}{1+\frac{\tilde{\rho}_{e}}{\tilde{\rho}_{i}} \frac{m_{i}}{m_{e}}} \frac{\partial \tilde{p}_{v}}{\partial \tilde{\xi}}\right]\right. \\
& \left.+\frac{1}{\tilde{u}^{2}}\left[\frac{\partial}{\partial \tilde{\xi}}\left(2 \tilde{p}_{e}+2 \tilde{\rho}_{e} \tilde{u}^{2}-\tilde{\rho}_{e} \tilde{u}-\tilde{\rho}_{e} \tilde{u}^{3}-3 \tilde{p}_{e} \tilde{u}\right)-\tilde{\rho}_{e} \tilde{F}_{e}-\frac{\tilde{\rho}_{e}}{\tilde{\rho}_{e}+\tilde{\rho}_{i} \frac{m_{e}}{m_{i}}} \frac{\partial \tilde{p}_{v}}{\partial \tilde{\xi}}\right]\right\} \\
& -\left(\tilde{\rho}_{i} \tilde{F}_{i}+\tilde{\rho}_{e} \tilde{F}_{e}\right)-\frac{\tilde{\rho}_{i}}{\tilde{\rho}_{i}+\tilde{\rho}_{e} \frac{m_{i}}{m_{e}}} \frac{\partial \tilde{p}_{v}}{\partial \tilde{\xi}}-\frac{\tilde{\rho}_{e}}{\tilde{\rho}_{e}+\tilde{\rho}_{i} \frac{m_{e}}{m_{i}}} \frac{\partial \tilde{p}_{v}}{\partial \tilde{\xi}}
\end{aligned}
$$




$$
\begin{aligned}
& +\left(\tilde{F}_{i}+\frac{1}{\rho_{i}+\tilde{\rho}_{e} \frac{m_{i}}{m_{e}}} \frac{\partial \tilde{p}_{v}}{\partial \tilde{\xi}}\right) \frac{1}{\tilde{u}^{2}} \frac{m_{e}}{m_{i}} \frac{\partial}{\partial \tilde{\xi}}\left[\tilde{\rho}_{i}(\tilde{u}-1)\right] \\
& +\left(\tilde{F}_{e}+\frac{1}{\tilde{\rho}_{e}+\tilde{\rho}_{i} \frac{m_{e}}{m_{i}}} \frac{\partial \tilde{p}_{v}}{\partial \tilde{\xi}}\right) \frac{1}{\tilde{u}^{2}} \frac{\partial}{\partial \tilde{\xi}}\left[\tilde{\rho}_{e}(\tilde{u}-1)\right] \\
& +2 \frac{\partial}{\partial \tilde{\xi}}\left\{\frac{1}{\tilde{u}}\left[\tilde{\rho}_{e} \tilde{F}_{e}+\frac{\tilde{\rho}_{e}}{\tilde{\rho}_{e}+\tilde{\rho}_{i} \frac{m_{e}}{m_{i}}} \frac{\partial \tilde{p}_{v}}{\partial \tilde{\xi}}\right]\right\}+2 \frac{m_{e}}{m_{i}} \frac{\partial}{\partial \tilde{\xi}}\left(\frac{1}{\tilde{u}}\left[\tilde{\rho}_{e} \tilde{F}_{e}+\frac{\tilde{\rho}_{e}}{\tilde{\rho}_{e}+\tilde{\rho}_{i} \frac{m_{e}}{m_{i}}} \frac{\partial \tilde{p}_{v}}{\partial \tilde{\xi}}\right]\right)=0 ;
\end{aligned}
$$

where

$$
\tilde{F}_{e}=\frac{\partial \tilde{\psi}}{\partial \tilde{\xi}}, \quad \tilde{F}_{i}=-\frac{m_{e}}{m_{i}} \frac{\partial \tilde{\psi}}{\partial \tilde{\xi}} .
$$

Let us derivate the dimensionless energy equation for positive ion component.

$$
\begin{aligned}
& \frac{\partial}{\partial t}\left\{\rho_{i} u^{2}+3 p_{i}-\tau_{i}\left[\frac{\partial}{\partial t}\left(\rho_{i} u^{2}+3 p_{i}\right)+\frac{\partial}{\partial x}\left(\rho_{i} u^{3}+5 p_{i} u\right)-2 \rho_{i} F_{i, e f f} u\right]\right\} \\
& +\frac{\partial}{\partial x}\left\{\rho_{i} u^{3}+5 p_{i} u-\tau_{i}\left[\frac{\partial}{\partial t}\left(\rho_{i} u^{3}+5 p_{i} u\right)\right.\right. \\
& \left.\left.+\frac{\partial}{\partial x}\left(\rho_{i} u^{4}+8 p_{i} u^{2}+5 \frac{p_{i}^{2}}{\rho_{i}}\right)-F_{i, \text { eff }}\left(3 \rho_{i} u^{2}+5 p_{i}\right)\right]\right\} \\
& -2 u \rho_{i} F_{i, \text { eff }}+2 \tau_{i} F_{i, \text { eff }}\left[\frac{\partial}{\partial t}\left(\rho_{i} u\right)+\frac{\partial}{\partial x}\left(\rho_{i} u^{2}+p_{i}\right)-\rho_{i} F_{i, \text { eff }}\right]=-\frac{p_{i}-p_{e}}{\tau_{e i}},
\end{aligned}
$$

or

$$
\begin{aligned}
& -\frac{\partial}{\partial \tilde{\xi}}\left\{\tilde{\rho}_{i} \tilde{u}^{2}+3 \tilde{p}_{i}-\tau_{i} \frac{u_{0}}{x_{0}}\left[-\frac{\partial}{\partial \tilde{\xi}}\left(\tilde{\rho}_{i} \tilde{u}^{2}+3 \tilde{p}_{i}\right)+\frac{\partial}{\partial \tilde{\xi}}\left(\tilde{\rho}_{i} \tilde{u}^{3}+5 \tilde{p}_{i} \tilde{u}\right)-2 \tilde{\rho}_{i} \tilde{F}_{i, e f f} \tilde{u}\right]\right\} \\
& +\frac{\partial}{\partial \tilde{\xi}}\left\{\tilde{\rho}_{i} \tilde{u}^{3}+5 \tilde{p}_{i} \tilde{u}-\tau_{i} \frac{u_{0}}{x_{0}}\left[-\frac{\partial}{\partial \tilde{\xi}}\left(\tilde{\rho}_{i} \tilde{u}^{3}+5 \tilde{p}_{i} \tilde{u}\right)\right.\right. \\
& \left.\left.+\frac{\partial}{\partial \tilde{\xi}}\left(\tilde{\rho}_{i} \tilde{u}^{4}+8 \tilde{p}_{i} \tilde{u}^{2}+5 \frac{\tilde{p}_{i}^{2}}{\tilde{\rho}_{i}}\right)-\tilde{F}_{i, e f f}\left(3 \tilde{\rho}_{i} \tilde{u}^{2}+5 \tilde{p}_{i}\right)\right]\right\} \\
& -2 \tilde{u} \tilde{\rho}_{i} \tilde{F}_{i, e f f}+2 \tau_{i} \tilde{F}_{i, e f f} \frac{u_{0}}{x_{0}}\left[-\frac{\partial}{\partial \tilde{\xi}}\left(\tilde{\rho}_{i} \tilde{u}\right)+\frac{\partial}{\partial \tilde{\xi}^{\prime}}\left(\tilde{\rho}_{i} \tilde{u}^{2}+\tilde{p}_{i}\right)-\tilde{\rho}_{i} \tilde{F}_{i, e f f}\right]=-t_{0} \frac{\tilde{p}_{i}-\tilde{p}_{e}}{\tau_{e i}} .
\end{aligned}
$$

Using the relations $\tau_{i}=\frac{\hbar}{m_{i} u^{2}}, \frac{\hbar}{m_{e} u_{0}}=x_{0}$ we find

$$
\begin{aligned}
& -\frac{\partial}{\partial \tilde{\xi}}\left\{\tilde{\rho}_{i} \tilde{u}^{2}+3 \tilde{p}_{i}-\frac{1}{\tilde{u}^{2}} \frac{m_{e}}{m_{i}}\left[-\frac{\partial}{\partial \tilde{\xi}}\left(\tilde{\rho}_{i} \tilde{u}^{2}+3 \tilde{p}_{i}\right)+\frac{\partial}{\partial \tilde{\xi}}\left(\tilde{\rho}_{i} \tilde{u}^{3}+5 \tilde{p}_{i} \tilde{u}\right)-2 \tilde{\rho}_{i} \tilde{F}_{i, e f f} \tilde{u}\right]\right\} \\
& +\frac{\partial}{\partial \tilde{\xi}}\left\{\tilde{\rho}_{i} \tilde{u}^{3}+5 \tilde{p}_{i} \tilde{u}-\frac{1}{\tilde{u}^{2}} \frac{m_{e}}{m_{i}}\left[-\frac{\partial}{\partial \tilde{\xi}}\left(\tilde{\rho}_{i} \tilde{u}^{3}+5 \tilde{p}_{i} \tilde{u}\right)\right.\right.
\end{aligned}
$$




$$
\begin{aligned}
& \left.\left.+\frac{\partial}{\partial \tilde{\xi}}\left(\tilde{\rho}_{i} \tilde{u}^{4}+8 \tilde{p}_{i} \tilde{u}^{2}+5 \frac{\tilde{p}_{i}^{2}}{\tilde{\rho}_{i}}\right)-\tilde{F}_{i, \text { eff }}\left(3 \tilde{\rho}_{i} \tilde{u}^{2}+5 \tilde{p}_{i}\right)\right]\right\} \\
& -2 \tilde{u} \tilde{\rho}_{i} \tilde{F}_{i, e f f}+2 \tilde{F}_{i, \text { eff }} \frac{1}{\tilde{u}^{2}} \frac{m_{e}}{m_{i}}\left[-\frac{\partial}{\partial \tilde{\xi}}\left(\tilde{\rho}_{i} \tilde{u}\right)+\frac{\partial}{\partial \tilde{\xi}}\left(\tilde{\rho}_{i} \tilde{u}^{2}+\tilde{p}_{i}\right)-\tilde{\rho}_{i} \tilde{F}_{i, e f f}\right]=-t_{0} \frac{\tilde{p}_{i}-\tilde{p}_{e}}{\tau_{e i}}
\end{aligned}
$$

After introduction the $\tau_{e i}$ nonlocal parameter $u^{2}\left(m_{e}+m_{i}\right) \tau_{e i}=\hbar$ and

$$
\begin{aligned}
\frac{\hbar}{m_{e} u_{0}}= & x_{0} \text {, we have } \\
& \frac{\partial}{\partial \tilde{\xi}}\left\{\tilde{\rho}_{i} \tilde{u}^{3}+5 \tilde{p}_{i} \tilde{u}-\tilde{\rho}_{i} \tilde{u}^{2}-3 \tilde{p}_{i}\right\}+\frac{\partial}{\partial \tilde{\xi}}\left\{\frac { 1 } { \tilde { u } ^ { 2 } } \frac { m _ { e } } { m _ { i } } \left[-\frac{\partial}{\partial \tilde{\xi}}\left(\tilde{\rho}_{i} \tilde{u}^{2}+3 \tilde{p}_{i}\right)\right.\right. \\
& \left.\left.+\frac{\partial}{\partial \tilde{\xi}}\left(\tilde{\rho}_{i} \tilde{u}^{3}+5 \tilde{p}_{i} \tilde{u}\right)-2 \tilde{\rho}_{i} \tilde{F}_{i, e f f} \tilde{u}\right]\right\}+\frac{\partial}{\partial \tilde{\xi}}\left\{\frac { 1 } { \tilde { u } ^ { 2 } } \frac { m _ { e } } { m _ { i } } \left[\frac{\partial}{\partial \tilde{\xi}}\left(\tilde{\rho}_{i} \tilde{u}^{3}+5 \tilde{p}_{i} \tilde{u}\right)\right.\right. \\
& \left.\left.-\frac{\partial}{\partial \tilde{\xi}}\left(\tilde{\rho}_{i} \tilde{u}^{4}+8 \tilde{p}_{i} \tilde{u}^{2}+5 \frac{\tilde{p}_{i}^{2}}{\tilde{\rho}_{i}}\right)+\tilde{F}_{i, e f f}\left(3 \tilde{\rho}_{i} \tilde{u}^{2}+5 \tilde{p}_{i}\right)\right]\right\} \\
& -2 \tilde{u} \tilde{\rho}_{i} \tilde{F}_{i, e f f}+2 \tilde{F}_{i, e f f} \frac{1}{\tilde{u}^{2}} \frac{m_{e}}{m_{i}}\left[-\frac{\partial}{\partial \tilde{\xi}}\left(\tilde{\rho}_{i} \tilde{u}\right)+\frac{\partial}{\partial \tilde{\xi}}\left(\tilde{\rho}_{i} \tilde{u}^{2}+\tilde{p}_{i}\right)-\tilde{\rho}_{i} \tilde{F}_{i, e f f}\right] \\
= & -\left(\tilde{p}_{i}-\tilde{p}_{e}\right) \tilde{u}^{2}\left(1+\frac{m_{i}}{m_{e}}\right),
\end{aligned}
$$

and finally

$$
\begin{aligned}
& \frac{\partial}{\partial \tilde{\xi}}\left\{\tilde{\rho}_{i} \tilde{u}^{3}+5 \tilde{p}_{i} \tilde{u}-\tilde{\rho}_{i} \tilde{u}^{2}-3 \tilde{p}_{i}\right\} \\
& +\frac{\partial}{\partial \tilde{\xi}}\left\{\frac{1}{\tilde{u}^{2}} \frac{m_{e}}{m_{i}}\left[\frac{\partial}{\partial \tilde{\xi}}\left(2 \tilde{\rho}_{i} \tilde{u}^{3}+10 \tilde{p}_{i} \tilde{u}-\tilde{\rho}_{i} \tilde{u}^{4}-\tilde{\rho}_{i} \tilde{u}^{2}-3 \tilde{p}_{i}-8 \tilde{p}_{i} \tilde{u}^{2}-5 \frac{\tilde{p}_{i}^{2}}{\tilde{\rho}_{i}}\right)\right]\right\} \\
& +\frac{\partial}{\partial \tilde{\xi}}\left\{\frac{1}{\tilde{u}^{2}} \frac{m_{e}}{m_{i}}\left(\tilde{F}_{i}+\frac{1}{\rho_{i}+\tilde{\rho}_{e} \frac{m_{i}}{m_{e}}} \frac{\partial \tilde{p}_{v}}{\partial \tilde{\xi}}\right)\left[3 \tilde{\rho}_{i} \tilde{u}^{2}+5 \tilde{p}_{i}-2 \tilde{\rho}_{i} \tilde{u}\right]\right\} \\
& -2 \tilde{u} \tilde{\rho}_{i}\left(\tilde{F}_{i}+\frac{1}{\rho_{i}+\tilde{\rho}_{e} \frac{m_{i}}{m_{e}}} \frac{\partial \tilde{p}_{v}}{\partial \tilde{\xi}}\right)+2\left(\tilde{F}_{i}+\frac{1}{\rho_{i}+\tilde{\rho}_{e} \frac{m_{i}}{m_{e}}} \frac{\partial \tilde{p}_{v}}{\partial \tilde{\xi}}\right) \frac{1}{\tilde{u}^{2}} \frac{m_{e}}{m_{i}} \\
& \left.\times \frac{\partial}{\partial \tilde{\xi}}\left(\tilde{\rho}_{i} \tilde{u}^{2}+\tilde{p}_{i}-\tilde{\rho}_{i} \tilde{u}\right)-\tilde{\rho}_{i}\left(\tilde{F}_{i}+\frac{1}{\rho_{i}+\tilde{\rho}_{e} \frac{m_{i}}{m_{e}}} \frac{\partial \tilde{p}_{v}}{\partial \tilde{\xi}}\right)\right] \\
& =-\left(\tilde{p}_{i}-\tilde{p}_{e}\right) \tilde{u}^{2}\left(1+\frac{m_{i}}{m_{e}}\right),
\end{aligned}
$$

Let's transform of the energy equation for electron component to the dimensionless form: 


$$
\begin{aligned}
& \frac{\partial}{\partial t}\left\{\rho_{e} u^{2}+3 p_{e}-\tau_{e}\left[\frac{\partial}{\partial t}\left(\rho_{e} u^{2}+3 p_{e}\right)+\frac{\partial}{\partial x}\left(\rho_{e} u^{3}+5 p_{e} u\right)-2 \rho_{e} F_{e, e f f} u\right]\right\} \\
& +\frac{\partial}{\partial x}\left\{\rho_{e} u^{3}+5 p_{e} u-\tau_{e}\left[\frac{\partial}{\partial t}\left(\rho_{e} u^{3}+5 p_{e} u\right)\right.\right. \\
& \left.\left.+\frac{\partial}{\partial x}\left(\rho_{e} u^{4}+8 p_{e} u^{2}+5 \frac{p_{e}^{2}}{\rho_{e}}\right)-F_{e, e f f}\left(3 \rho_{e} u^{2}+5 p_{e}\right)\right]\right\} \\
& -2 u \rho_{e} F_{e, e f f}+2 \tau_{e} F_{e, e f f}\left[\frac{\partial}{\partial t}\left(\rho_{e} u\right)+\frac{\partial}{\partial x}\left(\rho_{e} u^{2}+p_{e}\right)-\rho_{e} F_{e, e f f}\right]=-\frac{p_{e}-p_{i}}{\tau_{e i}},
\end{aligned}
$$

or

$$
\begin{aligned}
& -\frac{\partial}{\partial \tilde{\xi}}\left\{\tilde{\rho}_{e} \tilde{u}^{2}+3 \tilde{p}_{e}-\tau_{e} \frac{u_{0}}{x_{0}}\left[-\frac{\partial}{\partial \tilde{\xi}}\left(\tilde{\rho}_{e} \tilde{u}^{2}+3 \tilde{p}_{e}\right)+\frac{\partial}{\partial \tilde{\xi}}\left(\tilde{\rho}_{e} \tilde{u}^{3}+5 \tilde{p}_{e} \tilde{u}\right)\right.\right. \\
& \left.\left.-2 \tilde{\rho}_{e} F_{e, e f f} \tilde{u} \frac{x_{0}}{u_{0}^{2}}\right]\right\}+\frac{\partial}{\partial \tilde{\xi}}\left\{\tilde{\rho}_{e} \tilde{u}^{3}+5 \tilde{p}_{e} \tilde{u}-\tau_{e} \frac{u_{0}}{x_{0}}\left[-\frac{\partial}{\partial \tilde{\xi}}\left(\tilde{\rho}_{e} \tilde{u}^{3}+5 \tilde{p}_{e} \tilde{u}\right)\right.\right. \\
& \left.\left.+\frac{\partial}{\partial \tilde{\xi}}\left(\tilde{\rho}_{e} \tilde{u}^{4}+8 \tilde{p}_{e} \tilde{u}^{2}+5 \frac{\tilde{p}_{e}^{2}}{\tilde{\rho}_{e}}\right)-\frac{x_{0}}{u_{0}^{2}} F_{i, e f f}\left(3 \tilde{\rho}_{i} \tilde{u}^{2}+5 \tilde{p}_{i}\right)\right]\right\} \\
& -2 \tilde{u} \tilde{\rho}_{e} F_{e, e f f} \frac{x_{0}}{u_{0}^{2}}+2 \tau_{e} F_{e, e f f} \frac{1}{u_{0}}\left[-\frac{\partial}{\partial \tilde{\xi}}\left(\tilde{\rho}_{e} \tilde{u}\right)+\frac{\partial}{\partial \tilde{\xi}}\left(\tilde{\rho}_{e} \tilde{u}^{2}+\tilde{p}_{e}\right)-\tilde{\rho}_{e} F_{e, e f f} \frac{x_{0}}{u_{0}^{2}}\right] \\
& =-t_{0} \frac{\tilde{p}_{e}-\tilde{p}_{i}}{\tau_{e i}}
\end{aligned}
$$

or after using $\tau_{e}=\frac{\hbar}{m_{e} u^{2}}, \frac{\hbar}{m_{e} u_{0}}=x_{0}$ we reach the equation

$$
\begin{aligned}
& -\frac{\partial}{\partial \tilde{\xi}}\left\{\tilde{\rho}_{e} \tilde{u}^{2}+3 \tilde{p}_{e}-\frac{1}{\tilde{u}^{2}}\left[-\frac{\partial}{\partial \tilde{\xi}}\left(\tilde{\rho}_{e} \tilde{u}^{2}+3 \tilde{p}_{e}\right)+\frac{\partial}{\partial \tilde{\xi}}\left(\tilde{\rho}_{e} \tilde{u}^{3}+5 \tilde{p}_{e} \tilde{u}\right)-2 \tilde{\rho}_{e} \tilde{F}_{e, e f f} \tilde{u}\right]\right\} \\
& +\frac{\partial}{\partial \tilde{\xi}}\left\{\tilde{\rho}_{e} \tilde{u}^{3}+5 \tilde{p}_{e} \tilde{u}-\frac{1}{\tilde{u}^{2}}\left[-\frac{\partial}{\partial \tilde{\xi}}\left(\tilde{\rho}_{e} \tilde{u}^{3}+5 \tilde{p}_{e} \tilde{u}\right)\right.\right. \\
& \left.\left.+\frac{\partial}{\partial \tilde{\xi}}\left(\tilde{\rho}_{e} \tilde{u}^{4}+8 \tilde{p}_{e} \tilde{u}^{2}+5 \frac{\tilde{p}_{e}^{2}}{\tilde{\rho}_{e}}\right)-\tilde{F}_{i, e f f}\left(3 \tilde{\rho}_{i} \tilde{u}^{2}+5 \tilde{p}_{i}\right)\right]\right\} \\
& -2 \tilde{u} \tilde{\rho}_{e} \tilde{F}_{e, e f f}+2 \tilde{F}_{e, e f f} \frac{1}{\tilde{u}^{2}}\left[-\frac{\partial}{\partial \tilde{\xi}}\left(\tilde{\rho}_{e} \tilde{u}\right)+\frac{\partial}{\partial \tilde{\xi}}\left(\tilde{\rho}_{e} \tilde{u}^{2}+\tilde{p}_{e}\right)-\tilde{\rho}_{e} \tilde{F}_{e, e f f}\right] \\
& =-t_{0} \frac{\tilde{p}_{e}-\tilde{p}_{i}}{\tau_{e i}}
\end{aligned}
$$

As before we use $u^{2}\left(m_{e}+m_{i}\right) \tau_{e i}=\hbar$ and $\frac{\hbar}{m_{e} u_{0}}=x_{0}$, then

$$
\begin{aligned}
& \frac{\partial}{\partial \tilde{\xi}}\left\{\tilde{\rho}_{e} \tilde{u}^{3}+5 \tilde{p}_{e} \tilde{u}-\tilde{\rho}_{e} \tilde{u}^{2}-3 \tilde{p}_{e}\right\} \\
& +\frac{\partial}{\partial \tilde{\xi}}\left\{\frac{1}{\tilde{u}^{2}}\left[\frac{\partial}{\partial \tilde{\xi}}\left(2 \tilde{\rho}_{e} \tilde{u}^{3}+10 \tilde{p}_{e} \tilde{u}-\tilde{\rho}_{e} \tilde{u}^{4}-\tilde{\rho}_{e} \tilde{u}^{2}-3 \tilde{p}_{e}-8 \tilde{p}_{e} \tilde{u}^{2}-5 \frac{\tilde{p}_{e}^{2}}{\tilde{\rho}_{e}}\right)\right]\right\} \\
& +\frac{\partial}{\partial \tilde{\xi}}\left\{\frac{1}{\tilde{u}^{2}} \tilde{F}_{e}\left[3 \tilde{\rho}_{e} \tilde{u}^{2}+5 \tilde{p}_{e}-2 \tilde{\rho}_{e} \tilde{u}\right]\right\}-2 \tilde{u} \tilde{\rho}_{e} \tilde{F}_{e, e f f}
\end{aligned}
$$




$$
+2 \tilde{F}_{e, e f f} \frac{1}{\tilde{u}^{2}}\left[\frac{\partial}{\partial \tilde{\xi}}\left(\tilde{\rho}_{e} \tilde{u}^{2}+\tilde{p}_{e}-\tilde{\rho}_{e} \tilde{u}\right)-\tilde{\rho}_{e} \tilde{F}_{e, e f f}\right]=-\left(\tilde{p}_{e}-\tilde{p}_{i}\right) \tilde{u}^{2}\left(1+\frac{m_{i}}{m_{e}}\right) .
$$

Writing in the explicit form we use for example

$$
\tilde{F}_{e, e f f}=\tilde{F}_{e}+\frac{1}{\tilde{\rho}_{e}+\tilde{\rho}_{i} \frac{m_{e}}{m_{i}}} \frac{\partial \tilde{p}_{v}}{\partial \tilde{\xi}},
$$

then

$$
\begin{aligned}
& \frac{\partial}{\partial \tilde{\xi}}\left\{\tilde{\rho}_{e} \tilde{u}^{3}+5 \tilde{p}_{e} \tilde{u}-\tilde{\rho}_{e} \tilde{u}^{2}-3 \tilde{p}_{e}\right\} \\
& +\frac{\partial}{\partial \tilde{\xi}}\left\{\frac{1}{\tilde{u}^{2}}\left[\frac{\partial}{\partial \tilde{\xi}}\left(2 \tilde{\rho}_{e} \tilde{u}^{3}+10 \tilde{p}_{e} \tilde{u}-\tilde{\rho}_{e} \tilde{u}^{4}-\tilde{\rho}_{e} \tilde{u}^{2}-3 \tilde{p}_{e}-8 \tilde{p}_{e} \tilde{u}^{2}-5 \frac{\tilde{p}_{e}^{2}}{\tilde{\rho}_{e}}\right)\right]\right\} \\
& +\frac{\partial}{\partial \tilde{\xi}}\left\{\frac{1}{\tilde{u}^{2}}\left(\tilde{F}_{e}+\frac{1}{\tilde{\rho}_{e}+\tilde{\rho}_{i}} \frac{m_{e}}{m_{i}} \frac{\partial \tilde{p}_{v}}{\partial \tilde{\xi}}\right)\left[3 \tilde{\rho}_{e} \tilde{u}^{2}+5 \tilde{p}_{e}-2 \tilde{\rho}_{e} \tilde{u}\right]\right\} \\
& -2 \tilde{u} \tilde{\rho}_{e}\left(\tilde{F}_{e}+\frac{1}{\left.\tilde{\rho}_{e}+\tilde{\rho}_{i} \frac{m_{e}}{m_{i}} \frac{\partial \tilde{p}_{v}}{\partial \tilde{\xi}}\right)+2\left(\tilde{F}_{e}+\frac{1}{\tilde{\rho}_{e}+\tilde{\rho}_{i} \frac{m_{e}}{m_{i}}} \frac{\partial \tilde{p}_{v}}{\partial \tilde{\xi}}\right) \frac{1}{\tilde{u}^{2}}}\right. \\
& \times\left[\frac{\partial}{\partial \tilde{\xi}}\left(\tilde{\rho}_{e} \tilde{u}^{2}+\tilde{p}_{e}-\tilde{\rho}_{e} \tilde{u}\right)-\tilde{\rho}_{e}\left(\tilde{F}_{e}+\frac{1}{\left.\left.\tilde{\rho}_{e}+\tilde{\rho}_{i} \frac{m_{e}}{m_{i}} \frac{\partial \tilde{p}_{v}}{\partial \tilde{\xi}}\right)\right]}\right.\right. \\
& =-\left(\tilde{p}_{e}-\tilde{p}_{i}\right) \tilde{u}^{2}\left(1+\frac{m_{i}}{m_{e}}\right) .
\end{aligned}
$$

If $\frac{\partial p_{v}}{\partial x}-R_{v, x}=0$, then motion equation for the PV evolution is written as follows

$$
\frac{\partial p_{v}}{\partial t}+v_{v, x} \frac{\partial p_{v}}{\partial x}+4 p_{v} \frac{\partial v_{v, x}}{\partial x}=0
$$

or to the equation which has the wave solutions

$$
\frac{\partial \tilde{p}_{v}}{\tilde{\partial} \tilde{\xi}}-\tilde{v}_{v, x} \frac{\partial \tilde{p}_{v}}{\partial \tilde{\xi}}-4 \tilde{p}_{v} \frac{\partial \tilde{v}_{v, x}}{\partial \tilde{\xi}}=0
$$

Let us demonstrate derivation of the wave PV energy equation for the case $\tau_{v} \neq 0$. From Equation (5.7) follows in the PV limit $(\rho \rightarrow 0)$

$$
\begin{aligned}
& \frac{\partial}{\partial t}\left\{3 p_{v}-\tau_{v}\left[3 \frac{\partial p_{v}}{\partial t}+5 \frac{\partial}{\partial x}\left(v_{v} p_{v}\right)-2 R_{r} v_{v}\right]\right\} \\
& +\frac{\partial}{\partial x}\left\{5 p v_{v}-\tau_{v}\left[5 \frac{\partial}{\partial t}\left(p v_{v}\right)+7 \frac{\partial}{\partial x}\left(p v_{v}^{2}\right)-2 R_{r} v_{v}^{2}\right]\right\} \\
& -\frac{\partial}{\partial x}\left(\tau_{v} \frac{\partial}{\partial x}\left(p_{v} v_{v}^{2}\right)\right)-5 \frac{\partial}{\partial x}\left(\tau_{s} \frac{\partial}{\partial x} \frac{p^{2}}{\rho}\right)=0,
\end{aligned}
$$


or

$$
\begin{aligned}
& \frac{\partial}{\partial t}\left\{3 p_{v}-\tau_{v}\left[3 \frac{\partial p_{v}}{\partial t}+5 p_{v} \frac{\partial v_{v}}{\partial x}+3 v_{v} \frac{\partial p_{v}}{\partial x}\right]\right\} \\
& +5 p_{v} \frac{\partial v_{v}}{\partial x}+3 v_{v} \frac{\partial p_{v}}{\partial x}-\frac{\partial}{\partial x}\left\{\tau_{v}\left[5 \frac{\partial}{\partial t}\left(p_{v} v_{v}\right)+5 v_{v}^{2} \frac{\partial p_{v}}{\partial x}+7 p \frac{\partial v_{v}^{2}}{\partial x}\right]\right\} \\
& -\frac{\partial}{\partial x}\left(\tau_{v} \frac{\partial}{\partial x}\left(p_{v} v_{v}^{2}\right)\right)-5 \frac{\partial}{\partial x}\left(\tau_{s} \frac{\partial}{\partial x} \frac{p^{2}}{\rho}\right)=0,
\end{aligned}
$$

Write down the energy PV Equation (9.54) for the case $\tau_{v}=$ const in the dimensionless form, we find

$$
\begin{aligned}
& 3 \frac{\partial \tilde{p}_{v}}{\partial \tilde{\xi}}-5 \tilde{p}_{v} \frac{\partial \tilde{v}_{v}}{\partial \tilde{\xi}}-3 \tilde{v}_{v} \frac{\partial \tilde{p}_{v}}{\partial \tilde{\xi}}+5 \frac{m_{e}}{m_{e}+m_{i}} \frac{\partial}{\partial \tilde{\xi}}\left(\frac{1}{\tilde{u}_{s}^{2}} \frac{\partial}{\partial \tilde{\xi}} \frac{\left(\tilde{p}_{i}+\tilde{p}_{e}\right)^{2}}{\tilde{\rho}_{i}+\tilde{\rho}_{e}}\right) \\
& +\tilde{\tau}_{v} \frac{\partial}{\partial \tilde{\xi}}\left[3 \frac{\partial \tilde{p}_{v}}{\partial \tilde{\xi}}-8 \tilde{v}_{v} \frac{\partial \tilde{p}_{v}}{\partial \tilde{\xi}}-10 \tilde{p}_{v} \frac{\partial \tilde{v}_{v}}{\partial \tilde{\xi}}+6 \tilde{v}_{v}^{2} \frac{\partial \tilde{p}_{v}}{\partial \tilde{\xi}}+16 \tilde{p}_{v} \tilde{v}_{v} \frac{\partial \tilde{v}_{v}}{\partial \tilde{\xi}}\right]=0 .
\end{aligned}
$$

The following figures reflect some results of calculations realized according to the system of of eight ordinary non-linear Equations (9.20), (9.29), (9.33), (9.38), (9.44),(9.50), (9.52) and (9.55) with the help of Maple.

Some comments to the system of Equations (9.20), (9.29), (9.33), (9.38), (9.44), (9.50), (9.52) and (9.55):

1) The problem belongs to the class of Cauchy problems.

2) In comparison with the Schrödinger theory connected with behavior of the wave function, no special conditions are applied for dependent variables including the domain of the solution existing. This domain is defined automatically in the process of the numerical solution of the concrete variant of calculations.

3) From the introduced scales

$$
u_{0}, \quad x_{0}=\frac{\hbar}{m_{e}} \frac{1}{u_{0}}, \quad \psi_{0}=\frac{m_{e}}{e} u_{0}^{2}, \quad \rho_{0}=\frac{m_{e}^{4}}{4 \pi \hbar^{2} e^{2}} u_{0}^{4}, \quad p_{0}=\rho_{0} u_{0}^{2}=\frac{m_{e}^{4}}{4 \pi \hbar^{2} e^{2}} u_{0}^{6}
$$

only two parameters are independent - the phase velocity $u_{0}$ of the quantum object, and external parameter $H$, which is proportional to Plank constant $\hbar$ and in general case should be inserted in the scale relation as $x_{0}=\frac{H}{m_{e} u_{0}}=\frac{n \hbar}{m_{e} u_{0}}$. It leads to exchange in all scales $\hbar \leftrightarrow H$.

The following notations on figures are used: r-density $\tilde{\rho}_{i}$, s-density $\tilde{\rho}_{e}$, u-velocity $\tilde{u}$, h-PV velocity, p-pressure $\tilde{p}_{i}$, q-pressure $\tilde{p}_{e}, \mathrm{w}-\mathrm{PV}$ pressure $\tilde{p}_{v}$ and v-self consistent potential $\tilde{\psi}$. Other notations: $m_{e} \rightarrow \mathrm{L}, m_{i} \rightarrow \mathrm{T}$, $\frac{\partial}{\partial \tilde{\xi}} \rightarrow \mathrm{D}$, independent variable $t$ responds to $\tilde{\xi}$. Explanations placed under all following figures, Maple program contains Maple's notations for example the expression $D(u)(0)=0$ means in the usual notations $\frac{\partial \tilde{u}}{\partial \tilde{\xi}}(0)=0$.

Let's compare the two configurations-taking into account the influence of the physical vacuum and disregarding this influence. Cauchy conditions placed 
under all following figures.

Figure 10 reflects results without the PV influence.

Figure 11 reflects results with taking into account the PV influence (but the PV nonlocal parameter $\tilde{\tau}_{v}=0$ ).

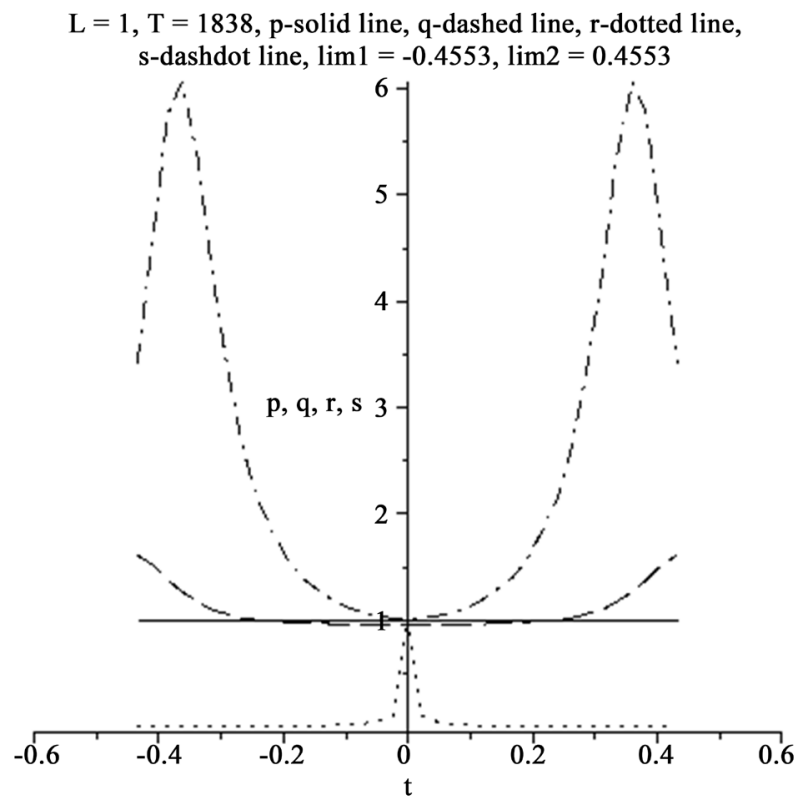

Figure 10. p-pressure $\tilde{p}_{i}$ (solid line), q-pressure $\tilde{p}_{e}$ (dashed line), r-density $\tilde{\rho}_{i}$ (dotted line), s-density $\tilde{\rho}_{e}$ (dashdot line), Cauchy conditions: A) v(0)=1, r(0)=1, s(0) $=1, \mathrm{u}(0)=1, \mathrm{p}(0)=1, \mathrm{q}(0)=0.95, \mathrm{D}(\mathrm{v})(0)=0, \mathrm{D}(\mathrm{r})(0)=0, \mathrm{D}(\mathrm{s})(0)=0, \mathrm{D}(\mathrm{u})(0)=0$, $\mathrm{D}(\mathrm{p})(0)=0, \mathrm{D}(\mathrm{q})(0)=0$

\section{$\mathrm{L}=1, \mathrm{~T}=1838, \mathrm{p}$-solid line, $\mathrm{q}$-dashed line, $\mathrm{r}$-dotted line,} s-dashdot line, $\lim 1=0.003799, \lim 2=0.004505$

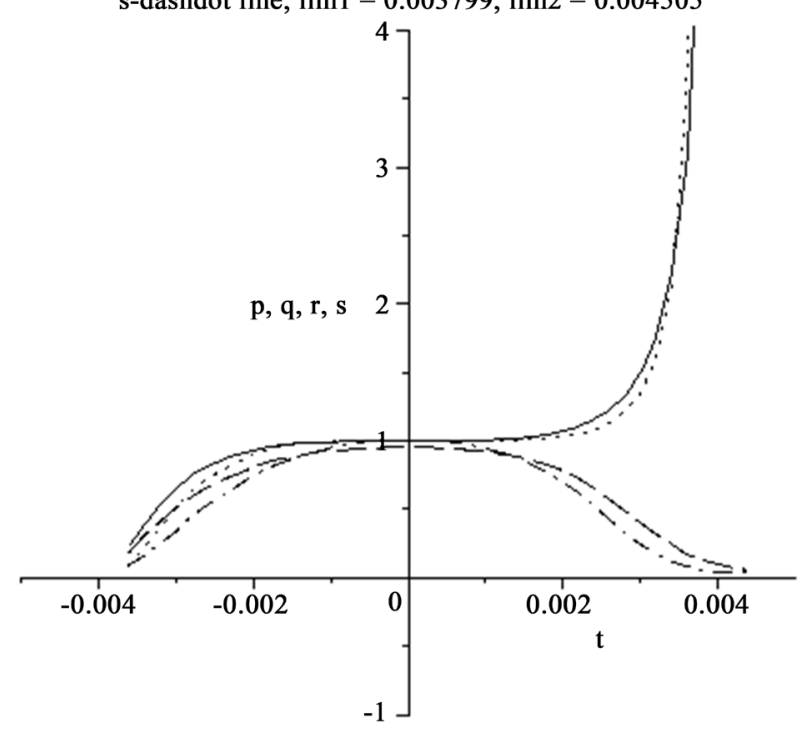

Figure 11. p-pressure $\tilde{p}_{i}$ (solid line), q-pressure $\tilde{p}_{e}$ (dashed line), r-density $\tilde{\rho}_{i}$ (dotted line), s-density $\tilde{\rho}_{e}$ (dashdot line), Cauchy conditions: $\mathrm{v}(0)=1, \mathrm{r}(0)=1, \mathrm{~s}(0)=1$, $\mathrm{u}(0)=1, \mathrm{p}(0)=1, \mathrm{q}(0)=0.95, \mathrm{D}(\mathrm{v})(0)=0, \mathrm{D}(\mathrm{r})(0)=0, \mathrm{D}(\mathrm{s})(0)=0, \mathrm{D}(\mathrm{u})(0)=0, \mathrm{D}(\mathrm{p})(0)$ $=0, \mathrm{D}(\mathrm{q})(0)=0, \mathrm{w}(0)=1, \mathrm{D}(\mathrm{w})(0)=0, \mathrm{~h}(0)=0, \mathrm{D}(\mathrm{h})(0)=1$. 
For the Cauchy conditions A) the PV influence leads to the destruction of the atom structure (like hydrogen) and appearance the object with the positive charged shell and the character size, which is smaller in hundreds times than previous object (like neutron).

Figure 12 reflects results without the PV influence, but for other Cauchy conditions type $\mathrm{B}: \mathrm{B}) \mathrm{v}(0)=1, \mathrm{r}(0)=1, \mathrm{~s}(0)=1, \mathrm{u}(0)=1, \mathrm{p}(0)=0.95, \mathrm{q}(0)=1$, $\mathrm{D}(\mathrm{v})(0)=0, \mathrm{D}(\mathrm{r})(0)=0, \mathrm{D}(\mathrm{s})(0)=0, \mathrm{D}(\mathrm{u})(0)=0, \mathrm{D}(\mathrm{p})(0)=0, \mathrm{D}(\mathrm{q})(0)=0$.

For the Cauchy conditions $\mathrm{B}$ ) the PV influence leads to the destruction of the structure with the positive shell (like neutron) and to appearance of the object with the negative charged shell and the character size, which is smaller in hundreds times than classical hydrogen atom.

Figure 13 reflects the results with the PV influence $\left(\tilde{\tau}_{v}=0\right.$ or $\left.\mathrm{D}=0\right)$.

In other words the anti-hydrogen has the very small cross section (in comparison with the hydrogen atoms), leaves the birth area and is now on the periphery of the Universe (see also [4] [6]).

But it is not the full scenario of events. We prolong our investigation. Let be now $\tilde{\tau}_{v} \neq 0$. In this case we should use the PV energy equation in the form:

$$
\begin{aligned}
& \frac{\partial}{\partial t}\left\{3 p_{v}-\tau_{v}\left[3 \frac{\partial p_{v}}{\partial t}+5 p_{v} \frac{\partial v_{v}}{\partial x}+3 v_{v} \frac{\partial p_{v}}{\partial x}\right]\right\} \\
& +5 p_{v} \frac{\partial v_{v}}{\partial x}+3 v_{v} \frac{\partial p_{v}}{\partial x}-\frac{\partial}{\partial x}\left\{\tau_{v}\left[5 \frac{\partial}{\partial t}\left(p_{v} v_{v}\right)+5 v_{v}^{2} \frac{\partial p_{v}}{\partial x}+7 p \frac{\partial v_{v}^{2}}{\partial x}\right]\right\} \\
& -\frac{\partial}{\partial x}\left(\tau_{v} \frac{\partial}{\partial x}\left(p_{v} v_{v}^{2}\right)\right)-5 \frac{\partial}{\partial x}\left(\tau_{s} \frac{\partial}{\partial x} \frac{p^{2}}{\rho}\right)=0,
\end{aligned}
$$

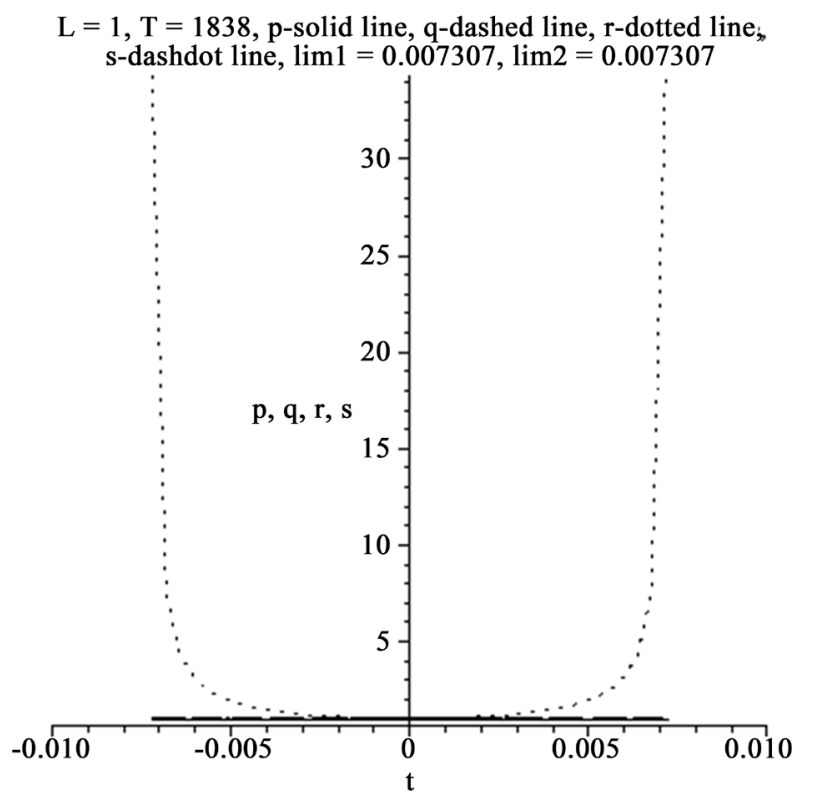

Figure 12. p-pressure $\tilde{p}_{i}$ (solid line), q-pressure $\tilde{p}_{e}$ (dashed line), r-density $\tilde{\rho}_{i}$ (dotted line), s-density $\tilde{\rho}_{e}$ (dashdot line), Cauchy conditions: B) $\mathrm{v}(0)=1, \mathrm{r}(0)=1, \mathrm{~s}(0)$ $=1, \mathrm{u}(0)=1, \mathrm{p}(0)=0.95, \mathrm{q}(0)=1, \mathrm{D}(\mathrm{v})(0)=0, \mathrm{D}(\mathrm{r})(0)=0, \mathrm{D}(\mathrm{s})(0)=0, \mathrm{D}(\mathrm{u})(0)=0$, $\mathrm{D}(\mathrm{p})(0)=0, \mathrm{D}(\mathrm{q})(0)=0$. 


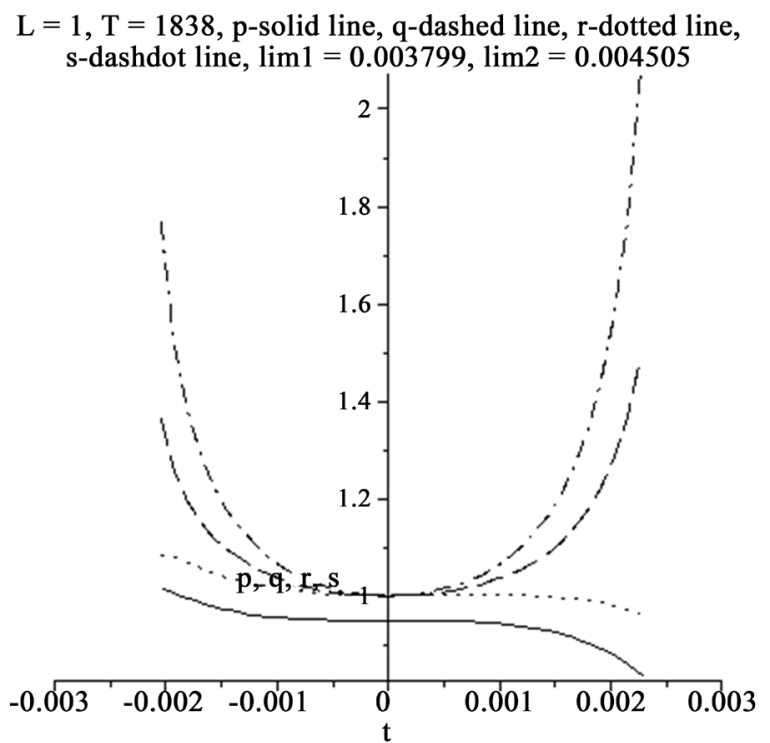

Figure 13. p-pressure $\tilde{p}_{i}$ (solid line), q-pressure $\tilde{p}_{e}$ (dashed line), r-density $\tilde{\rho}_{i}$ (dotted line), s-density $\tilde{\rho}_{e}$ (dashdot line), Cauchy conditions: $\mathrm{v}(0)=1, \mathrm{r}(0)=1, \mathrm{~s}(0)=$ $1, \mathrm{u}(0)=1, \mathrm{p}(0)=0.95, \mathrm{q}(0)=1, \mathrm{D}(\mathrm{v})(0)=0, \mathrm{D}(\mathrm{r})(0)=0, \mathrm{D}(\mathrm{s})(0)=0, \mathrm{D}(\mathrm{u})(0)=0$, $\mathrm{D}(\mathrm{p})(0)=0, \mathrm{D}(\mathrm{q})(0)=0, \mathrm{w}(0)=1, \mathrm{D}(\mathrm{w})(0)=0, \mathrm{~h}(0)=0, \mathrm{D}(\mathrm{h})(0)=1$.

As we see from Equation (9.56) the nonlocal PV parameter is in reality a function of coordinates and time. Interesting to notice, that PV loses the interaction with Matter if $\tilde{\tau} \rightarrow \infty$. If $0<\tilde{\tau}_{v}<\infty$ we should use the additional dimensionless PV energy equation in the form (9.55) supposing $\tilde{\tau}_{v}=$ const .

Let be $\tilde{\tau}_{v}=1 \quad(\mathrm{D}=1)$, we find

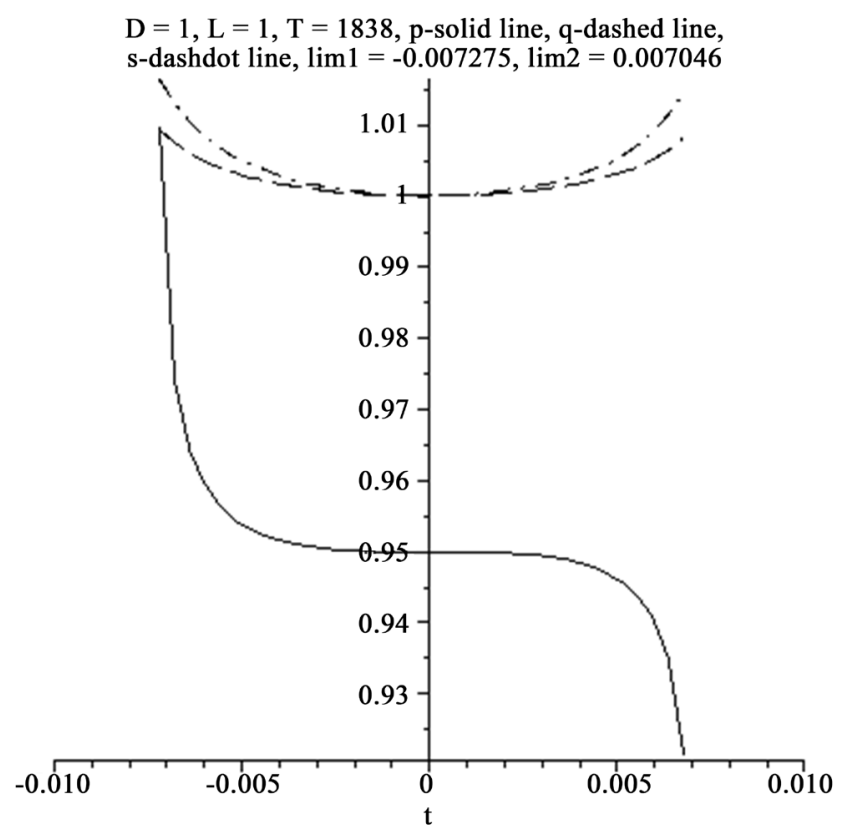

Figure 14. p-pressure $\tilde{p}_{i}$ (solid line), q-pressure $\tilde{p}_{e}$ (dashed line), s-density $\tilde{\rho}_{e}$ (dashdot line), Cauchy conditions: $\mathrm{v}(0)=1, \mathrm{r}(0)=1, \mathrm{~s}(0)=1, \mathrm{u}(0)=1, \mathrm{p}(0)=0.95, \mathrm{q}(0)=1$, $\mathrm{D}(\mathrm{v})(0)=0, \mathrm{D}(\mathrm{r})(0)=0, \mathrm{D}(\mathrm{s})(0)=0, \mathrm{D}(\mathrm{u})(0)=0, \mathrm{D}(\mathrm{p})(0)=0, \mathrm{D}(\mathrm{q})(0)=0$. 
Let us compare now the results of two calculations realized in the same conditions but for the different nonlocal PV parameters $(D=0$ and $D=1)$.

For Figure 15 and Figure 16: p-pressure $\tilde{p}_{i}$ (solid line), q-pressure $\tilde{p}_{e}$

$\mathrm{D}=0, \mathrm{~L}=1, \mathrm{~T}=1838$, $\mathrm{p}$-solid line, $\mathrm{q}$-dashed line, $\mathrm{r}$-dotted line, s-dashdot line, $\lim 1=-0.002094, \lim 2=0.002372$

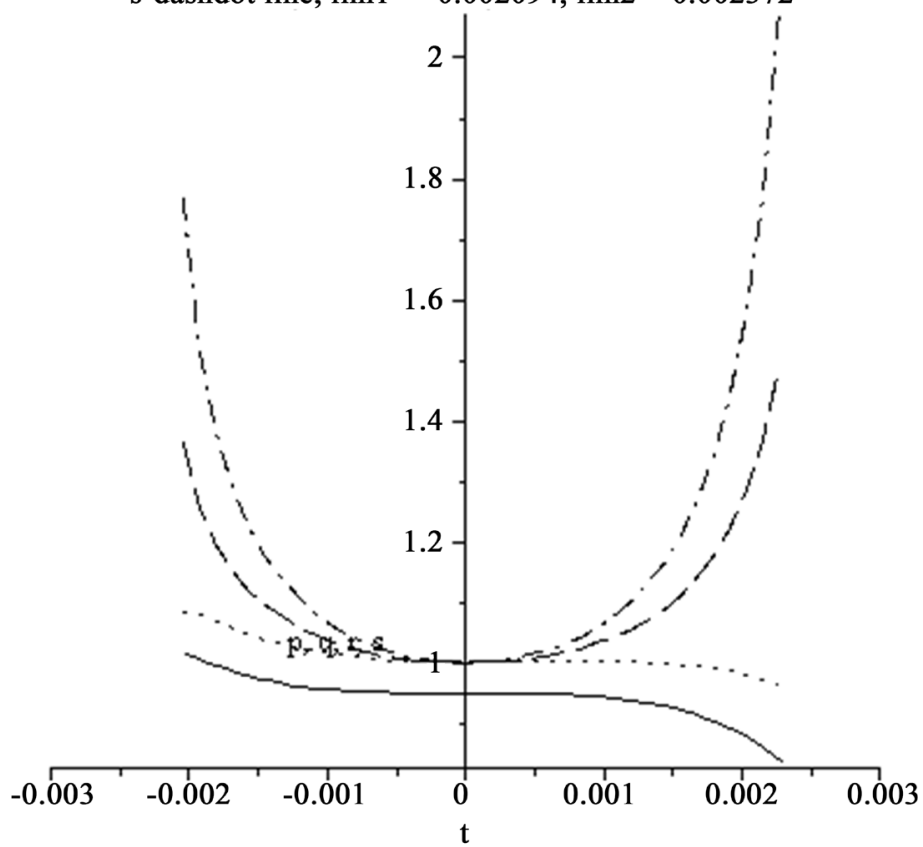

Figure 15. $p$-pressure $\tilde{p}_{i}$ (solid line), $q$-pressure $\tilde{p}_{e}$ (dashed line), r-density (dotted line), s-density $\tilde{p}_{e}$ (dashdot line). Cauchy conditions: $\mathrm{v}(0)=1, \mathrm{r}(0)=1, \mathrm{~s}(0)=1, \mathrm{u}(0)=$ $1, \mathrm{p}(0)=0.95, \mathrm{q}(0)=1, \mathrm{D}(\mathrm{v})(0)=0, \mathrm{D}(\mathrm{r})(0)=0, \mathrm{D}(\mathrm{s})(0)=0, \mathrm{D}(\mathrm{u})(0)=0, \mathrm{D}(\mathrm{p})(0)=0$, $\mathrm{D}(\mathrm{q})(0)=0, \mathrm{w}(0)=1, \mathrm{D}(\mathrm{w})(0)=0, \mathrm{~h}(0)=0, \mathrm{D}(\mathrm{h})(0)=1$.

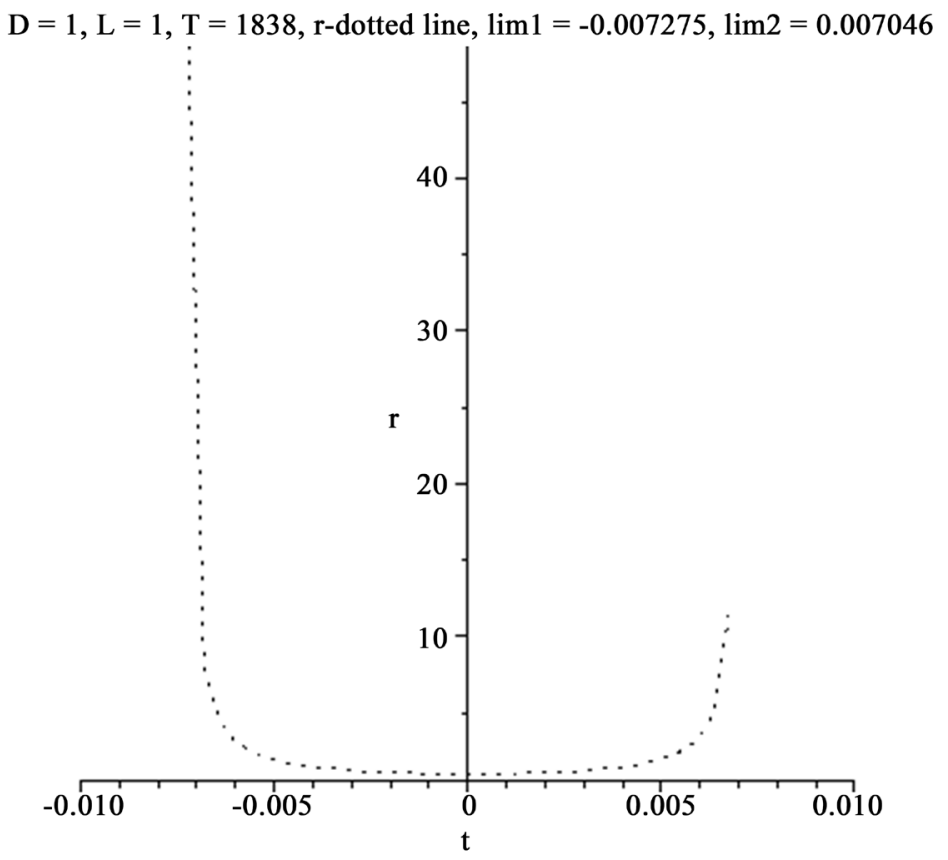

Figure 16. r-density $\tilde{p}_{i}$ (dotted line) 
(dashed line), r-density $\tilde{\rho}_{i}$ (dotted line), s-density $\tilde{\rho}_{e}$ (dashdot line), Cauchy conditions: $\mathrm{v}(0)=1, \mathrm{r}(0)=1, \mathrm{~s}(0)=1, \mathrm{u}(0)=1, \mathrm{p}(0)=0.95, \mathrm{q}(0)=1, \mathrm{D}(\mathrm{v})(0)=$ $0, \mathrm{D}(\mathrm{r})(0)=0, \mathrm{D}(\mathrm{s})(0)=0, \mathrm{D}(\mathrm{u})(0)=0, \mathrm{D}(\mathrm{p})(0)=0, \mathrm{D}(\mathrm{q})(0)=0, \mathrm{w}(0)=1$, $\mathrm{D}(\mathrm{w})(0)=0, \mathrm{~h}(0)=0, \mathrm{D}(\mathrm{h})(0)=1$.

Take a look at Figure 15 (with $\tilde{\tau}_{v}=0$, or $\mathrm{D}=0$, left here) and Figure 16 (with $\tilde{\tau}_{v}=1$ or $\mathrm{D}=1$ ), right here). These configurations have the extremely important feature (for the chosen Cauchy conditions, compare with Figure 10). In the first case $(\mathrm{D}=0)$ this object has the negative charged shell (like hydrogen), but in the second case $(D=1)$ we reveal the object with the positive charged shell with the larger cross section.

This situation can be explained be the following way. At an early stage in the development of the universe the value of the PV parameter is closer to zero. As a result the antimatter (as I wrote before) having the small cross section, leaves the central part of domain. As the universe ages, the non-locality parameter $\tilde{\tau}_{v}$ increases, which leads to the situation responding configuration 16, right.

Figure 17 and Figure 18 reflect some other calculations.

Figures 11-18 display quantum objects placed in bounded region of 1D space, all parts of this objects are moving with practically the same velocity. Important to underline that no special boundary conditions were used for all cases. Then this soliton is product of the self-organization of ionized matter.

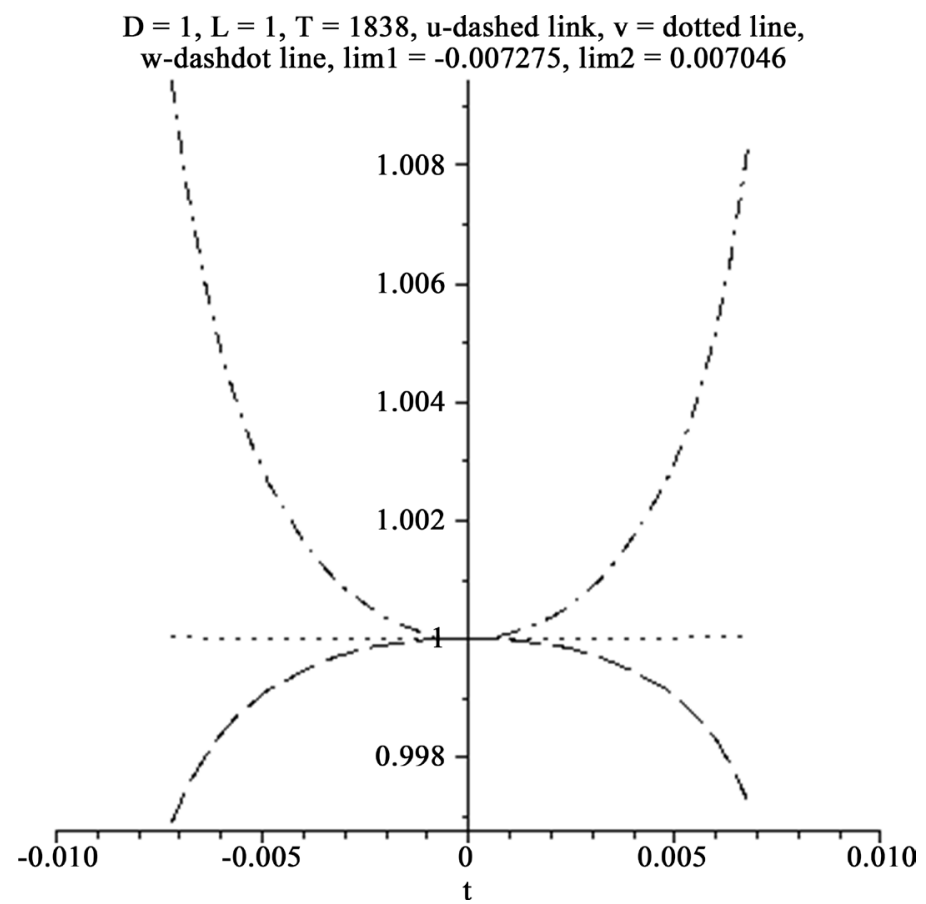

Figure 17. u-velocity $\tilde{v}$ (dashed line), v-electric potential $\tilde{\psi}$ (dotted line), w-PV pressure $\tilde{p}_{v}$ (dashdot line). $\mathrm{v}(0)=1, \mathrm{r}(0)=1, \mathrm{~s}(0)=1, \mathrm{u}(0)=1, \mathrm{p}(0)=0.95, \mathrm{q}(0)=1$, $\mathrm{D}(\mathrm{v})(0)=0, \mathrm{D}(\mathrm{r})(0)=0, \mathrm{D}(\mathrm{s})(0)=0, \mathrm{D}(\mathrm{u})(0)=0, \mathrm{D}(\mathrm{p})(0)=0, \mathrm{D}(\mathrm{q})(0)=0, \mathrm{w}(0)=1$, $\mathrm{D}(\mathrm{w})(0)=0, \mathrm{~h}(0)=0, \mathrm{D}(\mathrm{h})(0)=1$. 


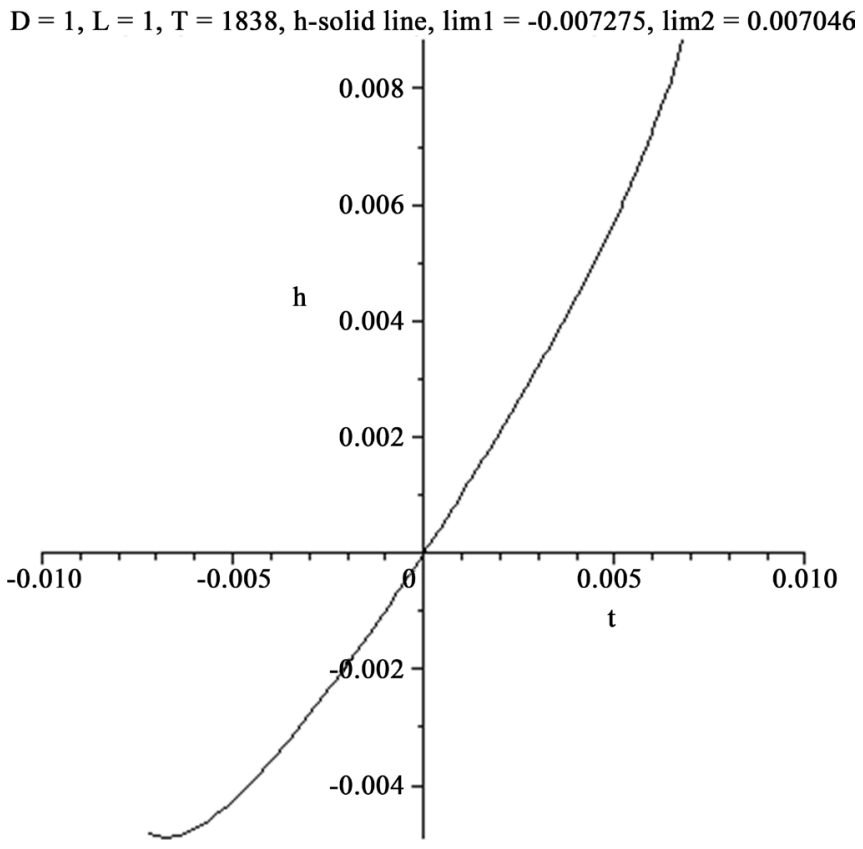

Figure 18. h-velocity $\tilde{v}_{v} \quad$ (solid line), $\mathrm{v}(0)=1, \mathrm{r}(0)=1, \mathrm{~s}(0)=1, \mathrm{u}(0)=1, \mathrm{p}(0)=0.95$, $\mathrm{q}(0)=1, \mathrm{D}(\mathrm{v})(0)=0, \mathrm{D}(\mathrm{r})(0)=0, \mathrm{D}(\mathrm{s})(0)=0, \mathrm{D}(\mathrm{u})(0)=0, \mathrm{D}(\mathrm{p})(0)=0, \mathrm{D}(\mathrm{q})(0)=0, \mathrm{w}(0)$ $=1, \mathrm{D}(\mathrm{w})(0)=0, \mathrm{~h}(0)=0, \mathrm{D}(\mathrm{h})(0)=1$.

\section{About the Energy Echange between Ordinary Matter and Physical Vacuum}

Let us consider the spherical 1D stationary flow and its interaction with the surrounding physical vacuum. It means that we should introduce into consideration two kinds of nonlocal paramaters for matter $\tau_{s}$ and for physical vacuum (PV) $\tau_{v}$. The generalized nonlocal hydrodynamic equations can be written as follows (see also the system of Equations (5.1) - (5.7)):

continuity equation

$$
\frac{1}{r^{2}} \frac{\partial}{\partial r}\left\{r^{2}\left\{\rho v_{0 r}-\tau_{s}\left[\frac{1}{r^{2}} \frac{\partial\left(r^{2} \rho v_{0 r}^{2}\right)}{\partial r}-R_{r}\right]\right\}\right\}-\frac{1}{r^{2}} \frac{\partial}{\partial r}\left(\tau_{s} r^{2} \frac{\partial p}{\partial r}\right)=0 .
$$

This equation can be immediately integrated

$$
\rho v_{0 r}-\tau_{s}\left[\frac{1}{r^{2}} \frac{\partial\left(r^{2} \rho v_{0 r}^{2}\right)}{\partial r}+\frac{\partial p}{\partial r}-R_{r}\right]=0,
$$

where $R_{r}$ is the external force acting in the radial direction on the volume unit.

Motion equation for matter for the stationary case is written as

$$
\begin{aligned}
& \frac{\partial p}{\partial r}-R_{r}+\tau_{s} F_{r} \frac{1}{r^{2}} \frac{\partial\left(r^{2} \rho v_{0 r}\right)}{\partial r}+\frac{1}{r^{2}} \frac{\partial}{\partial r}\left\{r^{2}\left\{\rho v_{0 r}^{2}-\tau_{s}\left[\frac{1}{r^{2}} \frac{\partial\left(r^{2} \rho v_{0 r}^{3}\right)}{\partial r}-2 R_{r} v_{0 r}\right]\right\}\right\} \\
& -2 \frac{\partial}{\partial r}\left(\frac{\tau_{s}}{r^{2}} \frac{\partial\left(r^{2} p v_{0 r}\right)}{\partial r}\right)-\frac{1}{r^{2}} \frac{\partial}{\partial r}\left(\tau_{s} r^{2} \frac{\partial\left(p v_{0 r}\right)}{\partial r}\right)=0,
\end{aligned}
$$


where $F_{r}$ is the external force acting in the radial direction on the mass unit,

$$
R_{r}=\rho F_{r} .
$$

Equation (10.3) can be written in the form $\left(\tau_{s}=\right.$ const $)$

$$
\begin{aligned}
& r^{2}\left(\frac{\partial p}{\partial r}-R_{r}\right)+\tau_{s} F_{r} \frac{\partial\left(r^{2} \rho v_{0 r}\right)}{\partial r}+\frac{\partial}{\partial r}\left(r^{2} \rho v_{0 r}^{2}\right)-\tau_{s} \frac{\partial^{2}\left(r^{2} \rho v_{0 r}^{3}\right)}{\partial r^{2}} \\
& +2 \tau_{s} \frac{\partial}{\partial r}\left(r^{2} R_{r} v_{0 r}\right)-2 \tau_{s} r^{2} \frac{\partial}{\partial r}\left(\frac{1}{r^{2}} \frac{\partial\left(r^{2} p v_{0 r}\right)}{\partial r}\right)-\tau_{s} \frac{\partial}{\partial r}\left(r^{2} \frac{\partial\left(p v_{0 r}\right)}{\partial r}\right)=0 .
\end{aligned}
$$

Energy equation for the matter for the stationary case has the form

$$
\begin{aligned}
& \frac{\partial}{\partial r}\left\{r^{2} v_{0 r}\left(\rho v_{0 r}^{2}+5 p\right)\right\}-\tau_{s} \frac{\partial^{2}}{\partial r^{2}}\left(r^{2}\left(\rho v_{0 r}^{2}+7 p\right) v_{0 r}^{2}\right) \\
& +\tau_{s} \frac{\partial}{\partial r}\left(3 r^{2} R_{r} v_{0 r}^{2}+5 r^{2} p F\right)-2 R_{r} v_{0 r} r^{2}+2 \tau_{s} F_{r} \frac{\partial}{\partial r}\left(r^{2} \rho v_{0 r}^{2}\right) \\
& +2 \tau_{s} F_{r} r^{2}\left(\frac{\partial p}{\partial r}-R_{r}\right)-\tau_{s} \frac{\partial}{\partial r}\left(r^{2} \frac{\partial}{\partial r}\left(p v_{0 r}^{2}\right)\right)-5 \tau_{s} \frac{\partial}{\partial r}\left(r^{2} \frac{\partial}{\partial r}\left(\frac{p^{2}}{\rho}\right)\right)=0,
\end{aligned}
$$

We should add to the previous Equations (10.2), (10.3) and (10.6) the selfconsistent equations describing the PV motion. The nonlocal parameter $\tau_{v}$ for PV should be introduced. Then for the matter and PV description we find

$$
\begin{gathered}
\rho_{s} v_{s}-\tau_{s}\left[\frac{1}{r^{2}} \frac{\partial\left(r^{2} \rho_{s} v_{s}^{2}\right)}{\partial r}+\frac{\partial p_{s}}{\partial r}-\frac{\partial p_{v}}{\partial r}\right]=0, \\
r^{2} \frac{\partial}{\partial r}\left(p_{s}-p_{v}\right)+\tau_{s} \frac{\partial p_{v}}{\partial r} \frac{1}{\rho_{s}} \frac{\partial\left(r^{2} \rho_{s} v_{s}\right)}{\partial r}+\frac{\partial}{\partial r}\left\{r^{2} \rho_{s} v_{s}^{2}\right\} \\
-\tau_{s} \frac{\partial}{\partial r}\left[\frac{\partial\left(r^{2} \rho_{s} v_{s}^{3}\right)}{\partial r}\right]+2 \tau_{s} \frac{\partial}{\partial r}\left\{r^{2} \frac{\partial p_{v}}{\partial r} v_{s}\right\} \\
-2 \tau_{s} r^{2} \frac{\partial}{\partial r}\left(\frac{1}{r^{2}} \frac{\partial\left(r^{2} p_{s} v_{s}\right)}{\partial r}\right)-\tau_{s} \frac{\partial}{\partial r}\left(r^{2} \frac{\partial\left(p_{s} v_{s}\right)}{\partial r}\right)=0 . \\
\frac{\partial}{\partial r}\left\{r^{2} v_{s}\left(\rho v_{0 r}^{2}+5 p_{s}\right)\right\}-\tau_{s} \frac{\partial}{\partial r}\left\{\frac{\partial}{\partial r}\left(r^{2}\left(\rho_{s} v_{s}^{2}+7 p_{s}\right) v_{s}^{2}\right)\right\} \\
+\tau_{s} \frac{\partial}{\partial r}\left\{3 r^{2} \frac{\partial p_{v}}{\partial r} v_{s}^{2}+5 r^{2} \frac{p_{s}}{\rho_{s}} \frac{\partial p_{v}}{\partial r}\right\}-2 r^{2} \frac{\partial p_{v}}{\partial r} v_{s} \\
+2 \tau_{s} \frac{1}{\rho_{s}} \frac{\partial p_{v}}{\partial r} \frac{\partial}{\partial r}\left(r^{2} \rho_{s} v_{s}^{2}\right)+2 \tau_{s} r^{2} \frac{1}{\rho_{s}} \frac{\partial p_{v}}{\partial r}\left(\frac{\partial p_{s}}{\partial r}-\frac{\partial p_{v}}{\partial r}\right) \\
-\tau_{s} \frac{\partial}{\partial r}\left(r^{2} \frac{\partial}{\partial r}\left(p_{s} v_{s}^{2}\right)\right)-5 \tau_{s} \frac{\partial}{\partial r}\left(r^{2} \frac{\partial}{\partial r}\left(\frac{p_{s}^{2}}{\rho_{s}}\right)\right)=0 .
\end{gathered}
$$

For the PV description we use

$$
\begin{gathered}
R_{v}=\frac{\partial p_{v}}{\partial r} \\
\frac{\partial}{\partial r}\left[3 p_{v} \frac{\partial v_{v}}{\partial r}+v_{v} \frac{\partial p_{v}}{\partial r}+\frac{4}{r} p_{v} v_{v}\right]-\frac{2}{r}\left[v_{v} \frac{\partial p_{v}}{\partial r}-p_{v} \frac{\partial v_{v}}{\partial r}\right]=0,
\end{gathered}
$$




$$
\begin{aligned}
& 5 p_{v} \frac{\partial v_{v}}{\partial r}+3 v_{v} \frac{\partial p_{v}}{\partial r}+\frac{10}{r} p_{v} v_{v}-\tau_{v} \frac{\partial}{\partial r}\left[16 p_{v} v_{v} \frac{\partial v_{v}}{\partial r}+5 v_{v}^{2} \frac{\partial p_{v}}{\partial r}\right]-\frac{14}{r^{2}} \tau_{v} p_{v} v_{v}^{2} \\
& -20 \frac{1}{r} \tau_{v} v_{v}^{2} \frac{\partial p_{v}}{\partial r}-\frac{60}{r} \tau_{v} p_{v} v_{v} \frac{\partial v_{v}}{\partial r}+4 \tau_{v} v_{v} \frac{\partial p_{v}}{\partial r} \frac{\partial v_{v}}{\partial r}-\frac{5}{r^{2}} \frac{\partial}{\partial r}\left[\tau_{s} r^{2} \frac{\partial}{\partial r} \frac{p_{s}^{2}}{\rho_{s}}\right]=0
\end{aligned}
$$

We use the system of equation written in the dimensionless form using the scales

$$
\left[r_{0}\right],\left[u_{0}\right],\left[p_{0}\right],[F]=\frac{p_{0}}{r_{0}},\left[t_{0}\right]=\frac{r_{0}}{u_{0}}, r_{0}=u_{0} t_{0} .
$$

In particular

$$
\begin{gathered}
\tilde{R}_{v}=\frac{\partial \tilde{p}_{v}}{\partial \tilde{r}} \\
\frac{\partial}{\partial \tilde{r}}\left[3 \tilde{p}_{v} \frac{\partial \tilde{v}_{v}}{\partial \tilde{r}}+\tilde{v}_{v} \frac{\partial \tilde{p}_{v}}{\partial \tilde{r}}+\frac{4}{\tilde{r}} \tilde{p}_{v} \tilde{v}_{v}\right]-\frac{2}{\tilde{r}}\left[\tilde{v}_{v} \frac{\partial \tilde{p}_{v}}{\partial \tilde{r}}-\tilde{p}_{v} \frac{\partial \tilde{v}_{v}}{\partial \tilde{r}}\right]=0,
\end{gathered}
$$

In this case system of Equations (10.7)-(10.12) written in the dimensionless form contains the dimensionless parameter, $\mathrm{T} \leftrightarrow \tilde{\tau}$ and need ten Cauchy conditions. These conditions we write down for the external surface of the spherical object. Then we investigate the evolution of the surface perturbation on the following scenario of the PV + Matter behavior.

We take into account the possible variations of the PV nonlocal parameter $\tau_{v}$. In this case we should introduce two nonlocal parameters for Matter $\tau_{s}$ and PV $\tau_{v}$. I underline again that nonlocal parameters in nonlocal physics play the same role as kinetic coefficients in usual local Boltzmann kinetic theory.

The Maple program was used in calculations $\left(\tau_{v} \neq 0\right)$ including the energy income. In this case we need the values without taking into account the influence of PV. The Maple notations were used:

$v_{s} \rightarrow \mathrm{u}-$ matter velocity with $\mathrm{PV}, \mathrm{v}-$ matter velocity without $\mathrm{PV}$;

$p_{s} \rightarrow \mathrm{p}$-matter pressure with $\mathrm{PV}, \mathrm{w}$-matter pressure without $\mathrm{PV}$;

$\rho_{s} \rightarrow \mathrm{r}-$ matter density with $\mathrm{PV}, \mathrm{m}$-matter density without PV.

For the surrounding PV motion we use:

$v_{v} \rightarrow \mathrm{s}-\mathrm{PV}$ velocity, $\quad p_{v} \rightarrow \mathrm{q}-\mathrm{PV}$ pressure, $\tilde{r} \rightarrow \mathrm{t}$, nonlocal parameter for Matter $\tilde{\tau}_{s} \rightarrow \mathrm{T}$, nonlocal parameter for $\mathrm{PV} \tilde{\tau}_{v} \rightarrow \mathrm{A}$. Let us introduce the energy income from PV to Matter defined as

$$
a(t)=\left(w(t)+m(t) * v(t)^{2}\right)-\left(p(t)+r(t) * u(t)^{2}\right) .
$$

In the calculations we should use parameters T, A and of course Cauchy conditions which could be found in the program text.

$$
\begin{gathered}
\mathrm{w}(1)=1, \mathrm{v}(1)=1, \mathrm{~m}(1)=1, \mathrm{D}(\mathrm{w})(1)=1, \mathrm{D}(\mathrm{v})(1)=1, \mathrm{D}(\mathrm{m})(1)=1, \mathrm{a}(1)=0, \\
\mathrm{p}(1)=1, \mathrm{D}(\mathrm{p})(1)=1, \mathrm{q}(1)=1, \mathrm{D}(\mathrm{q})(1)=1, \mathrm{r}(1)=1, \mathrm{D}(\mathrm{r})(1)=0, \mathrm{u}(1)=1, \\
\mathrm{D}(\mathrm{u})(1)=0, \mathrm{~s}(1)=1, \mathrm{D}(\mathrm{s})(1)=1 .
\end{gathered}
$$

The Figures 19-34 reflects the corresponding calculations including the rate of income $\frac{\partial \tilde{a}}{\partial \tilde{r}}$. Figures contain the boundary of the solution existing. 


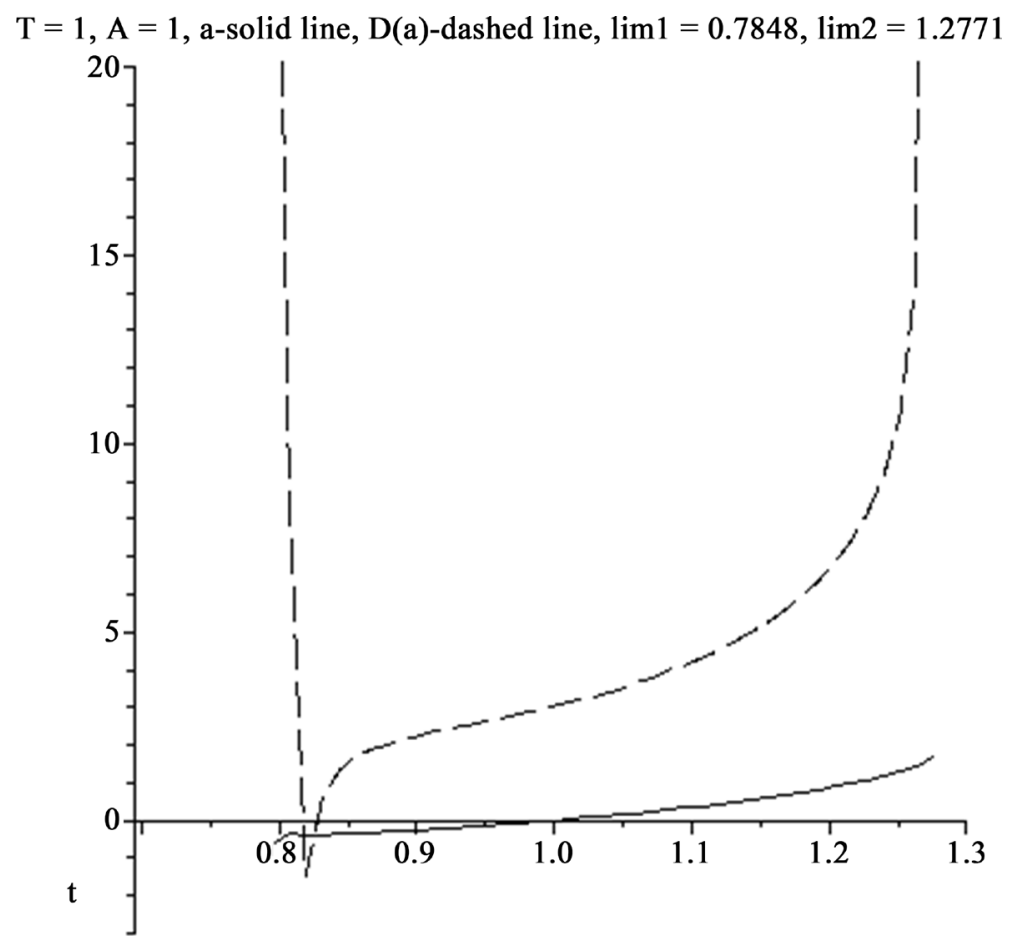

Figure 19. Energy income $\tilde{a}(\tilde{r})$, the rate of the energy income $\frac{\partial \tilde{a}}{\partial \tilde{r}}, \mathrm{~T}=\mathrm{A}=1$.

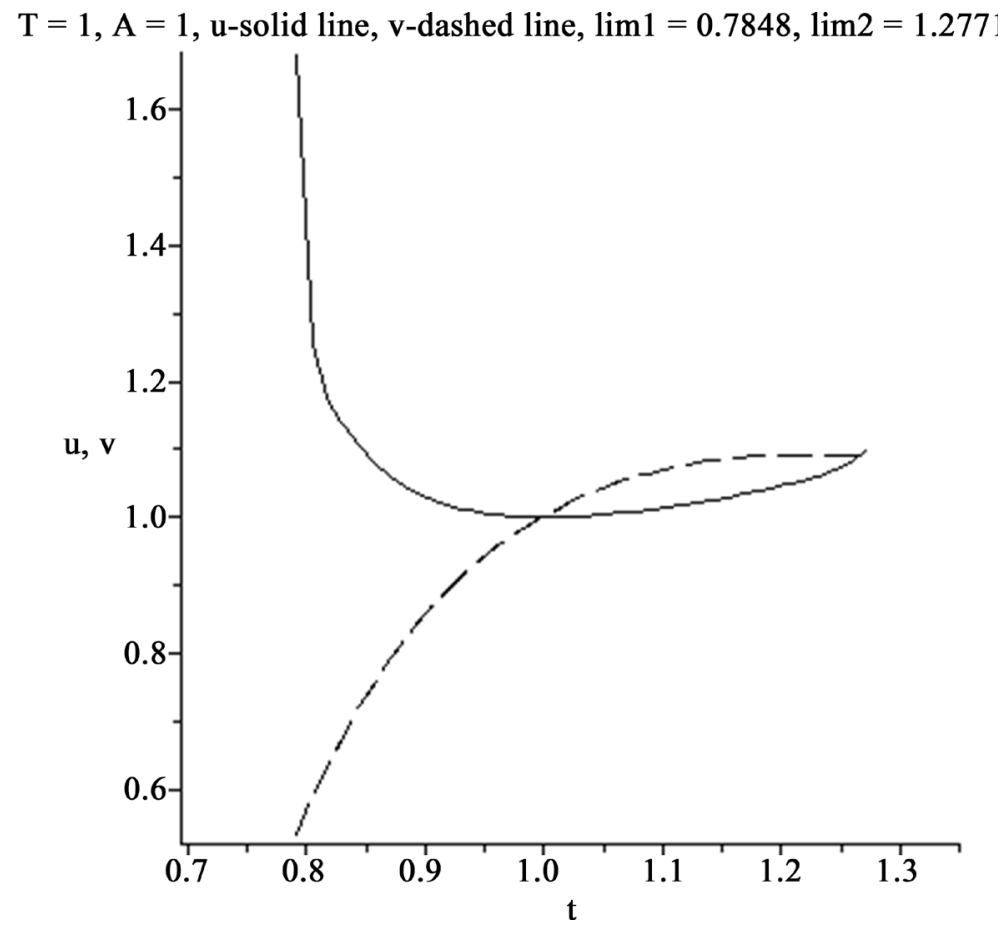

Figure 20. Evolution of matter velocity $\tilde{v}_{s}(\tilde{r}) \quad(\mathrm{u}(\mathrm{t})$ - with taking into account $\mathrm{PV}, \mathrm{v}(\mathrm{t}))$ - without PV influence), $\tilde{\tau}=1, \mathrm{~A}=1$. 
$\mathrm{T}=1, \mathrm{~A}=1, \mathrm{p}$-solid line, $\mathrm{w}$-dashed line, $\lim 1=0.7848, \lim 2=1.2771$

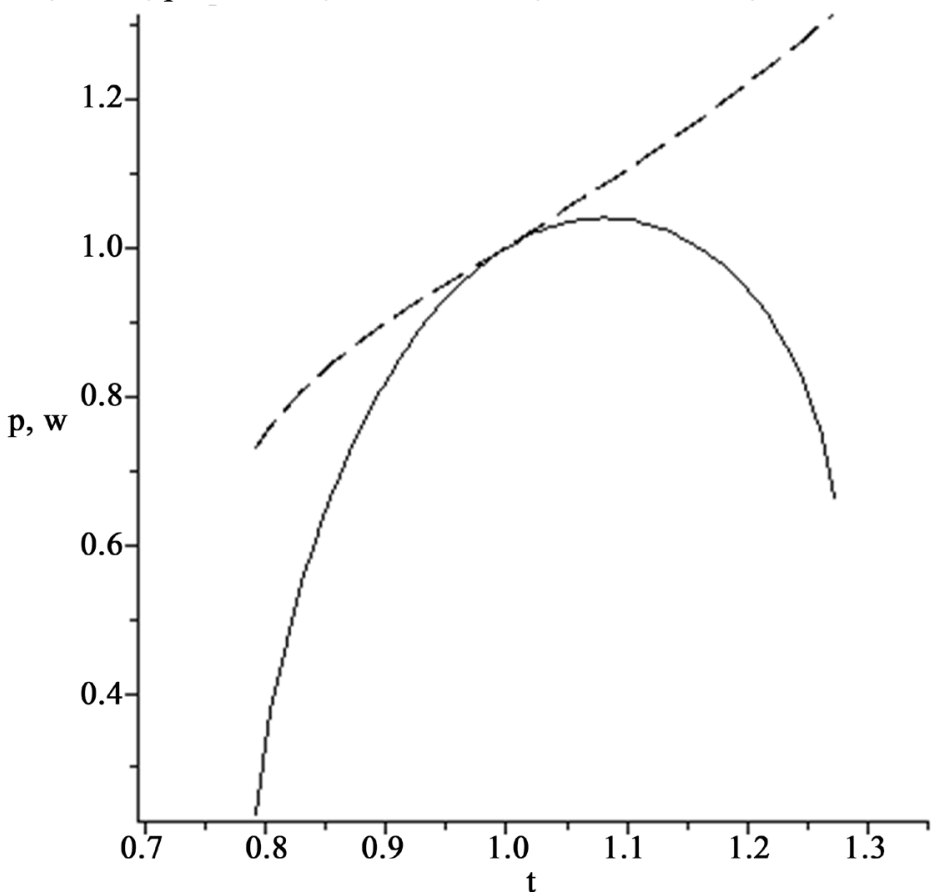

Figure 21. Evolution of matter pressure $\tilde{p}_{s}(\tilde{r})(\mathrm{p}(\mathrm{t})$ with PV influence, $\mathrm{w}(\mathrm{t})$ without PV influence), $\tilde{\tau}=1, \mathrm{~A}=1$.

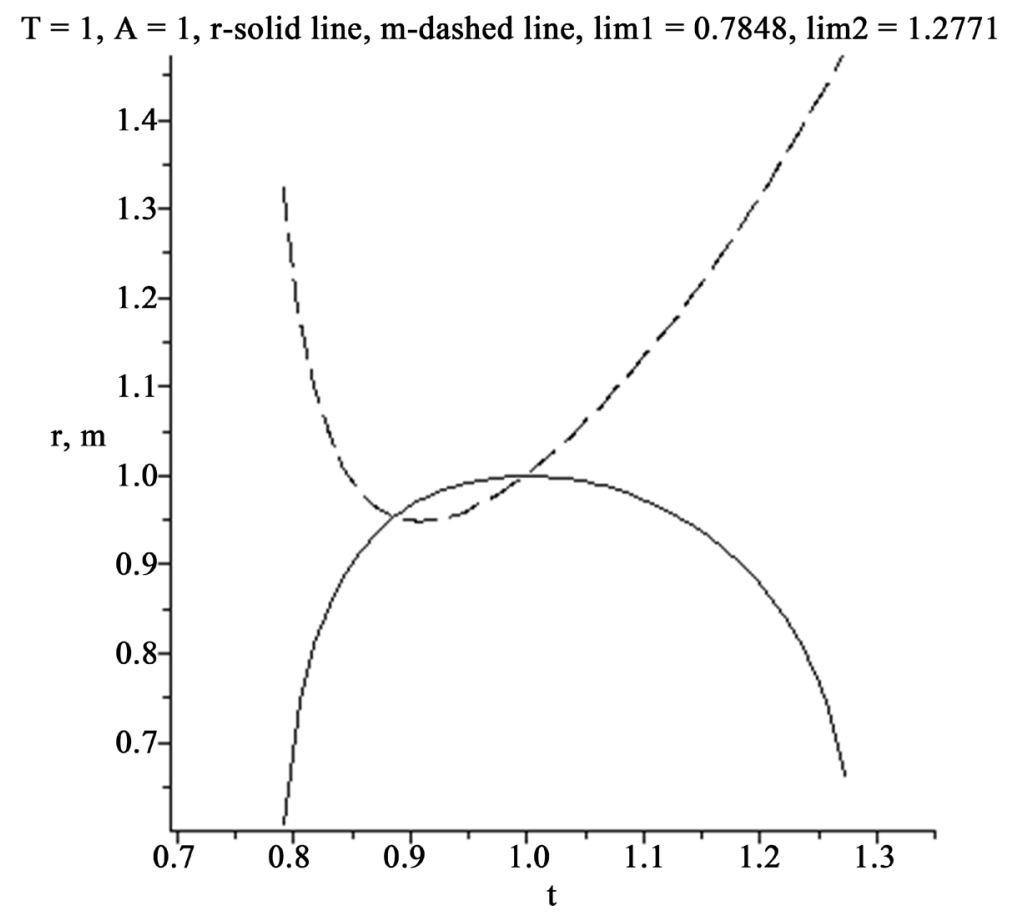

Figure 22. Evolution of matter density $\tilde{\rho}_{s}(\tilde{r})(\mathrm{r}(\mathrm{t})$ - with $\mathrm{PV}$ influence, $\mathrm{m}(\mathrm{t}))$ without $\mathrm{PV}$ influence), $\tilde{\tau}=1, \mathrm{~A}=1$. 


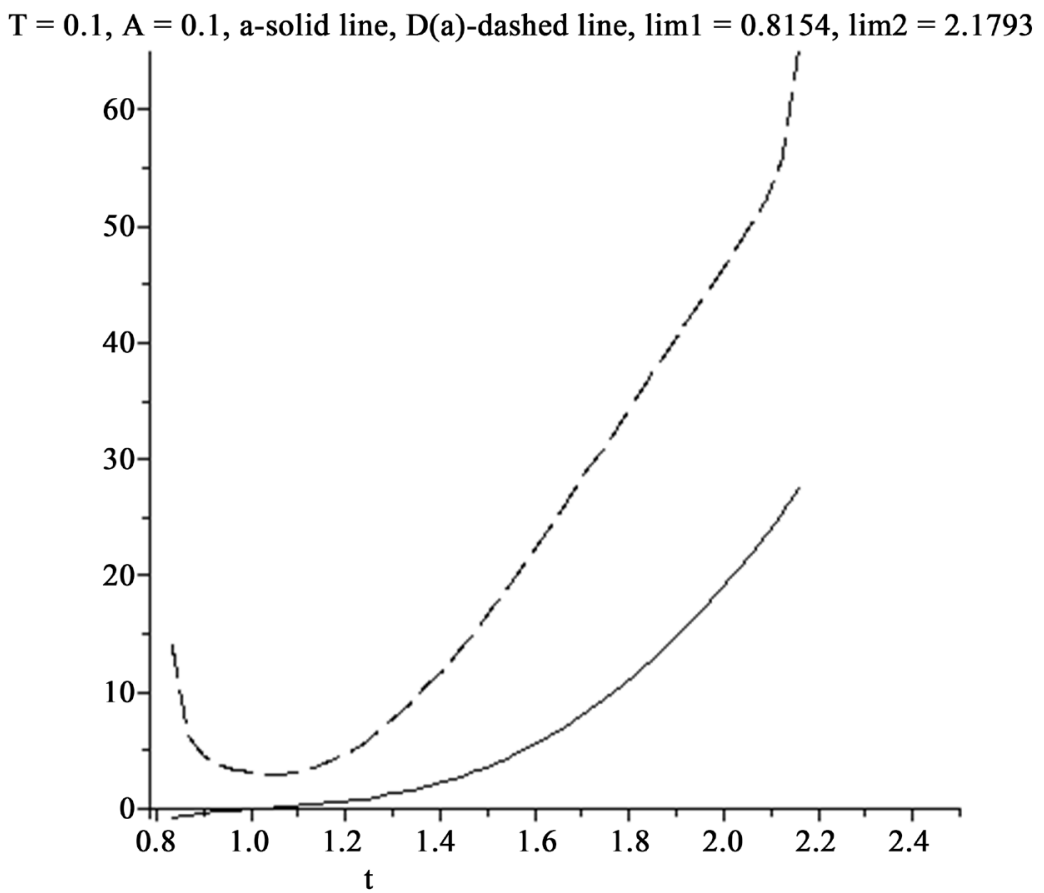

Figure 23. Energy income $\tilde{a}(\tilde{r})$, the rate of the energy income $\frac{\partial \tilde{a}}{\partial \tilde{r}}, \mathrm{~T}=\mathrm{A}=0.1$.

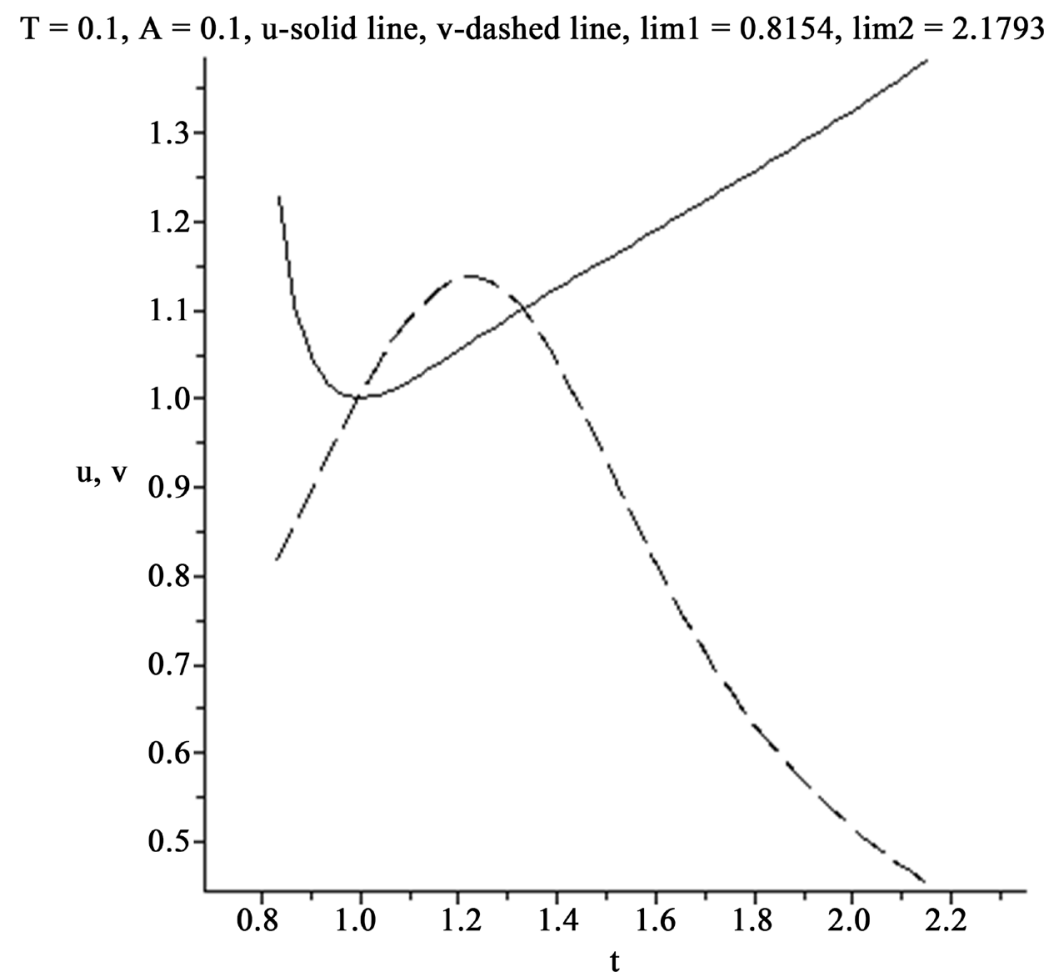

Figure 24. Evolution of matter velocity $\tilde{v}_{s}(\tilde{r}) \quad(\mathrm{u}(\mathrm{t})$ with $\mathrm{PV}$ influence, $\mathrm{v}(\mathrm{t})$ without $\mathrm{PV}$ influence), $\tilde{\tau}=0.1, \mathrm{~A}=0.1$. 


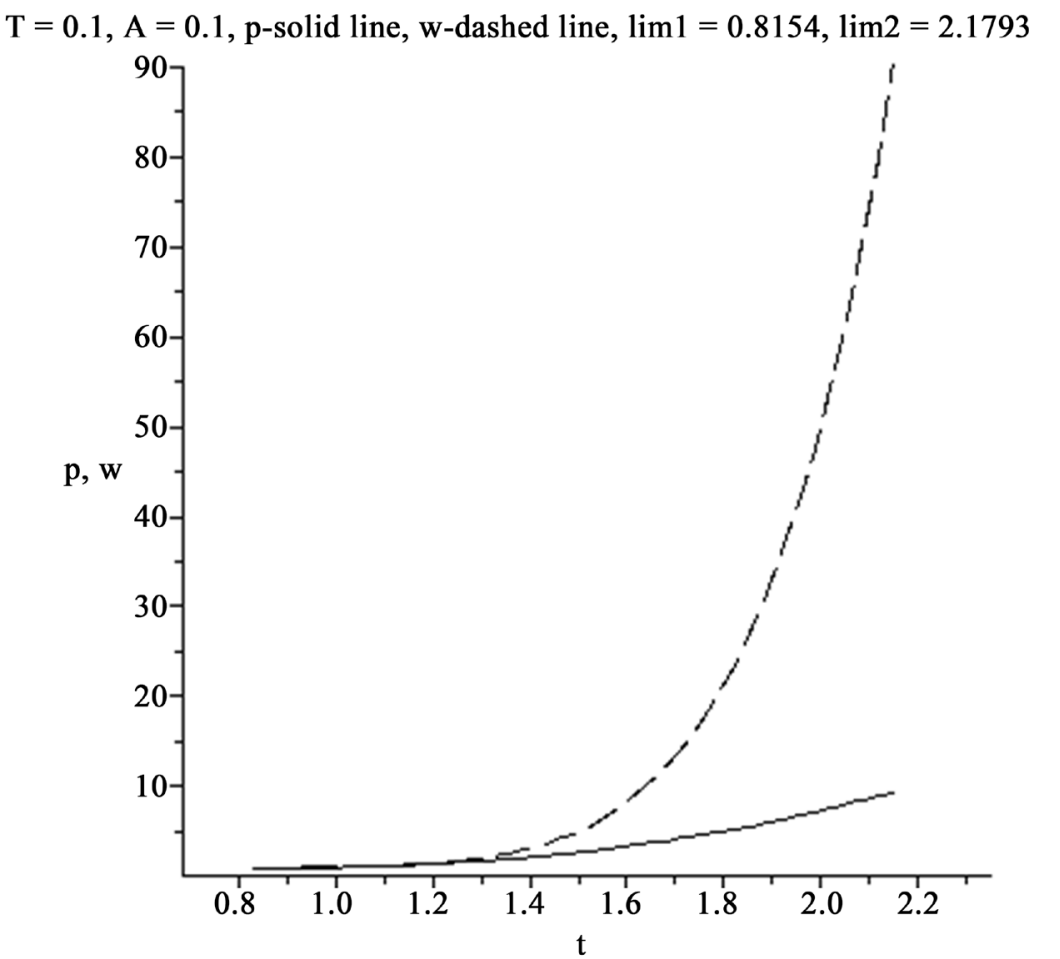

Figure 25. Evolution of matter pressure $\tilde{p}_{s}(\tilde{r}) \quad(\mathrm{p}(\mathrm{t})$ - with PV influence, $\mathrm{w}(\mathrm{t})$ - without PV influence, $\tilde{\tau}=0.1, \mathrm{~A}=0.1$.

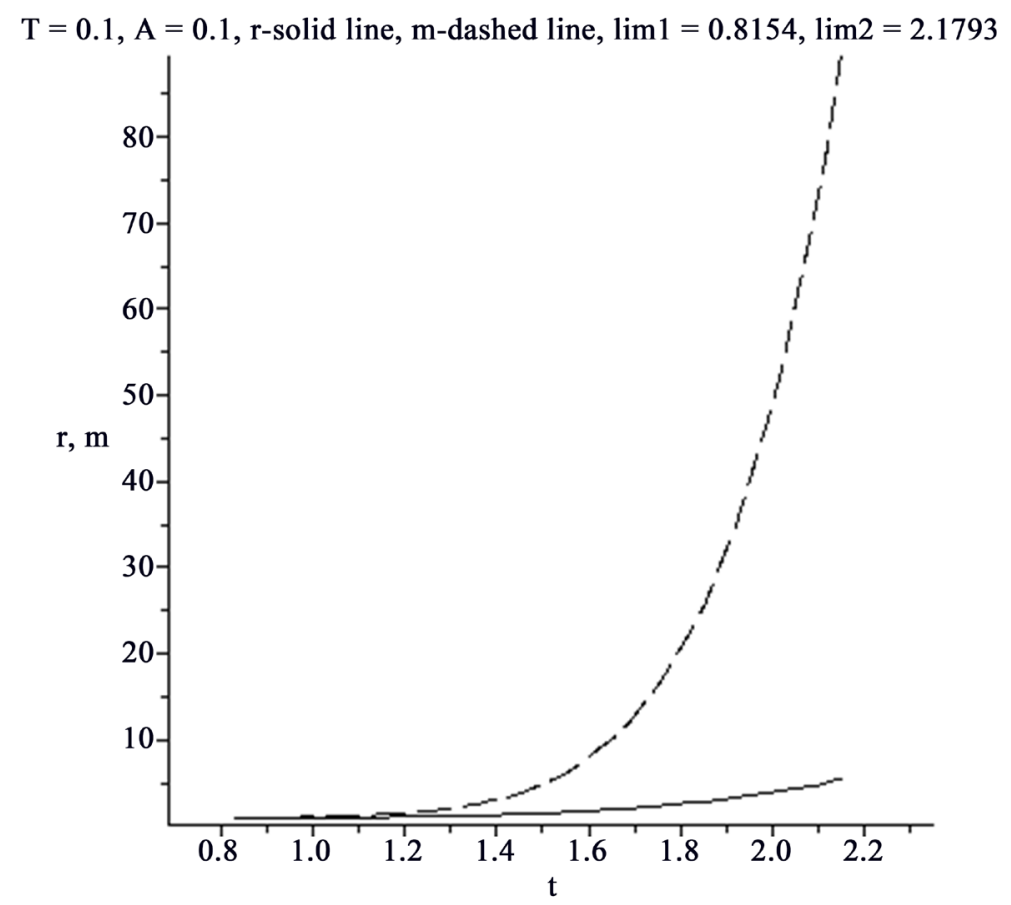

Figure 26. Evolution of matter density $\tilde{\rho}_{s}(\tilde{r}) \quad(\mathrm{r}(\mathrm{t})$ with PV influence, $\mathrm{m}(\mathrm{t}))$ without PV influence), $\tilde{\tau}=0.1, \mathrm{~A}=0.1$. 
$\mathrm{T}=0.1, \mathrm{~A}=0.01$, a-solid line, $\mathrm{D}(\mathrm{a})$-dashed line, $\lim 1=0.8486, \lim 2=2.0702$

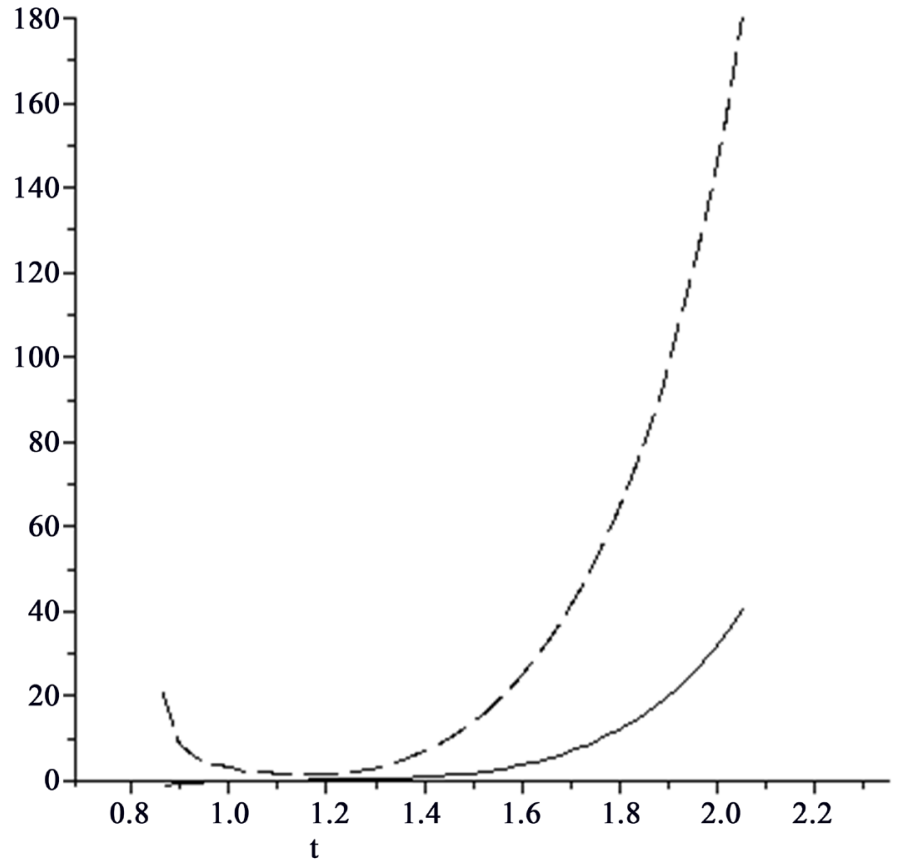

Figure 27. Energy income $\tilde{a}(\tilde{r})$, the rate of the energy income $\frac{\partial \tilde{a}}{\partial \tilde{r}}, T=0.1, A=0.01$.

$\mathrm{T}=0.1, \mathrm{~A}=0.01, \mathrm{u}$-solid line, $\mathrm{v}$-dashed line, $\lim 1=0.8486, \lim 2=2.0702$

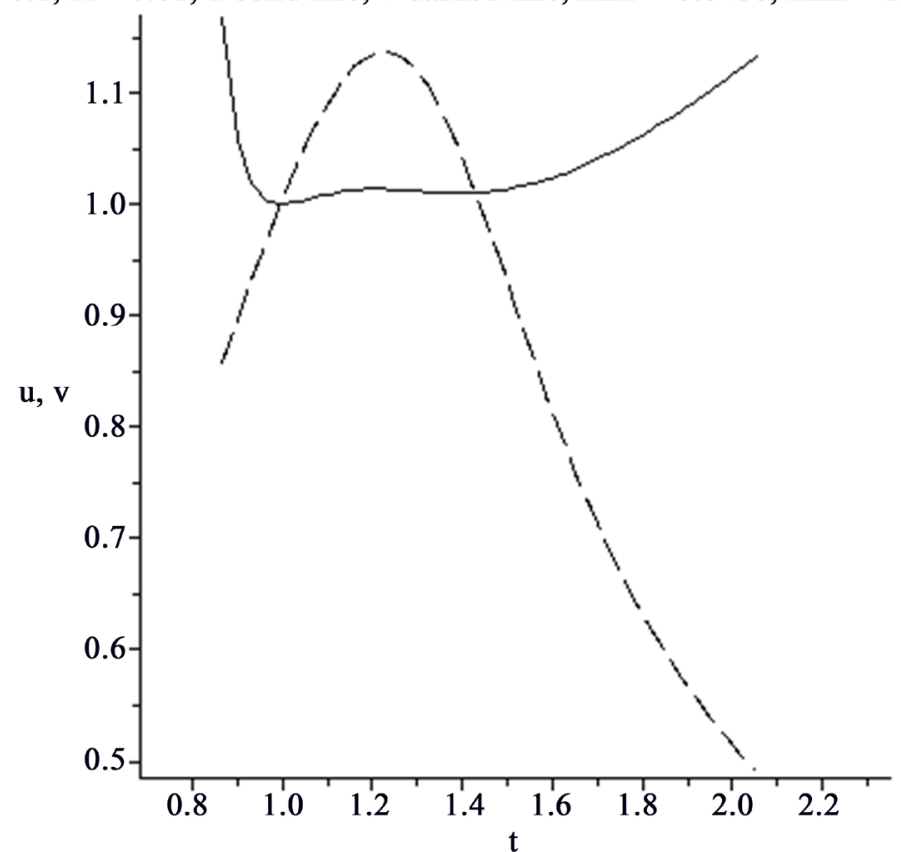

Figure 28. Evolution of matter velocity $\tilde{v}_{s}(\tilde{r})(\mathrm{u}(\mathrm{t})$ - with PV influence, $\mathrm{v}(\mathrm{t})$ - without PV influence), $\tilde{\tau}=0.1, \mathrm{~A}=0.01$. 


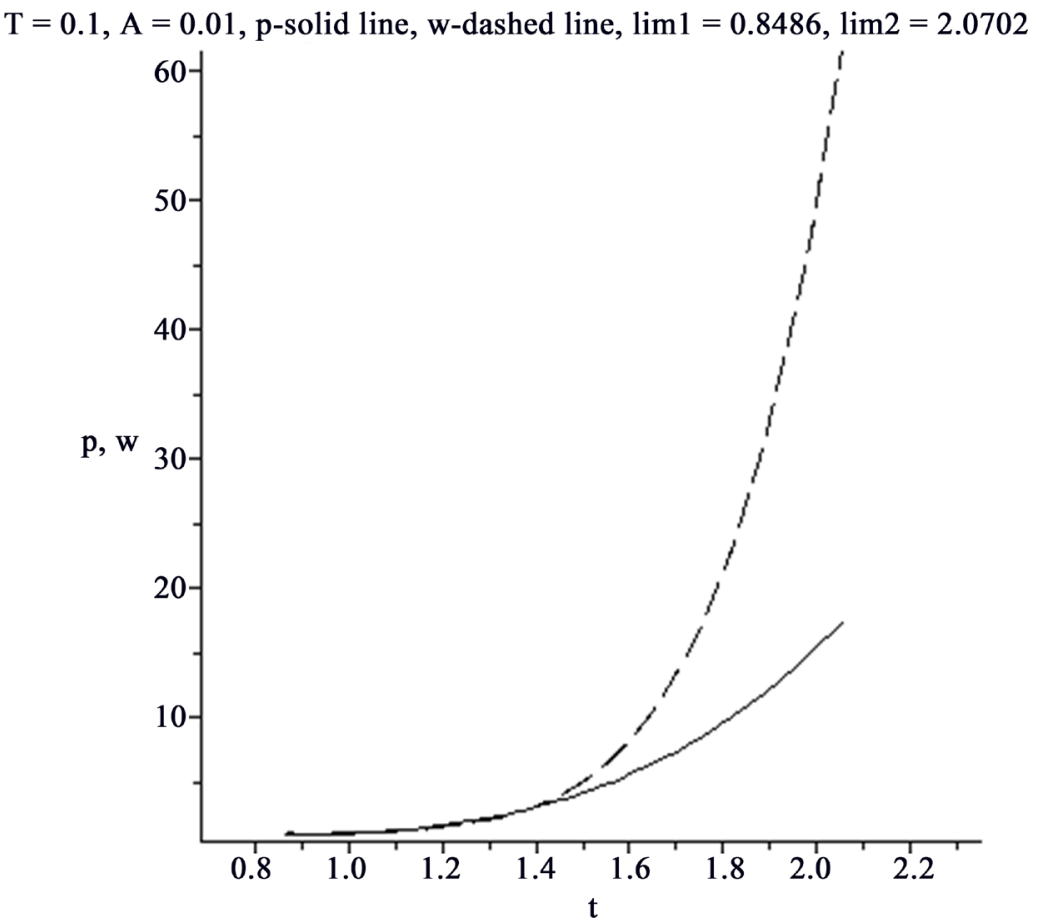

Figure 29. Evolution of matter pressure $\tilde{p}_{s}(\tilde{r})(\mathrm{p}(\mathrm{t}))$ - with PV influence, $\mathrm{w}(\mathrm{t})$ - without PV influence), $\tilde{\tau}=0.1, \mathrm{~A}=0.01$.

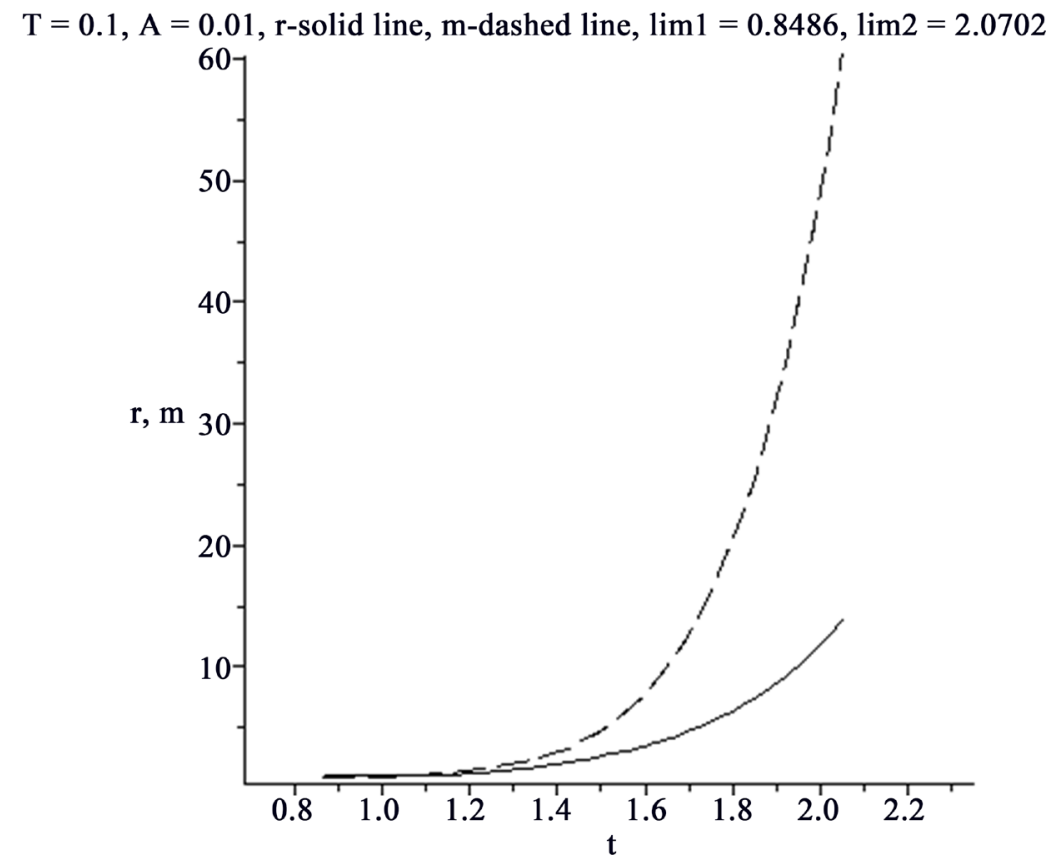

Figure 30. Evolution of matter density $\tilde{\rho}_{s}(\tilde{r})(\mathrm{r}(\mathrm{t})$ - with $\mathrm{PV}$ influence, $\mathrm{m}(\mathrm{t})$ - without PV influence), $\tilde{\tau}=0.1, \mathrm{~A}=0.1$. 


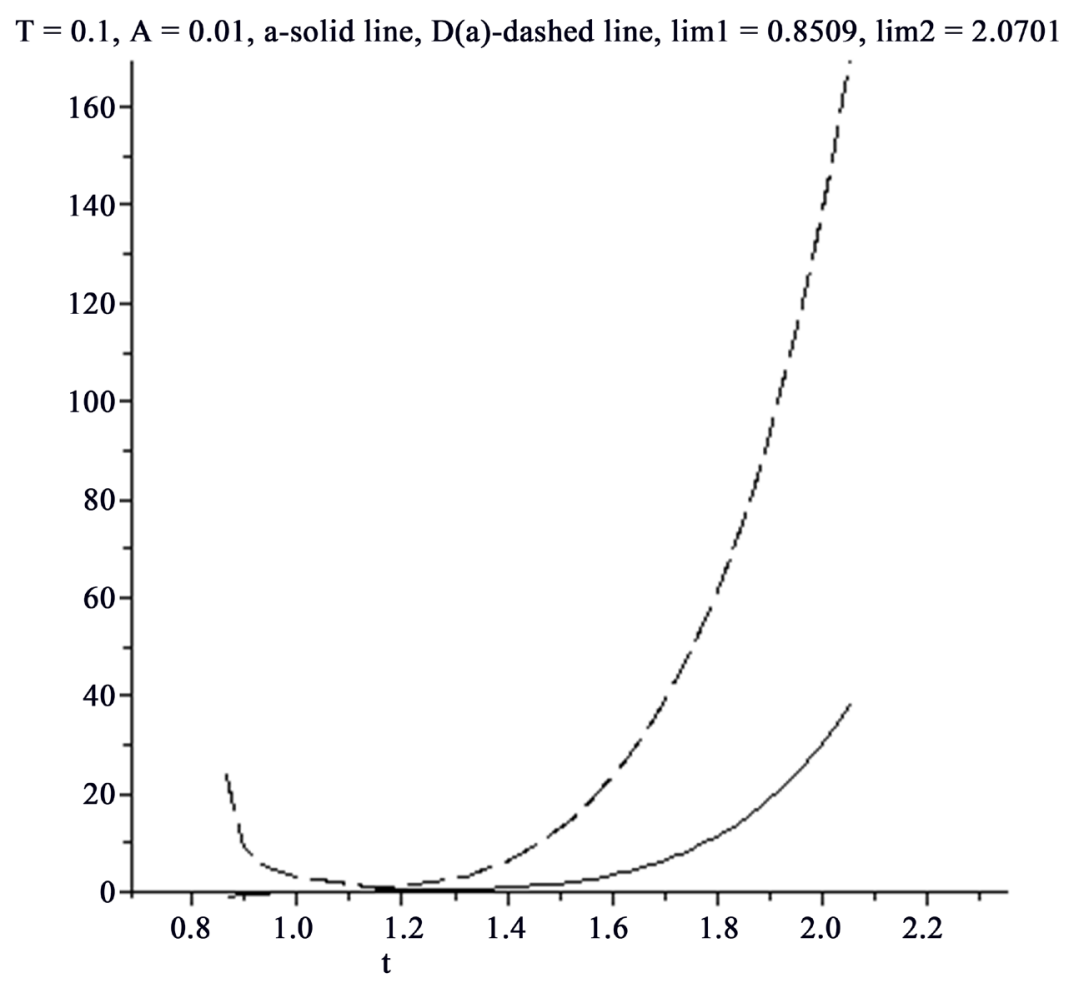

Figure 31. Energy income $\tilde{a}(\tilde{r})$, the rate of the energy income $\frac{\partial \tilde{a}}{\partial \tilde{r}}, \mathrm{~T}=0.1$, $\mathrm{A}=0.001$.

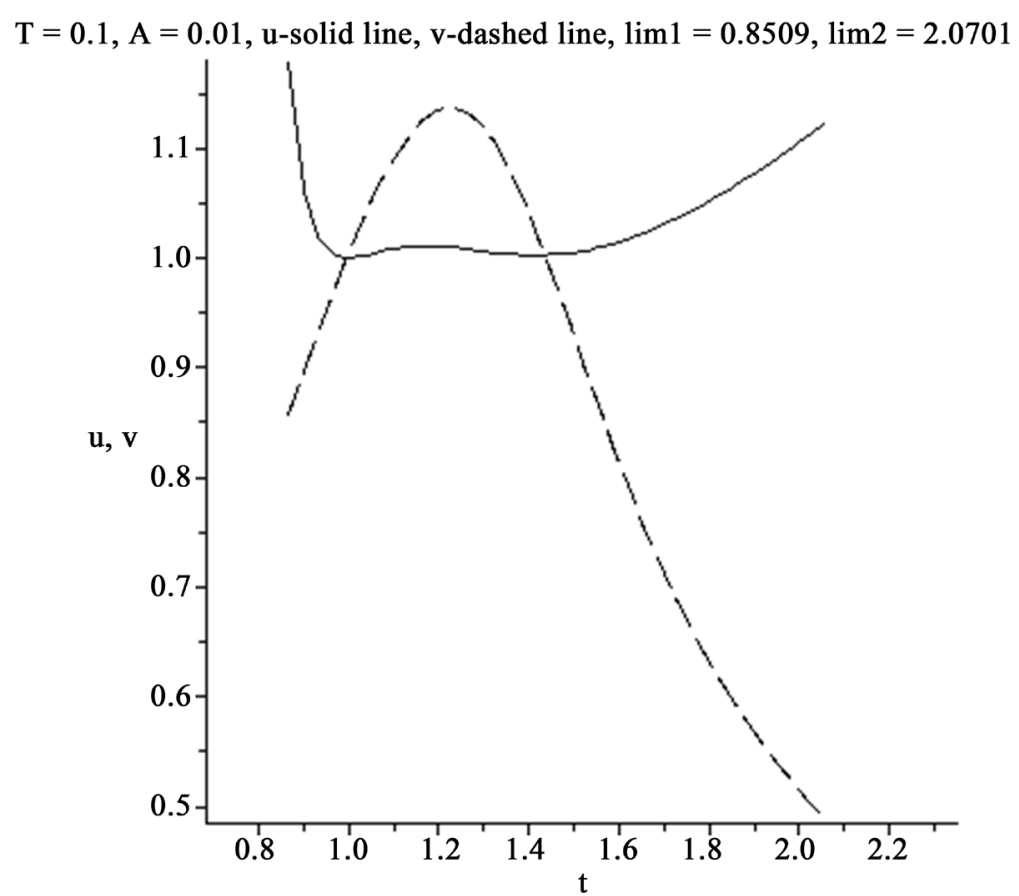

Figure 32. Evolution of matter velocity $\tilde{v}_{s}(\tilde{r})$ ( $\mathrm{u}(\mathrm{t})$ with $\mathrm{PV}$ influence, $\mathrm{v}(\mathrm{t})$ without $\mathrm{PV}$ influence), $\tilde{\tau}=0.1, \mathrm{~A}=0.001$. 
$\mathrm{T}=0.1, \mathrm{~A}=0.01, \mathrm{p}$-solid line, $\mathrm{w}$-dashed line, $\lim 1=0.8509, \lim 2=2.0701$

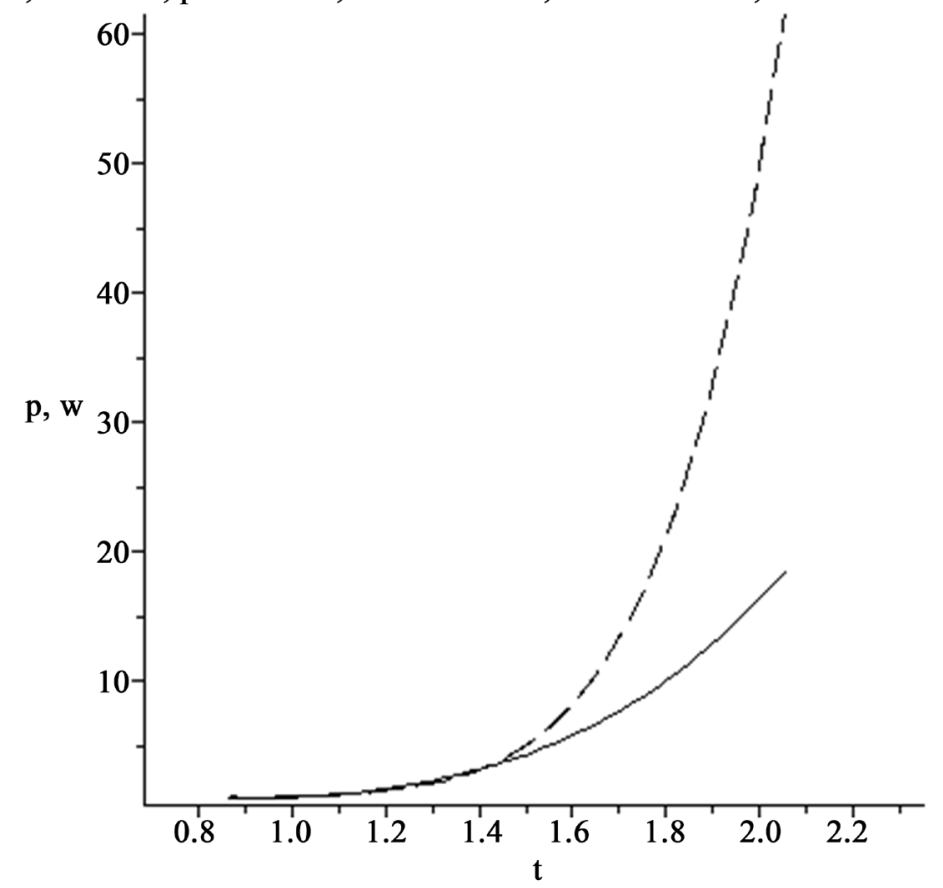

Figure 33. Evolution of matter pressure $\tilde{p}_{s}(\tilde{r})(\mathrm{p}(\mathrm{t})$ with $\mathrm{PV}$ influence, $\mathrm{w}(\mathrm{t})$ without PV influence), $\tilde{\tau}=0.1, \mathrm{~A}=0.001$.

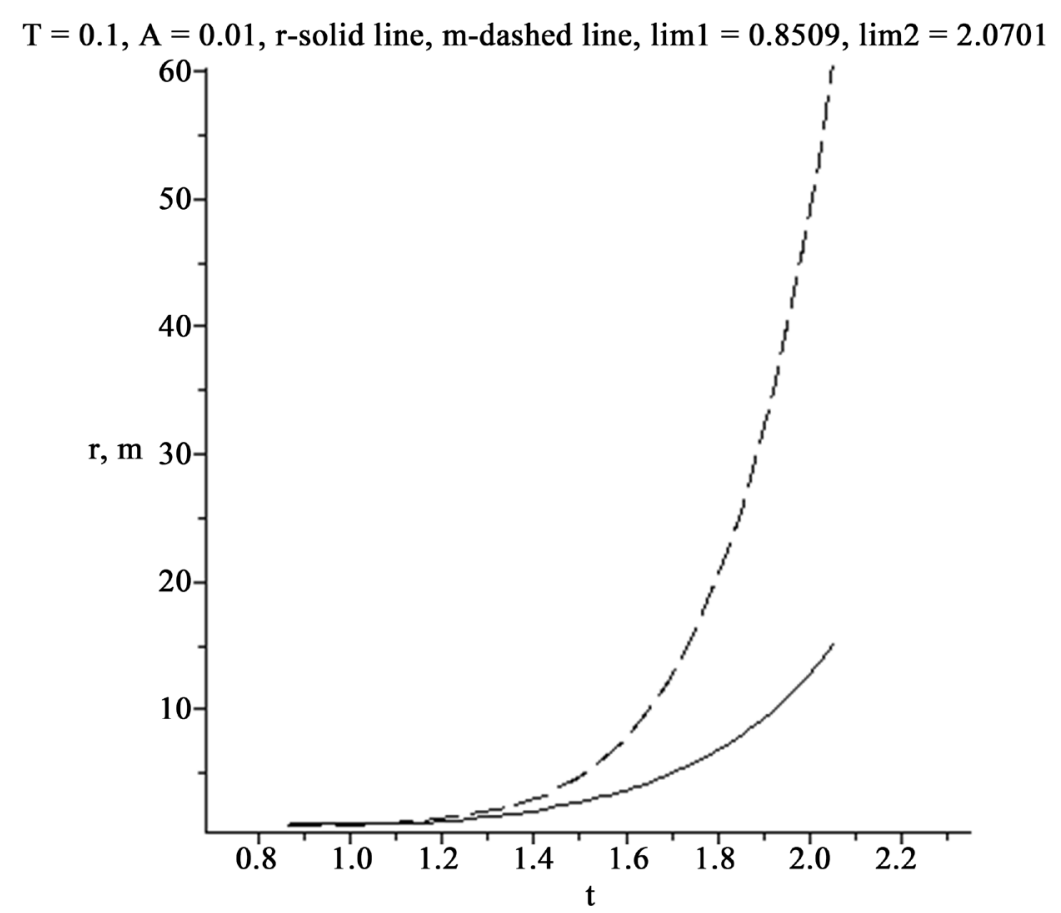

Figure 34. Evolution of matter density $\tilde{\rho}_{s}(\tilde{r})(\mathrm{r}(\mathrm{t})$ with $\mathrm{PV}$ influence, $\mathrm{m}(\mathrm{t})$ without $\mathrm{PV}$ influence, $\tilde{\tau}=0.1, \mathrm{~A}=0.001$. 


\section{Conclusion to Item 10:}

1) In many cases mentioned calculations lead to so to speak "volume quantization"-the solutions exist only in the finite domain of space, (see for example Figures 19-34).

2) The left boundary of the solution existing can be smaller than the sphere boundary $\tilde{r}=1$.

3) The linear size of these domains demonstrate the weak dependence on nonlocal parameters $\tilde{\tau}_{s}$ and $\tilde{\tau}_{v}$. From the other side the parameters of the energy income show the strong dependence on nonlocal parameters. The nonlocal theory can show the explosion of object which size is significantly less than the size of the visible Universe.

4) Extremely important that the direct calculations demonstrate the possibility of the energy income from PV to Matter.

\section{Final Remarks: The Destiny of Anti-Matter after Big Bang (BB) and Conclusion}

The problem antimatter evolution is considered from positions of the Newtonian theory of gravitation and non-local kinetic theory. It is found that disappearing of antimatter after Big Bang can be explained as a result of antimatter interaction with physical vacuum. This interaction leads to appearance of the antimatter particles which cross-sections are significantly less than the crosssections of the ordinary matter. As a result the main part of antimatter is concentrated now on the out part of the visible Universe.

Application nonlocal physics to the problem of the dark matter existence leads to affirmation - dark matter does not exist.

Physical Vacuum (PV) is not a speculative object; it is a reality as "matter" and "fields". In other words, the physical vacuum is "the third" physical reality along with matter and fields. At an early stage in the development of the universe the value of the PV parameter is closer to zero. As a result the antimatter particles having the small cross sections, leaves the central part of the BB domain. Anti-matter particles after the Big Bang are placed now mainly on the periphery of the Universe. We observe now the effects of the matter antimatter annihilations on the periphery of our Universe.

The birth of the universe is convoying of appearance of the repulsion forces. In the existing terminology we discover the "negative pressure" and "dark energy" in all cases. This fundamental result does not depend on the mechanism of external perturbations. In other words, the anti-gravity in the physical vacuum exists, if there is dissipation of energy or in the absence of dissipation at all.

We find the solutions of the transport equations defining the evolution the physical vacuum (PV).

It means:

1) If the matter is absent, non-local evolution equations have nevertheless non-trivial solutions corresponding evolution of PV which description in time 
and 3D space on the level of quantum hydrodynamics demands only quantum pressure $p$, the self-consistent force $\mathbf{F}$ (acting on unit of the space volume) and velocity $\mathbf{v}_{0}$. The system of non local equations is written for the case when the usual matter is absent $(\rho=0)$, also radiation, gravitation (as well as other mass forces) and electromagnetic fields are absent.

2) In all other cases we consider from the position of the nonlocal physics the interaction of Physical Vacuum with the external electromagnetic and gravitational fields taking into account the possible technical applications like EM-engine.

3) The general relativity (GR) and the special relativity (SR) are not applicable for the description of processes in Physical Vacuum.

4) An image of the entire universe consists mostly of colossal collections of galaxies interspersed with vast empty spaces, known as voids. The appearance of void is usually associated with Big Bang and appearance of the Cold Spot. Correlation between CMB fluctuations, voids and clusters (Kovács et al. 2017) was found including Cold Spot related to Eridanus Supervoid (Szapudi et al, 2015). But there many observed voids. Cosmic voids are vast spaces which contain very few or no galaxies. Voids typically have a diameter of 10 to more than 100 megaparsecs. The existence of limited spherical formations in the Universe filled with interacting matter and physical vacuum is the justification for the existence of voids and parallel universes.

5) It means that we should wait for the tremendous discoveries in the nonlocal theory of voids. In particular, some voids may have been formed by their own local PV explosions (like Big Bang). But the result of such local PV explosions could lead to the appearance of so-called "parallel universes". These post-explosion universes may have had a different set of fundamental physical constants and therefore a different flow of time. Then we should search the voids with abnormal transport processes (from the point of view of the Earth science).

6) Direct calculations demonstrate the possibility of the energy income from PV to Matter.

\section{Acknowledgements}

Author is deeply indebted to Academicians A P Alexandrov, A A Dorodnitsin, V L Ginzburg, A A Samarskii, L I Sedov and Prof. F Uhlig for their interest in this work and in the subject in general.

\section{Conflicts of Interest}

The author declares no conflicts of interest regarding the publication of this paper.

\section{References}

[1] Boltzmann, L. (1872) Sitz. Ber. Kaiserl. Akad. Wiss, 66, No. 2, s.275.

[2] Alekseev, B.V. (1982) Matematicheskaya Kinetika Reagiruyushchikh Gazov (Ma- 
thematical Theory of Reacting Gases). Nauka, Moscow.

[3] Alexeev, B.V. (2004) Generalized Boltzmann Physical Kinetics. Elsevier, Amsterdam, 368 p. https://doi.org/10.1016/B978-044451582-7/50027-8

[4] Alexeev, B.V. (2015) Unified Non-Local Theory of Transport Processes. Elsevier, Amsterdam, $644 \mathrm{p}$.

[5] Alexeev, B.V. (2016) Unified Non-Local Relativistic Theory of Transport Processes. Elsevier, Amsterdam, $455 \mathrm{p}$.

[6] Alexeev, B.V. (2017) Nonlocal Astrophysics. Dark Matter, Dark Energy, Physical Vacuum. Elsevier, Amsterdam, $454 \mathrm{p}$.

[7] Alexeev, B.V. (1994) Philosophical Transactions of the Royal Society of London, 349, 417-443. https://doi.org/10.1098/rsta.1994.0140

[8] Alexeev, B.V. (1995) Physica A, 216, 459-468. https://doi.org/10.1016/0378-4371(95)00044-8

[9] Madelung, E. (1927) Zeitschrift für Physic, 40, 322-326. https://doi.org/10.1007/BF01400372

[10] Bell, J.S. (1964) Physics, 1, 195. https://doi.org/10.1103/PhysicsPhysiqueFizika.1.195

[11] Alexeev, B.V. (2008) Journal of Nanoelectronics and Optoelectronics, 3, 143-158. https://doi.org/10.1166/jno.2008.207

[12] Alexeev, B.V. (2008) Journal of Nanoelectronics and Optoelectronics, 3, 316-328. https://doi.org/10.1166/jno.2008.311

[13] Reinders, L.J. (2018) The Life, Science and Times of Lev Vasilevich Shubnikov. Springer International Publishing, Cham. https://doi.org/10.1007/978-3-319-72098-2

[14] Abrikosov, A.A. (1957) Journal of Experimental and Theoretical Physics, 5, 1174.

[15] Nikulov, A.V. (1999) The Vortex Lattice Melting Theory as Example of Science Fiction. In: Ausloos, M. and Kruchinin, S., Eds., NATO Science Series. Symmetry and Pairing in Superconductors, Kluwer Academic Publishers, Dordrecht, 131.

http://arXiv.org/abs/cond-mat/9811051 https://doi.org/10.1007/978-94-011-4834-4 12

[16] Nikulov, A.V. (1999) What Is the Vortex Lattice Melting, Reality or Fiction? In: Kossowski, R., et al., Eds., NATO Science Series: Physics and Materials Science of Vortex States, Flux Pinning and Dynamics, Kluwer Academic Publishers, Dordrecht, 609. http://arXiv.org/abs/physics/0202021 https://doi.org/10.1007/978-94-011-4558-9 31

[17] Kleiner, W.H., Roth, L.M. and Autler, S.H. (1964) Physical Review A, 133, 1226. https://doi.org/10.1103/PhysRev.133.A1226

[18] Maki, K and Takayama H. (1971) Progress of Theoretical Physics, 46, 1651-1665. https://doi.org/10.1143/PTP.46.1651

[19] Nikulov, A.V., Remisov, D.Yu. and Oboznov V.A. (1995) Physical Review Letters, 75, 2586. https://doi.org/10.1103/PhysRevLett.75.2586

[20] Nikulov, A.V., Dubonos, S.V. and Koval, Y.I. (1997) Journal of Low Temperature Physics, 109, 643-652. https://doi.org/10.1007/BF02435484

[21] Nikulov, A.V. (1995) Physical Review B, 52, 10429-10432. https://doi.org/10.1103/PhysRevB.52.10429

[22] Alexeev, B.V. (2021) Journal of Modern Physics, 12, 552-593.

https://www.scirp.org/journal/jmp https://doi.org/10.4236/jmp.2021.125037 
[23] Michelson, A.A. and Morley, E.W. (1887) American Journal of Science, 34, 333-345. https://doi.org/10.2475/ajs.s3-34.203.333

[24] Lorentz, H.A. (1901) Aether Theories and Aether Models (1901-1902). Lectures on Theoretical Physics Delivered at the University of Leyden, Volume 1. Edited by H. Bremecamp.

[25] Logunov, A.A., Loskutov, Yu.M. and Mestvirishvili, M.A. (1987) TMF, 73, 163-186. https://doi.org/10.1007/BF01017582

[26] Laplace, P.S. (1966) Mechanique Celeste. English Transl. Reprinted by Chelsea Publ., New York.

[27] Poincaré, J.H. (1983) About Science. Nauka, Moscow.

[28] Alexeev, B.V. (2018) Extremal States in Nonlocal Physics. Lambert Academic Publishing, Riga.

[29] Prigogine, I. (1962) Introduction to Thermodynamics of Irreversible Processes. 2nd Edition, Wiley Interscience, London.

[30] Nikolis, G. and Prigogine, I. (1977) Self-Organization in Nonequilibrium Systems. Wiley-Interscience Publication, Hoboken.

[31] Rubakov, V.A. (2019) Science and Life, 11, 46-50.

[32] Penrose, R. (2011) The Road to Reality: A Complete Guide to the Laws of the Universe. Knopf (US), New York, Bodley Head, London.

[33] Rubin, V. and Ford, W.K. (1970) Astrophysical Journal, 159, 379. https://doi.org/10.1086/150317

[34] Rubin, V., Thonnard, N. and Ford, W.K. (1980) Astrophysical Journal, 238, 471. https://doi.org/10.1086/158003

[35] Zwicky, F. (1933) Helvetica Physica Acta, 6, 110-127.

[36] Zwicky, F. (1937) Astrophysical Journal, 86, 217. https://doi.org/10.1086/143864 